\title{
1 Dynamic 3D chromosomal landscapes in acute leukemia
}

3 Andreas Kloetgen ${ }^{1,2, *}$, Palaniraja Thandapani ${ }^{1,2, *}$, Panagiotis Ntziachristos ${ }^{1,2,3,4, *}$, Yohana 4 Ghebrechristos $^{1,2}$, Sofia Nomikou ${ }^{1,2}$, Charalampos Lazaris ${ }^{1,2}$, Xufeng Chen ${ }^{1,2}$, Hai $\mathrm{Hu}^{1,2}$, Sofia 5 Bakogianni ${ }^{1,5}$, Jingjing Wang ${ }^{1,2}$, Yi Fu ${ }^{6}$, Francesco Boccalatte ${ }^{1,2}$, Hua Zhong ${ }^{7}$, Elisabeth Paietta ${ }^{8}$, 6 Thomas Trimarchi ${ }^{1,2}$, Yixing Zhu ${ }^{3}$, Pieter van Vlierberghe ${ }^{9}$, Giorgio G Inghirami ${ }^{10}$, Timothee 7 Lionnet $^{6}$, Iannis Aifantis ${ }^{1,2,12, \#}$ and Aristotelis Tsirigos ${ }^{1,2,11,12, \#}$

$9 \quad{ }^{1}$ Department of Pathology; New York University School of Medicine, New York, NY 10016, USA

$10 \quad{ }^{2}$ Laura \& Isaac Perlmutter Cancer Center, New York University School of Medicine, New York, NY 11 10016, USA

$12{ }^{3}$ Department of Biochemistry and Molecular Genetics, Feinberg School of Medicine, Northwestern 13 University, Chicago, IL, 60611, USA.

$14{ }^{4}$ Robert H. Lurie Comprehensive Cancer Center, Northwestern University, Chicago, IL, 60611, USA.

$15{ }^{5}$ Department of Microbiology, Alexandria Center for Life Sciences, New York University School of 16 Medicine, New York, NY, 10016, USA.

$17{ }^{6}$ Department of Cell Biology; Institute for Systems Genetics, New York University, New York, NY, 18 10016, USA.

$19{ }^{7}$ Division of Biostatistics, Department of Population Health, NYU School of Medicine, New York, NY, 20 USA.

$21 \quad{ }^{8}$ Montefiore Medical Center, 111 East 210th Street, New York, NY, USA.

$22{ }^{9}$ Department of Biomolecular Medicine, Ghent University, Ghent, Belgium.

$23{ }^{10}$ Department of Pathology and Laboratory Medicine, Weill Cornell Medical College, New York, NY, 24 USA.

$25{ }^{11}$ Applied Bioinformatics Laboratories, Office of Science \& Research, NYU School of Medicine, New 26 York, NY, 10016, USA.

$27 \quad *, 12$ These authors contributed equally to the work.

\#Correspondence to:

30 Aristotelis Tsirigos, Email: Aristotelis.Tsirigos@ nyulangone.org

31 Iannis Aifantis, Email: Ioannis.Aifantis@nyulangone.org 


\section{ABSTRACT}

2 Three-dimensional (3D) chromatin architectural changes can alter the integrity of topologically 3 associated domains (TADs) and rewire specific enhancer-promoter interactions impacting gene

4 expression. Recently, such alterations have been implicated in human disease, highlighting the 5 need for a deeper understanding of their role. Here, we investigate the reorganization of 6 chromatin architecture in T cell acute lymphoblastic leukemia (T-ALL) using primary human 7 leukemia specimens and its dynamic responses to pharmacological agents. Systematic 8 integration of matched in situ Hi-C, RNA-Seq and CTCF ChIP-Seq datasets revealed widespread 9 changes in intra-TAD chromatin interactions and TAD boundary insulation in T-ALL. Our 10 studies identify and focus on a TAD "fusion" event being associated with loss of CTCF11 mediated insulation, enabling direct interactions between the MYC promoter and a distal super12 enhancer. Moreover, our data show that small molecule inhibitors targeting either oncogenic 13 signal transduction or epigenetic regulation reduce specific 3D interactions associated with 14 transformation. Overall, our study highlights the impact, complexity and dynamic nature of 3D 15 chromatin architecture in human acute leukemia.

17 Keywords: Chromatin conformation capture (3C), T-cell acute lymphoblastic leukemia (T18 ALL), Topologically Associated Domains (TADs), gamma-secretase inhibition ( $\gamma \mathrm{SI}$ ), Cyclin19 dependent kinase 7 (CDK7) inhibition.

21 One Sentence Summary: 3D chromatin alterations in T cell leukemia are accompanied by 22 changes in insulation and oncogene expression and can be partially restored by targeted drug 23 treatments. 


\section{INTRODUCTION}

2 The human genome is replete with regulatory elements such as promoters, enhancers and

3 insulators. Recent findings have highlighted the impact of the spatial genome organization in

4 governing the physical proximity of these elements for the precise control of gene expression ${ }^{1-3}$.

5 Genome organization is a multistep process that involves compacting chromatin into

6 nucleosomes, chromatin fibers, compartments and into chromosome territories ${ }^{3,4}$. Multiple lines

7 of evidence suggest that at the sub-megabase level, the genome is organized in distinct, non-

8 overlapping regions of highly self-interacting chromatin, called topologically associated domains

9 (TADs) ${ }^{5-7}$. It is now clear that an important function of TADs is to restrict the interactions of

10 regulatory elements to genes within the TADs, while insulating interactions of regulatory

11 elements from neighboring domains ${ }^{3,4}$. Further evidence from our lab suggests that super-

12 enhancers, clusters of multiple enhancers that often regulate genes that determine cellular

13 identity or drive tumorigenesis ${ }^{8,9}$, are frequently insulated by and co-duplicated with strong

14 TAD boundaries in cancer ${ }^{10}$. TAD boundaries are enriched in binding of structural proteins (e.g.

15 CTCF, cohesin) ${ }^{11}$. Cohesin-mediated, convergently oriented CTCF-CTCF structural loops are

16 essential for the organization of the genome into TADs ${ }^{12-14}$. Recent studies have shown that

17 abrogation of CTCF binding or inversion of its orientation in boundary regions can change TAD

18 structure, reconfigure enhancer-promoter interactions by re-establishing loops ${ }^{15}$ and lead to

19 aberrant gene activation and developmental defects ${ }^{1,16}$.

21 In light of these reports, our understanding of how changes in chromatin organization contribute

22 to cancer pathogenesis remains largely unexplored barring a few examples ${ }^{2,17,18}$. In this study,

23 using $\mathrm{T}$ cell acute lymphoblastic leukemia (T-ALL) as a model disease ${ }^{19,20}$, we investigated

24 potential reorganization of the global chromatin architecture between primary $\mathrm{T}$ cell leukemia

25 samples, leukemia cell lines and healthy $\mathrm{T}$ cell controls. Our analysis identified recurrent

26 structural TAD boundary changes and significant alterations in intra-TAD chromatin interactions

27 (TAD activity) that mirrored changes in gene expression. Both these types of alterations

28 frequently affected effectors of oncogenic NOTCH1 signaling. As a principal example of a TAD

29 boundary change, we identified a recurrent TAD boundary loss in T-ALL within the locus of a

30 key driver of T cell leukemogenesis, MYC, which facilitates long-range interactions of the MYC

31 promoter with a previously characterized NOTCH-bound super-enhancer element. Furthermore, 
1 in highlighting a direct role for NOTCH1 in organizing local chromatin architecture, inhibition

2 of NOTCH1 signaling using gamma secretase inhibitors $(\gamma \mathrm{SI}$; a specific inhibitor of

3 transmembrane proteolytic cleavage required for NOTCH1 receptor activation) significantly

4 reduced chromatin looping in a number of enhancer-promoter pairs that are sensitive to $\gamma \mathrm{SI}$

5 treatment (called "dynamic NOTCH1" sites ${ }^{21}$ ). The loss of chromatin interactions between these

6 enhancer-promoter loops was also associated with a significant reduction of the H3K27ac mark

7 at the respective enhancer locus. However, a subset of enhancer-promoter loops including the

8 MYC super-enhancer loop retained their interactions with target promoters following $\gamma \mathrm{SI}$

9 treatment, despite being bound by dynamic NOTCH1. In exploring putative co-factors that may

10 also be responsible for maintaining long range interactions, we identified CDK7 binding to be

11 enriched in $\gamma$ SI-insensitive chromatin contacts. Pharmacological inhibition of CDK7 using the

12 covalent inhibitor THZ1 significantly reduced MYC super-enhancer promoter contacts,

13 underlining the complexity of factors regulating 3D architecture. Taken together, our findings

14 provide a deeper insight into how the 3D chromatin architecture can affect the regulatory

15 landscape of oncogenes in human leukemia and suggest that some of those changes can be

16 reversed by targeted drug treatments.

18 RESULTS

19 Widespread changes in 3D chromatin landscape in human T-ALL

20 T-ALL accounts for approximately $25 \%$ of acute lymphoblastic leukemia cases ${ }^{22}$ and is

21 characterized by activating mutations in the transmembrane protein NOTCH1 in approximately

$2250 \%$ of patients ${ }^{23,24}$. NOTCH1 mutations frequently co-occur with loss of function mutations in

23 cell cycle regulators and epigenetic factors such as $C D K N 2 A$ and $E Z H 2$, respectively ${ }^{20}$. Based

24 on gene expression signatures and flow cytometry-based immunophenotyping, T-ALL is

25 classified into two main subtypes including the "canonical" T-ALL characterized by frequent

26 NOTCH1 mutations with a T cell phenotype and the early T-lineage progenitor (ETP) leukemia

27 subtype, frequently expressing stem cell and myeloid surface markers. Though the genetic

28 drivers of T-ALL are well-characterized, it has not been investigated whether $\mathrm{T}$ cell

29 transformation is associated with widespread changes in chromatin architecture. Herein, to

30 broadly assess the global changes in chromatin architecture in T-ALL, we performed in situ Hi-C

31 in nine primary peripheral blood T-ALL samples, two T-ALL cell lines (CUTLL1 ${ }^{25}$ and Jurkat 
$1{ }^{26}$ ) and peripheral blood $\mathrm{T}$ cells from three healthy donors and integrated these datasets with

2 CTCF binding, RNA expression changes and enhancer activity (Figure 1A). The Hi-C data were

3 uniformly processed across all the samples using our HiC-bench platform ${ }^{27}$. Quality assessment

4 showed alignment rates that yielded a high percentage of usable long-range read pairs in all cases

5 (Figure S1A, Table S1). As an initial comparison of our Hi-C data across all the samples, we

6 performed a Principal Component Analysis (PCA) of genome-wide "hic-ratio" insulation scores,

7 representing the insulation capacity of every genome-wide bin, derived using the HiC-bench

8 platform. Unsupervised clustering of the hic-ratio scores using the R package "Mclust" indicated

9 three distinct clusters of samples, clearly separated by the first two components (Figure 1B).

10 Cluster 1 samples were identified as mature peripheral $\mathrm{T}$ cells and separated from the T-ALL

11 samples (cluster 2 and 3) by the first principal component. To independently discern the identity

12 of clusters 2 and 3, we interrogated the expression pattern of these samples using gene signatures

13 for canonical T-ALL and ETP-ALL derived from recent publications ${ }^{24,28,29}$. Since we had no

14 matched RNA-Seq for healthy $\mathrm{T}$ cells, we have used a publicly available set of RNA-Seq

15 datasets on healthy $\mathrm{T}$ cell donors ${ }^{30}$ (see also Table S2 for external datasets). Amongst the T-

16 ALL samples, four T-ALL samples that grouped in cluster 3 were identified to share a

17 characteristic gene signature of the ETP-ALL subtype (Figure 1C). The expression signature of

18 cluster 2 samples overlapped with that of canonical T-ALL, with a single exception displaying

19 intermediate expression of both signatures. This T-ALL sample lacked canonical NOTCH1

20 mutation but harbored activating mutation in Interleukin 7 receptor alpha chain (IL7R) ${ }^{31,32}$ and

21 deletion of PTEN ${ }^{33}$ (Table S3). T cells had no discernable expression pattern in either signature

22 as expected. Thus, the assignment of canonical T-ALL and ETP-ALL using gene expression

23 information explains the Hi-C insulation score variation between clusters 2 and 3 (Figure 1D).

24 To further confirm the heterogeneity of the T-ALL samples from the Hi-C data, we calculated

25 matrix-wide stratum-adjusted correlation coefficients using HiCRep ${ }^{34}$ between the Hi-C contact

26 matrices of all possible pairs of samples. T cell Hi-C samples from the three individual healthy

27 donors highly correlated with each other (Figure S1B). Similarly, all the canonical T-ALL

28 samples showed higher correlations to each other, including cross-comparisons of primary

29 samples versus cell lines, and lower correlations to the normal samples. The correlation among

30 ETP-ALL samples was on average higher when compared with T-ALL samples (Figure S1B), 
1 further supporting genome-wide variations in 3D architecture between the $\mathrm{T}$ cells and T-ALL

2 samples and also between the two T-ALL subtypes.

To better characterize the individual differences in 3D architecture that underlie this separation of the leukemic versus non-leukemic samples, we examined the compartmentalization of the genome between the three clusters of Hi-C samples. To this end, we utilized the c-score tool ${ }^{35}$ to determine compartment scores and integrated H3K27ac ChIP-Seq data for CUTLL1 (T-ALL),

8 Loucy (ETP-ALL) and T cells with resulting compartment scores to assign active (A) and inactive (B) compartments. First, we performed a PCA on genome-wide compartment scores, which showed a similar separation of T cells, T-ALL and ETP-ALL (Figure S1C) as observed

11 before with the genome-wide insulation scores. We further identified compartment shifts that are common to both T-ALL sub-types when compared with T cells (411 A to B; 134 B to A), as well

13 as smaller sets of compartment shifts unique to each T-ALL (40 A to B; 39 B to A) and ETP-

14 ALL (87 A to B; $108 \mathrm{~B}$ to A), again highlighting the inter-sample variations among T-ALL

15 subtypes (Figures 1E, S1D). Collectively, these data show that changes in 3D chromosomal

16 landscapes can occur in transformed leukemia cells and can help differentiate between related

17 subtypes of human leukemia.

\section{Intra-TAD activity changes affect downstream effectors of T-ALL pathogenesis}

20 We then focused on all common TADs between T cells and T-ALL that are found within the 21 transcriptionally active A compartment in either T cells or T-ALL. We defined the "intra-TAD 22 activity" as the average of all normalized interaction scores of all interactions within the 23 particular TAD (see Methods for details). Differences in the intra-TAD activity score across the 24 selected TADs were determined by comparing the fold-change of average intra-TAD activity (as 25 described above) between T cells and T-ALL as well as a paired t-test per interaction-bin per 26 TAD followed by multiple testing correction. This approach approximates regulatory enhancer27 promoter loops and structural loops that may discern transcriptional activity of each TAD 28 between $\mathrm{T}$ cells and T-ALL. The comparison of intra-TAD activity between canonical T-ALL 29 samples and controls identified several statistically significant gains and losses in T-ALL 30 (Figure 2A; FDR $<0.1$, TAD activity $\log 2$ fold-change > 0.58 / fold-change > 1.5). As a 31 negative control, we performed the same comparison between two independent $\mathrm{T}$ cell samples, 
1 which revealed no changes by applying the same thresholds (Figure 2B; FDR < 0.1, TAD

2 activity $\log 2$ fold-change > 0.58). Furthermore, the observed TAD activity changes were highly

3 similar across all the T-ALL samples (Figure 2C), with some expected heterogeneity between

4 tumors. In order to rule out that these changes were merely influenced by shifts from an active to

5 inactive compartment or vice versa, we have integrated compartment shifts that overlap our

6 differentially active TADs. We found that only $\sim 15-18 \%$ of the identified intra-TAD activity

7 changes can be explained by concomitant compartment shifts, with the majority falling in the A

8 compartment in both T cells and T-ALL samples, thus underlying a different mechanism than

9 compartment regulation (Figure S2A).

11 Because cancer genomes often show mutational aberrations and copy-number variants (CNVs),

12 we investigated the impact of such aberrations on our intra-TAD activity analysis. We used

$13 \mathrm{HiCnv}^{36}$, a tool to detect potential CNVs reliably solely from Hi-C data. Following this

14 approach, we only found between zero and nine CNVs per T-ALL sample (Figure S2B). To map

15 genetic alterations more precisely, we performed whole-genome sequencing in two selected T-

16 ALL samples and called CNVs and tandem-duplications genome-wide. We overlapped CNVs

17 (separated by gain/loss vs the linear genome) and tandem duplications with increased/decreased

18 intra-TAD activity, respectively. We found no $\mathrm{CNV} /$ tandem duplication within any reported

19 differentially active TAD (Figure S2C). Furthermore, no CNV/tandem duplication occurred

20 within the TAD boundaries adjacent to differentially active TADs, which might have influenced

21 CTCF binding (Figure S2D). We were also interested whether single nucleotide variants (SNVs)

22 detected from WGS are enriched in any differential TAD-activity category. Indeed, for both T-

23 ALL samples profiled we found a minor but significant increase in SNVs per Mb in gained intra-

24 TAD activity in T-ALL when compared to stably active TADs (Figure S2E), which could

25 potentially lead to enhancer/transcriptional upregulation and impact the 3D interactions within

26 TADs. Thus, we conclude that our analysis is not impacted by CNVs but to a modest degree by

27 SNVs, with the majority of 3D chromatin alterations potentially being epigenetically regulated.

29 To further characterize the identified differential intra-TAD activity, we integrated genome-wide

30 binding of the insulator protein CTCF (ChIP-Seq) from T cells and leukemic samples with our

31 Hi-C datasets. Interestingly, the changes in intra-TAD activity strongly correlated with changes 
1 in the binding of CTCF at the boundaries of the differentially active TADs. Thus, a stronger

2 insulation by CTCF is associated with stronger intra-TAD activity (Figure 2D), suggesting that

3 highly active TADs are strongly insulated from adjacent TADs. Next, to investigate whether

4 these CTCF binding-associated changes in intra-TAD interactions are also associated with

5 changes in gene expression, we performed differential expression analysis (canonical T-ALL vs.

6 normal) and integrated the results with the differentially active TADs. To this end, we extracted

7 all expressed genes (FPKM > 1) falling into differentially/stably active TADs and calculated

8 gene expression fold-changes between the leukemic sample and their normal counterparts.

9 Following the hypothesis of a positive correlation between intra-TAD activity and gene

10 expression, we observed that increased chromatin interactions in T-ALL significantly associated

11 with positive fold-changes in gene expression, whereas decreased intra-TAD activity in T-ALL

12 correlated with negative fold-changes in gene expression when compared to gene expression

13 changes within stable TADs (Figure 2E). We then overlapped these highly correlating changes

14 of intra-TAD activity, CTCF insulation and gene expression with cell-type specific super-

15 enhancer activity in T-ALL and T cells. We defined super-enhancers for naïve T cells and the T-

16 ALL cell-line CUTLL1 with the ROSE algorithm ${ }^{9}$ applied on H3K27ac ChIP-Seq data and

17 identified cell-type specific super-enhancers. We found a significant enrichment of T-ALL

18 specific super-enhancers in the TADs that gained activity in T-ALL and, vice versa, a significant

19 enrichment of $\mathrm{T}$ cell specific super-enhancers in TADs that lost activity (Figure 2F). Taken

20 together, these results demonstrate the existence of a number of changes in intra-TAD activity in

21 T-ALL cells and associated TAD activity alterations, CTCF binding, mRNA expression and

22 super-enhancer activity.

24 To investigate the impact of genetic alterations on CTCF binding, we interrogated again the

25 WGS from two primary T-ALL samples. We found that genome-wide differential CTCF binding

26 was not particularly impacted by SNVs overlapping with CTCF binding motifs when compared

27 to stable CTCF binding (Figure S2F). We have observed small variances when selectively

28 comparing differential CTCF binding overlapping with SNVs within TAD boundaries of

29 differentially active TADs. However, the majority of differential CTCF binding within altered

30 TAD boundaries was not affected by SNVs (Figure S2G). 
1 Our comparison of changes in TAD activity and super-enhancer firing suggests that 3D 2 chromosomal changes should occur in loci that are important for T-ALL pathogenesis, including 3 genes that are NOTCH1 targets and over-expressed in T-ALL samples. One such gene is the 4 adenomatous polyposis coli downregulated 1 (APCDD1), a membrane bound glycoprotein that is 5 overexpressed in T-ALL patient samples and is a NOTCH1 target gene that is significantly 6 downregulated following inhibition of NOTCH1 signaling (dynamic NOTCH1 target) by $\gamma \mathrm{SI}^{21}$.

7 Our Hi-C data showed that APCDD1 was present in a TAD that gained activity in T-ALL 8 relative to control T cells (Figure 2G, I). The increased TAD activity was common among all the T-ALL samples, concomitant with widespread activation of enhancer elements specific to TALL (Figure 2H). The gain of TAD activity also correlated with increased expression of APCDD1 in T-ALL samples relative to control T cells (Figure 2I). Another example of a TALL-specific increase in intra-TAD activity, enhancer activity and gene expression is Ikaros family gene IKZF2 (Helios). IKZF2 overexpression in hematopoietic progenitors results in T cell

14 developmental arrest ${ }^{37}$. We were able to identify a T-ALL specific super-enhancer within the same TAD, as well as a significantly increased gene expression in T-ALL compared to normal T cells (Figure S3A, B, C). In contrast, among the TADs that lost activity in T-ALL, we identified the gene CYLD, a deubiquitinating enzyme, and a known repressor of NF-kB signaling and a putative tumor suppressor in T-ALL. Previous work from our lab and others identified that constitutive activation of NOTCH1 triggers the NF-kB pathway in T-ALL and the mechanism of NOTCH1 dependent activation of NF-kB signaling is through the repression of CYLD ${ }^{38,39}$. CYLD is a negative regulator of IKK, which promotes NF-kB signaling. We found significant

22 loss of interactions in the TAD that harbors CYLD in all profiled T-ALL samples (Figure S3D,

23 E). The loss of TAD activity also correlated with the decreased expression of CYLD in T-ALL

24 samples (Figure S3F).

\section{Intra-TAD activity distinguishes between T-ALL subtypes}

27 Finally, to investigate subtype specific differences in TAD activity, we evaluated the intra-TAD 28 differences between the canonical T-ALL and ETP-ALL samples. We performed both individual 29 comparisons of T-ALL and ETP-ALL versus untransformed T cells, and also directly compared 30 the intra-TAD activity between T-ALL and ETP-ALL. These comparisons identified both 31 common changes in T-ALL and ETP-ALL when compared to T cells, but also disease-specific 
1 alterations that reflect both the common lineage but also different stages of maturation arrest of

2 the two subtypes (Figure $\mathbf{S 4 A , B}$ ). Integration of gene expression changes with differentially

3 active TADs again indicated significant correlations of intra-TAD activity changes with

4 expression changes between ETP-ALL and T cells (Figure S4C). Similarly, we found significant

5 correlation of expression changes with intra-TAD activity changes between ETP-ALL and

6 canonical T-ALL (Figure S4D), highlighting the impact of 3D architecture on gene expression

7 changes between T-ALL subtypes.

Identification of recurrent TAD insulation changes in T-ALL

10 Following the identification of differences in intra-TAD activity, we further investigated TAD

11 boundary changes between normal $\mathrm{T}$ cells and T-ALL samples. We performed a global TAD

12 insulation alteration analysis on all pairs of adjacent TADs found in $\mathrm{T}$ cells (revealing TAD

13 boundary losses) and, vice versa, on all pairs of adjacent TADs found in T-ALLs (revealing

14 TAD boundary gains) (Figure 3A). A TAD boundary loss was defined as an increase in inter-

15 TAD interactions accompanied by intra-TAD changes as well as loss of CTCF binding between

16 two adjacent T cell TADs leading to a TAD "fusion" event. Conversely, a TAD boundary gain

17 was defined as the formation of two distinct TADs (TAD "separation") with decreased inter-

18 TAD interactions, changes in intra-TAD interactions as well as increased CTCF binding in T-

19 ALLs (Figure 3A) (see Methods for details). TAD fusion or separation events can change local

20 regulatory landscapes by altering insulation of genes from nearby regulatory elements. In order

21 to estimate a false discovery rate for our findings, we have performed the analysis first between

22 all pairwise $\mathrm{T}$ cell comparisons as well as between T cells and T-ALL without integrating CTCF

23 information (Figure 3B). Importantly, the pairwise T cell comparisons (obtained from different

24 donors) identified only a few TAD boundary alterations between each other that could be due to

25 the potential heterogeneity between the individual donors. However, if we consider all such

26 insulation changes between the $\mathrm{T}$ cell samples as false-positives, we estimated an approximate

$2710.77 \%$ false discovery rate (FDR) for TAD boundary changes in T-ALL compared to T cells.

28 Next, we integrated CTCF binding information for the T-ALL versus $\mathrm{T}$ cell comparisons

29 (Figures 3C, D). To further investigate the heterogeneity of T-ALL samples for such TAD

30 reorganization events and as an independent validation, we calculated the hic-ratio insulation

31 score for all TAD boundary alterations found between T-ALLs and T cells. The hic-ratio score 
1 only acts as a local approximation of insulation and may miss long-range interaction changes of

2 large TADs as interrogated specifically in our TAD boundary alteration analysis. The hic-ratio

3 insulation score was on average significantly increased/decreased for TAD boundary

4 gains/losses, respectively, across all T-ALL samples (Figure 3E, F).

Lastly, using WGS data, we found only two TAD boundaries displaying increased/decreased insulation capacity in T-ALL overlapping with either genomic deletions or insertions, however,

8 none of the indels was directly overlapping with CTCF binding motifs of differential CTCF

9 binding sites (Figure S5A). We furthermore identified two genomic inversions (potentially

10 leading to aberrant CTCF orientation as previously reported ${ }^{15,40}$ ) that overlapped reported TAD

11 boundary insulation alterations (Figure S5B). However, none of them affects genomic loci that 12 contain relevant T-ALL genes.

\section{A recurrent TAD fusion event permits $M Y C$ promoter/super-enhancer looping}

15 MYC is widely up-regulated in T-ALL and is one of the main oncogenes activated downstream 16 of NOTCH1 signaling, contributing to metabolic rewiring, cell growth and proliferation ${ }^{41,42}$.

17 Intriguingly, we identified a recurrent TAD fusion event in the MYC locus in all T-ALL samples 18 studied (both primary and cell lines) compared to untransformed T cells (Figure 4A). The TAD 19 fusion event in all T-ALL samples led to a strong increase in inter-TAD interactions compared to 20 control $\mathrm{T}$ cells. We then investigated whether this observed increase in inter-TAD interactions 21 and the fusion of the two TADs into one larger TAD is a result of loss of CTCF-mediated 22 insulation at the T cell specific TAD boundary. CTCF ChIP-Seq and ChIP-qPCR data confirmed 23 CTCF binding at the TAD boundary in normal $\mathrm{T}$ cells, and an almost complete abrogation of 24 CTCF binding at this boundary across the T-ALL samples (Figure 4B, S6A). This loss of CTCF 25 binding was not due to mutation of the CTCF binding site in T-ALL (Figure S6B) or DNA 26 methylation changes within the CTCF binding site countering CTCF binding in T-ALL (data not 27 shown). Furthermore, 5-azacytidine treatment leading to global DNA de-methylation showed no 28 restoration of CTCF binding in CUTLL1 cells (Figure S6C). Instead, ATAC-Seq data indicated 29 a significant decrease in chromatin accessibility of the CTCF binding site in T-ALL (Figures 30 4B, S6D). 
1 In T-ALL, MYC transcription is controlled by distant 3D interactions with a long stretch of 2 enhancers, including the previously characterized N-Me/NDME element ${ }^{43,44}$. This particular 3 enhancer element is strongly bound by NOTCH1 (Figure 4B). As a result of the here reported 4 TAD fusion event, the $M Y C$ promoter and its super-enhancer, that are separated by strong 5 insulation in $\mathrm{T}$ cells, are now in close spatial proximity within the same TAD in leukemic samples (Figure 4A, B). To further validate whether this TAD boundary change and resulting TAD fusion increases interactions of the $M Y C$ promoter with the distal super-enhancer at high resolution, we performed 4C-Seq analysis using the $M Y C$ promoter as viewpoint in the leukemia samples and normal T cells. 4C-Seq confirmed the interactions between the MYC promoter and

10 the super-enhancer element in primary T-ALL samples and CUTLL1, whereas in untransformed

11 T cells, no such interaction was observed (Figure 4C, S7A). This was further highlighted by the 12 strong insulation at the lost TAD boundary provided by the binding of CTCF in $\mathrm{T}$ cells. 13 Interestingly, our analysis showed that the strongest and most significant interactions specifically 14 overlap with H3K27ac ChIP-seq peaks throughout the entire super-enhancer element, including 15 an uncharacterized putative center enhancer element (from here on termed MYC-CEE) and the 16 BDME/BENC enhancer cluster recently identified to be essential for normal hematopoiesis and 17 AML pathogenesis (Figure 4C, S7A) ${ }^{45,46}$. In agreement with our 3D chromosomal interaction 18 data, MYC was overexpressed in our T-ALL cohort compared to T cells from healthy donors 19 (Figure 4D). Furthermore, we independently validated the differential 3D localization of $M Y C$ 20 and the super-enhancer between T cells and T-ALL using 3D Fluorescence in situ hybridization 21 (FISH) with probes targeting the MYC promoter and the center super-enhancer element MYC22 CEE. Inter-probe distance was significantly higher in T cells compared to T-ALL cells (the 23 CUTLL1 cell line) consistent with the increased interactions in CUTLL1 identified by 4C-Seq 24 (Figure 4E). To confirm the CTCF-mediated insulation of the MYC TAD in $\mathrm{T}$ cells, we 25 disrupted the CTCF binding site in normal T cells using CRISPR (clustered regularly interspaced 26 short palindromic repeats) mutation (Figure S8A). We achieved $\sim 92 \%$ of cells harboring indels 27 of varying sizes located within the CTCF motif (Figure S8B). Mutations of the CTCF motif in T 28 cells resulted in significantly decreased CTCF binding in the edited T cells (Figure S8C) and 29 marginally increased MYC expression (Figure S8D). The decreased binding of CTCF protein 30 was accompanied by significantly reduced interactions between the MYC promoter and the 31 CTCF bound TAD boundary region in edited T cells compared to WT T cells (Figure S8E). 
1 Pharmacologic NOTCH1 inhibition leads to a decrease of $3 \mathrm{D}$ interactions in a group of 2 NOTCH1-regulated loci

3 Our analysis revealed widespread changes in global TAD structure and intra-TAD activity

4 affecting important genes in T-ALL. However, the question whether oncogenic drivers, such as

5 NOTCH1, play a direct role in these changes and whether their inhibition can reverse these

6 changes remains open. To address this, we performed in situ Hi-C analysis in CUTLL1 cells

7 treated with the NOTCH pathway inhibitor targeting the gamma secretase complex $(\gamma \mathrm{SI})$ for $72 \mathrm{~h}$

8 as performed previously ${ }^{21,41}$. $\gamma$ SI selectively and efficiently inhibits NOTCH1 signaling and has

9 strong anti-leukemic effects ${ }^{42,47}$. Hi-C analysis following $\gamma$ SI treatment did not reveal any

10 significant changes either in intra-TAD activity (Figure S9A) or reversal of TAD boundary

11 insulations (Figure S9B). This was not surprising since NOTCH1, a bona fide signal-dependent

12 transcription factor, was not expected to impact global chromatin architecture. However, it was

13 previously shown that about $90 \%$ of NOTCH1 binding sites that are sensitive to $\gamma$ SI treatment

14 (i.e. dynamic NOTCH1 sites) are localized outside promoters in putative distal enhancer

15 elements. These dynamic NOTCH1-occupied enhancers also showed significant changes in

$16 \mathrm{H} 3 \mathrm{~K} 27 \mathrm{ac}$ signal following NOTCH1 inhibition ${ }^{21}$. We investigated whether chromatin

17 interactions between dynamic NOTCH1-occupied enhancers and neighboring promoters were

18 altered following $\gamma$ SI treatment. To this end, we first profiled H3K27ac following $\gamma$ SI treatment

19 and categorized all non-promoter H3K27ac peaks as either stable peaks that do not change

20 following $\gamma \mathrm{SI}$ treatment or those that have either a strong reduction or increase in $\mathrm{H} 3 \mathrm{~K} 27 \mathrm{ac}$

21 signal following $\gamma \mathrm{SI}$ treatment (Figure 5A). As previously observed, the H3K27ac peaks that

22 had a strong reduction in signal following $\gamma \mathrm{SI}$ treatment were also significantly enriched for

23 dynamic NOTCH1 binding when compared to stable or increased H3K27ac signals ${ }^{21}$ (Figure

24 5B). An increase in $\mathrm{H} 3 \mathrm{~K} 27 \mathrm{ac}$ upon $\gamma \mathrm{SI}$ treatment is thus likely a downstream effect of NOTCH1

25 inhibition. To connect NOTCH1 pathway inhibition, changes in H3K27ac and 3D looping, we

26 used Hi-C data following $\gamma$ SI treatment to quantify changes in chromatin interactions of

27 H3K27ac-enriched chromatin loops identified by H3K27ac HiChIP in CUTLL1 ${ }^{48}$. Our HiChIP

28 data showed strong enrichment of promoter-enhancer interactions as represented by a virtual 4C

29 analysis using the MYC promoter as virtual viewpoint, as well as reliable detection of such loops

30 from our pipeline (Figure S9C). Dynamic NOTCH1-bound enhancers with reduced H3K27ac 
1 levels following $\gamma \mathrm{SI}$ treatment showed the strongest loss of chromatin interactions with connected genes (Figure 5C). Interestingly, dynamic NOTCH1-bound enhancers with no change in $\mathrm{H} 3 \mathrm{~K} 27 \mathrm{ac}$ signal on average remained in stable contact with nearby promoters. To correlate

4 these changes in chromatin interaction with the dynamics of NOTCH1-dependent transcription, 5 we also performed global run of sequencing (GRO-Seq) ${ }^{49}$ to measure nascent transcription 6 following NOTCH1 inhibition by $\gamma$ SI and release after inhibitor "wash off" for increasing time 7 intervals. Interestingly, the regulatory enhancer-promoter contacts most sensitive to $\gamma \mathrm{SI}$ 8 treatment were among genes that showed significant response in transcription to NOTCH1 9 inhibition and release after $\gamma$ SI wash off (Figure 5D).

11 To further validate the changes in interactions among the NOTCH1-sensitive enhancer-promoter 12 loops, we performed 4C-Seq analysis for two previously characterized NOTCH1 T-ALL targets, 13 LUNAR1 and APCDD1. LUNAR1 is a long non-coding RNA that we have previously identified 14 as a cis regulator of the expression of the neighboring $I G F 1 R$ gene. This regulation is achieved 15 by chromatin looping of LUNARI promoter with an intronic enhancer in $I G F 1 R^{50,51}$. 4C-Seq using LUNARI promoter as viewpoint identified strong interactions between LUNARI promoter

17 and the IGF1R enhancer element. However, the interaction decreased significantly following 18 NOTCH1 inhibition (Figure 5E, S9D). The decrease in interaction also correlated with reduction in H3K27ac signal at the enhancer element and decreased expression of LUNAR1 (Figure 5E, S9D). Similarly, in the APCDD1 locus, 4C-Seq using an APCDD1 enhancer with dynamic NOTCH1 and reduced H3K27ac signal as viewpoint, we identified decreased interaction between the enhancer and the promoter of APCDD1. These changes also correlated with reduced H3K27ac in the enhancer and reduced expression of APCDD1 (Figure 5F, S9E). These results

24 suggest that pharmacologic NOTCH1 inhibition can affect activity (as defined by H3K27ac 25 levels) of dynamic NOTCH1-bound enhancers and that 3D interactions in such enhancers are significantly diminished.

28 However, our analysis revealed that a subset of dynamic NOTCH1-regulated loci was not 29 associated with either H3K27ac loss or reduced long-range chromatin interactions following $\gamma$ SI 30 treatment. Based on this observation, we classified enhancers with reduced NOTCH1 binding 31 and H3K27ac levels upon $\gamma$ SI treatment as $\gamma$ SI-sensitive enhancers, and enhancers with only 
1 reduced NOTCH1 binding as $\gamma \mathrm{SI}$-insensitive enhancers (Figure S9F). Interestingly, $\gamma \mathrm{SI}$ 2 sensitive enhancers tend to be shorter in length than $\gamma$ SI-insensitive enhancers (Figure S9G). For 3 example, our initial 4C analysis had identified contacts of the $M Y C$ promoter with three enhancer 4 clusters. 4C-Seq analysis detected no significant decrease in the frequency of interactions 5 between the MYC promoter and all of the three enhancer clusters following $\gamma$ SI treatment 6 (Figure S10A, B), although $\gamma$ SI treatment reduced MYC expression (to approximately half by $7 \quad$ qPCR and western blot; Figure S10B, C) and NOTCH1 binding at the MYC super-enhancer 8 (Figure S10A, B). We also noticed only moderate changes in the H3K27ac distribution within 9 the NOTCH1-bound enhancer elements after $\gamma$ SI treatment (Figure S10A, B). Also, the critical 10 CTCF binding within the TAD boundary of MYC was not restored upon $\gamma$ SI treatment (Figure 11 S10D). Thus, despite the downregulated MYC mRNA expression and the loss of NOTCH1 12 binding, pharmacological inhibition of NOTCH1 signaling was not able to alter 3D interactions 13 in this locus. As an additional example, a dynamic NOTCH1-bound enhancer looping to the

$14 I K Z F 2$ promoter did not lose interactions following $\gamma$ SI treatment (Figure S10E, F), suggesting 15 that NOTCH1 binding is critical for maintaining enhancer-promoter contacts in only a subset of 16 such loops and additional chromatin regulators may play a role in maintaining chromatin 17 interactions of the $\gamma$ SI-insensitive loops.

\section{CDK7 inhibition targets $\gamma$ SI-insensitive enhancer-promoter loops}

20 To further understand the differential sensitivity of the dynamic NOTCH1-bound enhancers, we

21 performed a differential binding analysis using LOLA ${ }^{52}$ between the $\gamma$ SI-sensitive and 22 insensitive enhancers using publicly available ChIP-Seq datasets from the LOLA database. 23 Among the chromatin regulators and transcription factors with available ChIP-Seq datasets in T-

24 ALL cells, we found cyclin-dependent kinase 7 (CDK7) binding to be significantly enriched in 25 the $\gamma$ SI-insensitive enhancers compared to the sensitive enhancers (Figure 6A). CDK7 controls 26 the dynamics of transcriptional initiation by the phosphorylation of RNA polymerase II CTD at 27 Ser-5 and Ser-7 ${ }^{53}$. CDK7 inhibition has been particularly linked to suppression of super28 enhancer linked oncogenic transcription ${ }^{54-56}$. To globally assess the role of CDK7 binding in the 29 maintenance of $\gamma$ SI-insensitive enhancer-promoter loops, we performed in situ Hi-C analysis in 30 CUTLL1 cells treated with the CDK7 inhibitor THZ1, which was previously demonstrated to 31 have strong anti-leukemic activity ${ }^{54}$. As before, we first profiled H3K27ac levels following 
1 THZ1 treatment by ChIP-Seq and categorized all non-promoter H3K27ac peaks as either 2 insensitive with stable $\mathrm{H} 3 \mathrm{~K} 27 \mathrm{ac}$ signal, peaks that have a strong reduction (THZ1 lost enhancer) 3 or peaks with an increase (THZ1 gained enhancer) in H3K27ac signal following CDK7

4 treatment (Figure 6B). Globally, as previously observed in the $\gamma \mathrm{SI}$ treatment, enhancers with a 5 strong reduction in signal of $\mathrm{H} 3 \mathrm{~K} 27 \mathrm{ac}$ peaks had a correlative strong reduction in long-range

6 chromatin interactions to target promoters, whereas the insensitive enhancers with no change in

7 H3K27ac signal following THZ1 treatment, neither gained nor lost chromatin interactions on a 8 global scale (Figure 6C).

10 To further test the role of CDK7 in maintaining DNA loops, we performed high-resolution 4C11 Seq following THZ1 treatment in the previously identified $\gamma$ SI-insensitive MYC and IKZF2 loci.

12 We observed a significant decrease in the interaction between both N-Me/NDME and MYC$13 \mathrm{CEE}$ and the $M Y C$ promoter following the CDK7 treatment (Figure 6D, S11A). These changes 14 were accompanied by a significant decrease of the H3K27ac signal and MYC expression 15 (Figure 6D, S11A, S11B). Finally, no significant gain in the binding of CTCF to the TAD 16 boundary was observed, suggesting that the described loss of the promoter-enhancer interaction occurs independently of CTCF binding (Figure S11C). Additionally, DNA FISH analysis confirmed a significant increase in 3D distance between the $M Y C$ enhancer and promoter probes

19 following THZ1 treatment (Figure S11D). We also performed THZ1 treatment followed by 4C-

20 Seq on the MYC promoter viewpoint in Jurkat cells, which display the same TAD fusion and 21 super-enhancer activity as CUTLL1. Our results indicate a global reduction of interactions 22 throughout the entire super-enhancer element in Jurkat (Figure S11E, F). Furthermore, similar 23 loss of both enhancer activity and enhancer-promoter interaction was also observed in the IKZF2 24 locus as shown by the H3K27ac ChIP-Seq data and 4C-Seq in CUTLL1 cells (Figure 6E, 25 S11G). Overall, these studies demonstrate that targeting a single transcription factor (NOTCH1) 26 is able to affect only a subset of 3D promoter-enhancer interactions associated with dynamic

27 NOTCH1. Additional factors such as CDK7 can maintain contacts in a subset of NOTCH128 insensitive enhancers in T-ALL. Furthermore, changes in H3K27ac levels emerge as a reliable 29 indicator of gain or loss of chromatin interactions following these drug treatments. 


\section{DISCUSSION}

2 Despite the intense focus on the regulatory role of TADs in human disease, it remains largely 3 unexplored whether TAD boundary or intra-TAD activity changes are important for tumor

4 initiation and/or maintenance. Indeed, aberrant activation of cancer drivers by enhancer hijacking 5 remains the primary known mechanism linking 3D structural changes to oncogenic 6 transformation 2,3,57,58. Our studies further these findings using T-ALL as a model. They 7 highlight the underlying complexity of factors regulating the 3D landscape in human leukemia 8 with notable variations among different leukemia sub-types and suggest that drugs with reported 9 anti-leukemic activity partially reverse 3D interactions in selected loci, potentially accounting for 10 the anti-leukemogenic effects of these drugs.

12 Frequent loss of TAD boundary insulation has been previously observed in human cancer, 13 including T-ALL ${ }^{58}$. Consistent with these findings, we identify here a TAD boundary loss 14 within the MYC locus that resulted in loss of insulation and enhancer hijacking. MYC is an 15 important downstream target of NOTCH1 that activates anabolic pathways to sustain 16 proliferation induced by constitutive NOTCH1 activation ${ }^{41,42}$. Our observations suggest that 17 MYC upregulation in leukemic cells is associated with differences in local chromatin 18 architecture. At this point, it is not clear what causes the loss of CTCF binding within the TAD 19 boundary in T-ALL cells, although our preliminary studies have excluded a role for DNA 20 methylation and somatic mutations within the CTCF motif. Interestingly, using ATAC-Seq data, 21 we found that the CTCF site is accessible in T cells but displayed greatly reduced accessibility in 22 T-ALL cells suggesting differential chromatin accessibility as a potential mechanism of 23 regulating CTCF binding. In support of this hypothesis, a recent report identified chromatin 24 accessibility around CTCF binding sites to be greatly reduced during the interphase to pro25 metaphase transition ${ }^{59}$. Furthermore, reduced chromatin accessibility correlated with reduced 26 CTCF binding and loss of TAD and CTCF loop structures in pro-metaphase. In addition to the 27 lost CTCF boundary in T-ALL, we also observed an increase in CTCF binding with the same 28 orientation (facing into the TAD and towards MYC) downstream of the super-enhancer. Such 29 clusters of CTCF surrounding super-enhancers have recently been described as super-anchors 30 forming so-called stripes to ensure super-enhancer mediated regulation of nearby genes ${ }^{60}$. 
1 Besides the boundary gains and losses that affect TAD structures, we also found prevalent 2 changes in intra-TAD activity among the TAD structures that remained constant between T-ALL 3 and $\mathrm{T}$ cells. The changes in intra-TAD activity correlated with expression changes, 4 enhancer/super-enhancer activity and insulation mediated by CTCF binding in those TAD 5 boundaries, which appeared to be independent of compartment shifts. Supporting a prominent role for intra-TAD activity changes in modulating gene expression, recent studies tracking 3D chromatin modifications during the course of developmental processes such as embryonic stem

8 cell differentiation and neural development did not identify major structural differences in TAD 9 boundaries, but instead identified significant changes in interactions within TADs and sub-TADs 10 that correlated with transcriptional levels and epigenetic states ${ }^{61,62}$. Furthermore, in line with our 11 findings, negative correlations of intra-TAD interactions with repressive histone marks have 12 been reported in EZH2 mutant lymphomas ${ }^{63}$. Herein, our observation suggests that expression 13 changes observed following tumor initiation are frequently associated with correlative changes in 14 intra-TAD activity, CTCF insulation and enhancer activation.

16 Finally, we also addressed the direct role of oncogenic NOTCH1 in organizing the 3D 17 chromosomal landscape associated with leukemic transformation and to what extend the 18 identified changes can be reversed by inhibiting NOTCH-signaling. NOTCH signaling inhibition 19 is a powerful means to inhibit leukemic growth of NOTCH1 activated T cells ${ }^{47,64}$. The effects of

$20 \gamma$ SI were reported to be selective to the dynamic NOTCH1 sites, which are predominantly 21 located within enhancers ${ }^{21,44}$. Such dynamic NOTCH1 sites are also associated with a decrease 22 in enhancer activity following $\gamma$ SI treatment. These findings prompted us to further investigate 23 the impact of NOTCH1 inhibition on the remodeling of the 3D landscape in leukemia. Our study 24 showed that NOTCH1 inhibition using $\gamma$ SI had no effect on global 3D chromatin structure and 25 intra-TAD activity, but partially targeted enhancer-promoter interactions in selected NOTCH126 regulated loci. More specifically, we identified enhancer-promoter loops of dynamic NOTCH127 bound enhancers that were also associated with a decrease in H3K27ac following $\gamma$ SI treatment 28 were particularly sensitive to NOTCH1 inhibition. These results concur with a recent report that 29 demonstrated a role for NOTCH1 in facilitating specific long-range interactions in triple30 negative breast cancer and mantle cell lymphoma models ${ }^{18}$. 
1 In further understanding the differential importance for NOTCH1 binding in maintaining certain

2 enhancer-promoter loops but not others, we found that enhancers most sensitive to NOTCH1

3 inhibition tend to be shorter in length. The longer stretch of the insensitive enhancers might

4 enable other factors to bind and/or keep the chromatin in an open and accessible state for long-

5 range chromatin interactions ${ }^{65}$, thus offering a potential explanation for the variances in

6 promoter-enhancer looping changes we observed for the NOTCH1-targets, including MYC,

7 IKZF2, APCDD1 and LUNAR1. In agreement with this hypothesis, we found enrichment for

$8 \quad$ CDK7 binding in $\gamma$ SI-insensitive enhancers over $\gamma$ SI-sensitive enhancers. CDK7 inhibition has

9 shown significant effects in hematological malignancies and other cancer ${ }^{54-56}$. Pharmacological

10 inhibition of CDK7 by THZ1 resulted in widespread decrease in enhancer activity as quantified

11 by H3K27ac levels. Enhancers with strong reduction of H3K27ac were also associated with

12 correlative decrease in enhancer-promoter contacts. In turn, enhancer activity together with

13 chromatin looping in the $\gamma$ SI-insensitive example loci MYC and IKZF2 have been concordantly

14 reduced upon THZ1 treatment. This clearly highlights the complexity of super-enhancer activity

15 and factors that dictate their interactions with gene promoters. Overall, our study underscores the

16 need for further investigation of factors that rewire long-range interactions especially during

17 tumorigenesis, as they could be potential targets for small molecule drug development.

19 ACKNOWLEDGEMENTS

20 We thank all members of the Aifantis/Tsirigos laboratories for discussions throughout this

21 project. A. Heguy and the NYU Genome Technology Center (supported in part by National

22 Institutes of Health (NIH)/National Cancer Institute (NCI) grant P30CA016087-30) for expertise

23 with sequencing experiments; the NYU Flow Cytometry facility for expert cell sorting; the

24 Applied Bioinformatics Laboratory for computational assistance; Genewiz for expertise with

25 sequencing experiments. This work has used computing resources at the High-Performance

26 Computing Facility at the NYU Medical Center. We would also like to acknowledge Bing Ren

27 and Anthony Schmitt for the support on the Hi-C experiments. I.A. is supported by the NIH

28 (1P01CA229086-01A1， RO1CA216421， RO1CA202025， R01CA133379， R01CA149655,

29 5R01CA173636, 1R01CA194923), the Alex's Lemonade Stand Cancer Research Foundation,

30 the Chemotherapy Foundation, The Leukemia and Lymphoma Society and the NYSTEM

31 program of the New York State Health Department. A.T. is supported by the American Cancer 
1 Society (RSG-15-189-01-RMC), the Leukemia and Lymphoma Society and the St. Baldrick's

2 Foundation. P.T. was previously supported by an AACR Incyte Corporation Leukemia Research

3 Fellowship and is currently supported by a Young Investigator Grant from Alex's Lemonade

4 Stand Cancer Research Foundation. P.N. was supported by the NCI (R00CA188293) and the

5 American Society of Hematology. P.V.V was supported by an ERC Starting Grant (639784).

6 A.T. is a Scientific Advisor to Intelligencia.AI. All other authors declare that they have no

7 competing financial interests.

\section{AUTHOR CONTRIBUTIONS}

10 A.T. and I.A. conceived, designed and supervised the study with input from A.K., P.T. and P.N.

11 A.K. designed and performed most of the computational analyses with help from A.T., S.N. and

12 C.L. P.T. and P.N. designed and performed most of the experiments with help from Y.G. X.C.,

13 H.H., S.B., J.W., T.T., Y.F., F.B., Y.Z., E.P., P.V.V., G.G.I. and T.L.. P.T., P.N. and Y.G.

14 performed Hi-C, HiChIP and 4C experiments with help from X.C., S.B., J.W. and Y.Z.. P.T.

15 performed DNA FISH with help from Y.F. and T.L. P.T. performed ChIP-Seq with help from

16 P.N., S.B. and J.W.. P.T. and H.H. performed RNA-Seq. T.T. performed GRO-Seq. A.T., I.A.

17 A.K. and P.T. wrote the manuscript with input from all authors.

\section{DATA AVAILABILITY}

20 All sequencing data created within this study was uploaded to NCBI GEO 21 (https://www.ncbi.nlm.nih.gov/geo/) and is available under the accession GSE115896.

\section{CODE AVAILABILITY}

24 All code for $\mathrm{Hi}-\mathrm{C}$ analysis is available within the previously published Hi-C bench platform 25 (https://github.com/NYU-BFX). 


\section{FIGURE LEGENDS}

2 Figure 1: In Situ Hi-C analysis identifies genome-wide 3D chromatin differences between 3 normal $T$ cells and T-ALL subtypes.

4 A) Schematic showing the overall study design.

5 B) Principal Component Analysis (PCA) of the genome-wide "hic-ratio" insulation scores

6 (defined and implemented in HiC-bench) for each Hi-C dataset identified three distinct clusters.

7 Clustering was performed using R package Mclust, with EII and VII models showing an optimal

8 separation using three clusters.

9 C) Heatmap representation of RNA-Seq results for three clusters separated by T-ALL and ETP-

10 ALL signature (rows). Gene signature was derived from RNA-Seq results from ${ }^{24,28,29}$. Heatmap

11 shows row Z-score of FPKM normalized read-counts using edgeR function rpkm.

12 D) Principal Component Analysis (PCA) of the genome-wide "hic-ratio" insulation scores as in

13 B), colored by cell type assignment with the help of RNA-Seq.

14 E) Compartment analysis using the c-score tool. Bins were assigned to A compartment with an

15 average c-score $>0.1$, B compartment was assigned with an average c-score $<-0.1$, using 16 representative $\mathrm{H} 3 \mathrm{~K} 27 \mathrm{ac}$ ChIP-Seq data for directionality (higher enrichment with $\mathrm{H} 3 \mathrm{~K} 27 \mathrm{ac}$ 17 ChIP-Seq peaks determines active compartment). Different categories of disease-specific / 18 common compartment switches were identified using unpaired two-sided t-test on c-scores 19 between T-ALL, ETP-ALL and T cells, and significant differences were identified using p-value $20<0.1$.

22 Figure 2: Intra-TAD activity changes affect downstream effectors of T-ALL pathogenesis

23 A) Volcano plot showing differential intra-TAD activity for pair-wise comparisons of $\mathrm{T}$ cells 24 versus T-ALL. Differentially active TADs were selected using log2 fold-change of average intra25 TAD activity $>0.58$ or $<-0.58$ with FDR $<0.1$ (horizontal red dotted line), and are highlighted 26 red / blue, respectively. Statistical evaluation of each TAD was performed using paired two27 sided t-test for each interaction-bin per TAD between averages of T cells and T-ALL.

28 B) Volcano plot of the same analysis as in A) between two healthy T cell Hi-C samples.

29 C) Heatmap showing average per-sample intra-TAD activity in all T-ALL samples and T cells 30 normalized by the average TAD activity across all three $\mathrm{T}$ cell samples. Rows are showing 31 differentially active / stable TADs as highlighted in A). 
1 D) Integration of CTCF binding information with TAD boundary categories from A). All CTCF

2 bindings from surrounding TAD boundaries are aggregated, and the log2 fold-change of such

3 CTCF signals between T-ALL and T cell is shown. Significant differences are calculated by an

4 unpaired one-sided t-test comparing aggregated CTCF levels from TADs with decreased /

5 increased intra-TAD activity with aggregated CTCF levels from stable TADs, assuming a

6 positive correlation between CTCF binding and intra-TAD activity.

7 E) Integration of RNA-Seq (minimum per-gene expression filter FPKM > 1) within TADs with

8 decreased / increased intra-TAD activity. For each such gene, the respective $\log 2$ fold-change in

9 expression between $\mathrm{T}$ cells and T-ALL taken from RNA-Seq is shown. Significant global

10 differences are calculated by an unpaired one-sided t-test comparing genes from TADs with

11 decreased / increased intra-TAD activity with genes from stable TADs, assuming a positive

12 correlation between expression and intra-TAD activity changes.

13 F) Super-enhancer integration with differentially active TADs. Only super-enhancers found

14 mutually exclusive in either T cells or T-ALL were used. Enrichment score was calculated as

15 observed overlap between super-enhancers and differentially active / stable TADs over expected

16 background (cell-type specific super-enhancers in all genome-wide TADs). Statistical

17 enrichment compared to background (cell-type specific super-enhancers in all genome-wide

18 TADs) was calculated using two-sided Fisher's exact test.

19 G) Hi-C interaction heatmaps (first row) showing the APCDDl containing TAD (black

20 rectangles). Second row shows heatmaps of per-bin $\log 2$ fold-change interactions when

21 compared to $\mathrm{T}$ cell 1.

22 H) H3K27ac and NOTCH1 ChIP-Seq tracks for the APCDD1 locus, shown as fold-enrichment 23 over input.

24 I) Quantifications for intra-TAD activity (left; as highlighted in G)) and expression of APCDD1

25 (right). Statistical evaluation for intra-TAD activity was performed using paired two-sided t-test

26 of average per interaction-bin for APCDD1 TAD between T cells and T-ALL, followed by

27 multiple testing correction (see methods). APCDD1 expression was determined by RNA-Seq and

28 shown as $\log 2$ FPKM for T cells and T-ALL samples; normalization and statistical evaluation

29 was performed using edgeR followed by multiple testing correction. 
1 Figure 3: TAD boundary insulation analysis reveals changes in insulation of neighboring 2 TADs.

3 A) Schematic describing TAD boundary insulation alteration events. A TAD boundary loss (left)

4 is associated with a strong increase in inter-TAD interactions of two adjacent T cell TADs and

5 loss of CTCF binding. A TAD boundary gain (right) is associated with a strong decrease in inter-

6 TAD interactions of two adjacent T-ALL TADs and concomitant gain of CTCF binding.

7 B) Total numbers of TAD boundary gains / losses identified between T-ALL and T cells before 8 integrating differential CTCF binding information. All pairwise comparisons of $\mathrm{T}$ cells act as 9 negative controls.

$10 \mathbf{C}+\mathbf{D})$ Representation of TAD insulation alteration events (red dots) among all pairs of adjacent 11 TADs (black dots). Plots depict comparisons for TAD boundary losses of adjacent T cell TADs 12 within T-ALL samples (C left), or between $\mathrm{T}$ cell samples 1 and 3 (C right). Plots in D) depict 13 comparisons for TAD boundary gains of adjacent T-ALL TADs when compared to $\mathrm{T}$ cell 14 samples (D left), or between $\mathrm{T}$ cell samples 1 and 3 (D right). Encircled adjacent TADs 15 demarcate gain / loss of insulation accompanied by more than one gained / lost CTCF binding, 16 respectively. Significant changes in CTCF binding were calculated using the R package DiffBind 17 and filtered for FDR $<0.1$ and $\log 2$ fold-change $>1 /<-1$.

$18 \mathbf{E}+\mathbf{F})$ All TAD boundary alterations (boundary loss $(\mathrm{E})$, boundary gain $(\mathrm{F})$ ) from comparisons in 19 C) and D) between T-ALL and T cells were used to estimate heterogeneity in insulation changes 20 of such boundary alterations across all analyzed $\mathrm{Hi}-\mathrm{C}$ samples. Hic-ratio insulation scores for 21 each boundary and sample were compared vs. the average hic-ratio insulation score of all $\mathrm{T}$ cell 22 samples. Boundary losses $(n=78)$ come with a decrease in insulation scores on average, while 23 boundary gains $(n=83)$ come with increase in insulation scores across all T-ALLs on average 24 when compared to the average hic-ratio insulation score of all $\mathrm{T}$ cell samples.

Figure 4: MYC overexpression in leukemia is associated with CTCF loss and TAD 27 boundary insulation loss.

28 A) Hi-C interaction heatmaps (first row) showing the MYC locus. Second row shows heatmaps 29 of per-bin $\log 2$ fold-change interactions when compared to T cell 1. In T cells, MYC is located 30 in the upstream TAD at its upstream boundary, while the super-enhancer cluster is located in the 31 downstream TAD. 
1 B) CTCF and H3K27ac ChIP-Seq tracks for the MYC locus. CTCF orientation is shown for 2 canonical CTCF binding motifs derived from PWMScan ${ }^{66}$ (database JASPAR CORE 3 vertebrates; filtered by $\mathrm{p}$-value $<1 \mathrm{E}-5)$. Green boxes on the left and right highlight CTCF

4 binding in the upstream and downstream TAD boundaries, with the downstream boundary 5 showing multiple CTCF binding sites with the same orientation. Red box in the middle 6 highlights loss of CTCF insulation in leukemia. Grey box towards the right highlights the super7 enhancer cluster. ChIP-Seq tracks show fold-enrichment over input where applicable, counts8 per-million reads otherwise. ATAC-Seq tracks show counts-per-million reads.

9 C) 4C-Seq analysis using $M Y C$ promoter as viewpoint. Positive y-axis shows interactions with

10 the MYC promoter viewpoint as normalized read counts, while negative $\mathrm{y}$-axis shows 11 significance of differential interactions between $\mathrm{T}$ cells and CUTLL1 as $\log (\mathrm{p}$-value) derived 12 using edgeR function glmQLFTest. The three grey boxes highlight three areas of strong 13 H3K27ac signal within the super-enhancer element (N-Me/NDME, CEE, BDME/BENC) that 14 correlate with $M Y C$ promoter interactions. Tracks below show H3K27ac ChIP-Seq tracks for T 15 cells and CUTLL1 as fold-enrichment over input.

16 D) MYC expression determined by RNA-Seq and shown as log2 FPKM for T cells and T-ALL 17 samples. Statistical evaluation was performed using two-sided edgeR analysis followed by 18 multiple testing correction.

19 E) Distance between $M Y C$ promoter and center enhancer element (MYC-CCE) measured by 20 DNA-FISH analysis (left). Statistical difference between distributions of probe distances was 21 calculated using two-sample one-sided Kolmogorov Smirnov test following the hypothesis of 22 increased probe-distance in T cells when compared to T-ALL. Probe-pairs T cells = 993; Probe23 pairs CUTLL1 $=$ 2001. Median distance $\mathrm{T}$ cells $=412.84 \mu \mathrm{m}$. Median distance CUTLL1 = $24264.28 \mu \mathrm{m}$.

Figure 5: NOTCH1 inhibition affects promoter-enhancer looping specifically of NOTCH127 dependent enhancers

28 A) Differential H3K27ac occupancy analysis based on H3K27ac ChIP-Seq in CUTLL1 with and 29 without NOTCH1-inhibitor $\gamma \mathrm{SI}$. The three identified groups consist of stable non-promoter 30 H3K27ac signal (middle, black, $n=2949$ ), increased (upper, pink, $n=125$ ) and reduced non31 promoter H3K27ac signal (lower, light-blue, $n=243$ ). Heatmap shows the H3K27ac signal as 
1 fold-enrichment over input within $+/-2.5 \mathrm{~kb}$ around the summit of the identified $\mathrm{H} 3 \mathrm{~K} 27 \mathrm{ac}$ peaks

2 and line plots depict quantification of H3K27ac signal within these regions, both created with

3 DeepTools ${ }^{67}$. Differential analysis was performed with the $\mathrm{R}$ package DiffBind and differential

4 peaks were selected using FDR $<0.05, \log 2$ fold-change $>1.0$ or $<-1.0$.

5 B) Overlap of constant, increased and reduced H3K27ac peaks with NOTCH1-dynamic sites 6 previously defined by ${ }^{21}$. Quantification of H3K27ac signal shown as fold-enrichment over input

7 (right panel) specifically for peaks with reduced H3K27ac signal and dynamic NOTCH1 binding

8 (n=76). Statistical evaluation was performed using two-sided Fisher test against all non-coding

9 H3K27ac peaks overlapping dynamic NOTCH1 binding.

10 C) Changes in chromatin interactions upon $\gamma \mathrm{SI}$ between non-promoter H3K27ac peaks of 11 interest defined in A) and B) and nearby gene promoters (defined using H3K27ac HiChIP data in

12 CUTLL1) are shown as $\log 2$ fold-change of normalized interaction score. Each dot represents

13 such a promoter-enhancer interaction defined by H3K27ac HiChIP. Significance of global shifts

14 compared to enhancer-promoter loops of stable enhancers (grey, left) is calculated by unpaired

15 one-tailored t-test, following the hypothesis of a positive correlation between enhancer activity 16 and promoter-looping.

17 D) Changes in gene expression upon $\gamma \mathrm{SI}$ for all genes defined in C) are shown as $\log 2$ fold-

18 change of FPKM calculated from GRO-Seq data. Significance of global differences when

19 compared to genes associated with stable H3K27ac signal is calculated by unpaired one-tailored

20 t-test, following the hypothesis of a positive correlation between promoter-enhancer looping and

21 gene expression.

22 E+F) 4C-Seq analysis using LUNARl promoter (E) or APCDDI enhancer (F) as viewpoints.

23 Positive y-axis shows interactions with the viewpoint as normalized read counts, while negative

$24 \mathrm{y}$-axis shows significance of differential interactions between untreated and $\gamma$ SI treated CUTLL1

25 as $\log (\mathrm{p}$-value) calculated using edgeR function glmQLFTest. The grey box in E) shows a

26 previously reported intronic IGF1R enhancer; the grey box in F) shows the APCDD1 promoter.

27 Tracks below show H3K27ac and NOTCH1 ChIP-Seq and GRO-Seq (positive strand only)

28 before and after $\gamma \mathrm{SI}$ treatment as fold-enrichment over input where applicable, counts-per-

29 million otherwise. 
1 Figure 6: CDK7 inhibition concomitantly reduces H3K27ac levels and associated 2 promoter-enhancer looping

3 A) LOLA analysis result for the overlap between public ChIP-Seq data in Jurkat from the LOLA

4 database with $\gamma$ SI-insensitive and $\gamma$ SI-sensitive enhancers. Statistical differences in overlap

5 between $\gamma$ SI-insensitive and sensitive enhancers with ChIP-Seq peaks were calculated using a

6 two-sided Fisher exact test.

7 B) Differential H3K27ac occupancy analysis based on H3K27ac ChIP-Seq in CUTLL1 treated 8 with either DMSO or THZ1. The three identified groups consist of stable non-promoter 9 H3K27ac signal (middle, white, $n=1396$ ), increased (upper, grey, $n=2246$ ) and reduced non10 promoter H3K27ac signal (lower, pink, $n=3248$ ). Heatmap shows the H3K27ac signal as fold11 enrichment over input within $+/-2.5 \mathrm{~kb}$ around the summit of the identified H3K27ac peaks and 12 line plots depict quantification of $\mathrm{H} 3 \mathrm{~K} 27 \mathrm{ac}$ signal within these regions. Differential analysis was 13 performed with the R package DiffBind and differential peaks were selected using FDR $<0.05$, $14 \log 2$ fold-change $>1.0$.

15 C) Hi-C integration with $\mathrm{H} 3 \mathrm{~K} 27 \mathrm{ac}$ peaks of interest. Changes in chromatin interactions upon 16 THZ1 between non-promoter H3K27ac peaks of interest defined in B) and nearby gene 17 promoters (defined using H3K27ac HiChIP data in CUTLL1) are shown as log2 fold-change of 18 normalized interaction score. Each dot represents such a promoter-enhancer interaction.

19 Significance of global shifts compared to enhancer-promoter interactions associated with stable 20 enhancers (grey, left) is calculated by an unpaired one-sided t-test, following the hypothesis of a 21 positive correlation between enhancer activity and promoter-looping.

22 D+E) $4 \mathrm{C}$-Seq analysis using $M Y C$ promoter (D) or $I K Z F 2$ promoter (E) as viewpoints. Positive $23 \mathrm{y}$-axis shows interactions with the viewpoint as normalized read counts, while negative y-axis 24 shows significance of differential interactions between untreated and THZ1 treated CUTLL1 as $25 \log (\mathrm{p}$-value) calculated using edgeR function glmQLFTest. The grey boxes show the enhancer 26 elements. Tracks below show H3K27ac before and after THZ1 treatment and CDK7 ChIP-Seq 27 track from Jurkat cell line, and represent fold-enrichment over input where applicable and 28 counts-per-million reads otherwise. 


\section{Supplementary Figure 1:}

2 A) Read alignment statistics for Hi-C datasets generated within this study, as absolute reads (left)

3 and relative reads (in \%, right). "ds.accepted.intra" are all intra-chromosomal reads used for all

4 downstream analyses.

5 B) Genome-wide stratum-adjusted correlation coefficient (SCC) scores for all pair-wise 6 comparisons of the three T cell, five canonical primary T-ALL samples, two T-ALL cell lines 7 and four ETP-ALL Hi-C datasets. HiCRep was used to calculate chromosome-wide correlation 8 scores, which were averaged across all chromosomes for each pair-wise comparison. The 9 HiCRep smoothing parameter $\mathrm{X}$ was set to 1.0 .

10 C) Principal Component Analysis (PCA) of the genome-wide compartment scores for each Hi-C 11 dataset, separating normal from leukemic samples on PC1.

12 D) Compartment shifts identified between $\mathrm{T}$ cells, T-ALL and ETP-ALL. Assignment of A 13 compartment was done using an average c-score > 0.1 in either all T cell, T-ALL or ETP-ALL 14 samples as well as higher enrichment in H3K27ac signal and vice versa B compartment for 15 average c-score $<-0.1$. Significance for differences between pairwise comparisons of T cells, T16 ALL and ETP-ALL was determined using a two-sided t-test between c-scores, and compartment 17 shifts were determined using p-value $<0.1$.

19 Supplementary Figure 2:

20 A) Copy number variants (CNVs) determined from $\mathrm{Hi}-\mathrm{C}$ for each sample individually using 21 HiCnv software ${ }^{36}$. CNV gain was determined if the average estimated copy-number was $>3.5$, 22 and CNV loss was determined if the average estimated copy-number was $<1.25$. Default setting 23 of chromosome 2 was used as reference chromosome for copy-number estimation.

24 B) Average genomic area of TADs (in percent) of differential / stable activity or all TADs 25 overlapping with compartment shifts. Differentially active / stable TADs are defined in Figure 26 2A; compartment shifts are defined in Figure 1E.

27 C+D) WGS detected CNVs (gain / loss) and tandem duplication from overlap with differentially 28 active TADs (C) / boundaries of differentially active TADs (D), both defined in Figure 2A, 29 showed no overlap. Overlap was performed using bedtools intersect, using 1bp overlap between 30 TAD area (C) / TAD boundary extended by 40kb on each side (D) and CNV/tandem duplication. 
1 E) Integration of SNVs detected from WGS of T-ALL 1 (left) and T-ALL 2 (right) with TAD

2 activity results. SNVs per Megabase $(\mathrm{Mb})$ were counted within genomic areas of all, stably or

3 differentially active TADs. Statistical analysis was performed using two-sided Fisher exact test

4 between numbers of all SNVs overlapping loss/gain of TAD activity and numbers of all SNVs

5 within stably active TADs.

6 F) Integration of SNVs with CTCF binding motifs within differential CTCF binding genome-

7 wide. Differential CTCF binding between all profiled T-ALL and T cell samples was determined

8 using DiffBind (FDR $<0.1$, $\log 2$ fold-change $>1$ for increased CTCF binding and $\log 2$ fold-

9 change < -1 for decreased CTCF binding in T-ALL; stable CTCF was determined by $\log 2$ fold-

10 change $>-0.2$ and $\log 2$ fold-change $<0.2$ ). Statistical analysis was performed using two-sided

11 Fisher exact test between overlap of SNVs with differential CTCF binding and overlap of SNVs

12 with stable CTCF binding.

13 G) Integration of SNVs with CTCF binding motifs within differential CTCF binding that overlap 14 with TAD boundaries of differentially / stably active TADs. Differential CTCF binding between

15 all profiled T-ALL and T cell samples was determined using DiffBind (FDR $<0.1, \log 2$ fold-

16 change $>1$ for increased CTCF binding and $\log 2$ fold-change $<-1$ for decreased CTCF binding

17 in T-ALL; stable CTCF was determined by $\log 2$ fold-change $>-0.2$ and $\log 2$ fold-change $<0.2$ ).

18 Statistical analysis was performed using two-sided Fisher exact test between overlap of SNVs

19 with differential CTCF binding and overlap of SNVs with stable CTCF binding.

\section{Supplementary Figure 3:}

22 A) Hi-C interaction heatmaps (first row) showing the IKZF2 locus (black circle). Second row 23 shows heatmaps of $\log 2$ fold-change interactions when compared to T cell 1.

24 B) $\mathrm{H} 3 \mathrm{~K} 27 \mathrm{ac}$ ChIP-Seq tracks for IKZF2 locus in T cells and CUTLL1, NOTCH1 ChIP-Seq 25 tracks for CUTLL1. Tracks represent fold-enrichment over input where applicable and counts26 per-million reads otherwise.

27 C) Quantifications for intra-TAD activity (left; as highlighted in A)) and expression of IKZF2 28 (right). Statistical evaluation for intra-TAD activity was performed using paired two-sided t-test 29 of average per interaction-bin for IKZF2 TAD between T cells and T-ALL, followed by multiple 30 testing correction (see methods). IKZF2 expression was determined by RNA-Seq and shown as 
$1 \quad \log 2$ counts per million (CPM) for $\mathrm{T}$ cells and T-ALL samples; statistical evaluation was

2 performed using edgeR followed by multiple testing correction.

3 D) Hi-C interaction heatmaps (first row) showing the CYLD locus (black circle). Second row

4 shows heatmaps of $\log 2$ fold-change interactions when compared to T-cell 1.

5 E) H3K27ac ChIP-Seq tracks for CYLD locus in T cells and CUTLL1, NOTCH1 ChIP-Seq

6 tracks for CUTLL1. Tracks represent fold-enrichment over input where applicable and counts-

7 per-million reads otherwise.

8 F) Quantifications for intra-TAD activity (left; as highlighted in D)) and expression of CYLD

9 (right). Statistical evaluation for intra-TAD activity was performed using paired two-sided t-test

10 of average per interaction-bin for CYLD TAD between T cells and T-ALL, followed by multiple

11 testing correction (see methods). CYLD expression was determined by RNA-Seq and shown as

$12 \log 2$ counts per million (CPM) for $\mathrm{T}$ cells and T-ALL samples; statistical evaluation was

13 performed using edgeR followed by multiple testing correction.

\section{Supplementary Figure 4:}

16 A) Comparisons of intra-TAD activity between T cells, T-ALL and ETP-ALL samples.

17 B) Overlap of differentially active TADs between the two comparisons of T cells vs T-ALL and

18 T cells vs ETP-ALL, visualized as venn diagram. Red and blue colors correspond to differences

19 as highlighted in A).

20 C+D) Integration of RNA-Seq (minimum per-gene expression filter FPKM > 1) within TADs 21 with decreased / increased intra-TAD activity for ETP-ALL vs T cells (C) and ETP-ALL vs T-

22 ALL (D). For each such gene, the respective $\log 2$ fold-change in expression between ETP-ALL 23 and T cells (C) / T-ALL and ETP-ALL (D) taken from RNA-Seq is shown. Significant global 24 differences are calculated by an unpaired one-sided t-test comparing genes from TADs with 25 decreased / increased intra-TAD activity with genes from stable TADs, following the hypothesis 26 of a positive correlation between expression and intra-TAD activity changes.

\section{Supplementary Figure 5:}

29 A+B) Overlap of TAD boundaries detected as altered in insulation capacity as in Figures $3 \mathrm{C}$ and $303 \mathrm{D}$ with genomic inversions (A) or insertions/deletions (indels) (B) from WGS of T-ALL 1 (top) 31 and T-ALL 2 (bottom). Overlap was determined by bedtools intersect, using a 1bp overlap. 


\section{Supplementary Figure 6:}

2 A) CTCF ChIP-qPCR of the CTCF binding site in the lost MYC TAD boundary, shown as fold-

3 enrichment over input. Significant differences compared to $\mathrm{T}$ cells were calculated with an

4 unpaired one-sided t-test, following the hypothesis of loss of CTCF binding in T-ALL samples

5 as determined from the genome-wide analysis ( $n=3$ replicates for T cells, T-ALL 1, T-ALL 2,

6 CUTLL1 and Jurkat; $n=2$ replicates for T-ALL 3 and T-ALL 4).

7 B) Targeted sanger sequencing indicates no mutation in T-ALL in the motif of CTCF binding

8 site. Tracks show chromatogram of individual base calls (left). Whole genome sequencing

9 indicates no mutation in T-ALL in the motif of CTCF binding site. Tracks show percent (mis-

10 )matches compared to reference sequence in all reads covering the respective genomic position

11 (right).

12 C) CTCF ChIP-qPCR before and after treatment with global DNA-demethylation agent 5-

13 azacytidine. Statistical significance was determined using two-sided t-test ( $n=2$ replicates).

14 D) ATAC-Seq quantification for $\mathrm{T}$ cells and Jurkat for the genomic area covering loss of CTCF

15 binding in the downstream TAD boundary of MYC. Data was normalized to the average T cell

16 signal, shown in percent ( $n=3$ replicates). Statistical evaluation was performed using DiffBind,

17 following multiple testing correction.

19 Supplementary Figure 7:

20 A) 4C-Seq analysis using $M Y C$ promoter as viewpoint. Positive y-axis shows interactions with

21 the MYC promoter viewpoint as normalized read counts, while negative $\mathrm{y}$-axis shows

22 significance of differential interactions between $\mathrm{T}$ cells and primary T-ALL samples as $\log (\mathrm{p}-$

23 value). The three grey boxes highlight three areas of strong H3K27ac signal within the super-

24 enhancer element (N-Me/NDME, CEE, BDME/BENC) that correlate with strong $M Y C$ promoter

25 interactions. H3K27ac ChIP-Seq tracks for T cells and CUTLL1 are represented below as fold-

26 enrichment over input.

28 Supplementary Figure 8:

29 A) Schematic of Cas9+Synthetic guide transfection of activated T cells. 
1 B) Sequence showing CTCF motif in the insulator region in $\mathrm{T}$ cells targeted for CRISPR-based 2 deletion. sgRNA targeting sequence within the CTCF motif is highlighted. Sequencing of

3 sgRNA target site indicates various indels along with frequencies observed for each indel.

4 C) CTCF ChIP-qPCR validation of reduced CTCF binding in edited $\mathrm{T}$ cells compared to 5 unedited T cells ( $n=2$ replicates). Statistical significance was determined using unpaired one6 sided t-test following the hypothesis that mutation/deletion of the CTCF binding site would lead 7 to abrogation of CTCF binding.

8 D) qPCR comparing MYC expression in edited T cells compared to unedited T cells $(n=3$

9 replicates). Statistical significance was determined using unpaired two-sided t-test.

10 E) 4C-Seq analysis using $M Y C$ promoter as viewpoint in edited and unedited T cells. Positive y11 axis shows interactions with the viewpoint as normalized read counts, while negative y-axis 12 shows significance of differential interactions between the two samples as $\log (\mathrm{p}$-value) 13 calculated with edgeR function glmQLFTest ( $n=2$ replicates). Tracks below show CTCF ChIP14 Seq track from CUTLL1 and H3K27ac ChIP-Seq tracks for naïve T cells and CUTLL1 as fold15 enrichment over input.

\section{Supplementary Figure 9:}

18 A) Volcano plot showing differential intra-TAD activity for the comparison of CUTLL1 treated

19 with either DMSO or $\gamma$ SI. TAD activity changes are highlighted for log 2 fold-change of average 20 activity $>0.58 /<-0.58$ and with FDR $<0.05$. Statistical evaluation was performed using paired 21 two-sided t-test between all per bin-interactions for the same TAD between DMSO and $\gamma \mathrm{SI}$ 22 treated cells.

23 B) Representation of TAD boundary alteration events (red dots; none identified) among all pairs 24 of adjacent TADs (black dots). Plots depict pair-wise comparisons for TAD boundary losses of 25 adjacent CUTLL1 (untreated, left) TADs and for TAD boundary gains of adjacent CUTLL1 $(\gamma$ SI 26 treated, right) TADs. However, in this analysis, no single TAD boundary alteration was 27 identified reaching the same outlier threshold as the leukemia vs. normal comparison (red dotted 28 lines; taken from Figure 3 C) and D)).

29 C) Virtual 4C representation of H3K27ac HiChIP in CUTLL1, using MYC promoter as 30 viewpoint (chr8: 128,747,680), showing edgeR-normalized counts-per-million (CPM). H3K27ac 
1 ChIP-Seq track for MYC locus in CUTLL1, shown as fold-enrichment over input. Detected

2 significant loops as arc-representation (below) from mango pipeline ${ }^{68}$ (FDR $<0.1$; CPM $>5$ ).

3 D) Quantification of H3K27ac signal (enrichment over input) by ChIP-Seq (left), chromatin

4 interaction of the highest peak by 4C-Seq (center) for the interaction of LUNAR1 promoter with

5 its upstream enhancer element and LUNAR1 expression by GRO-Seq (right). All quantifications

6 are normalized to the respective average $\mathrm{T}$ cell signal, shown in percent. Significance of

7 differences was calculated using diffBind (for H3K27ac ChIP-Seq, FDR) and edgeR (for 4C-Seq

8 interactions and GRO-Seq as p-value and FDR respectively).

9 E) Quantification of H3K27ac signal by ChIP-Seq (left), chromatin interaction of the highest 10 peak by 4C-Seq (center) for the interaction of $A P C D D 1$ enhancer with the downstream APCDD1

11 promoter and APCDD1 expression by GRO-Seq (right). All quantifications are normalized to the

12 respective average $\mathrm{T}$ cell signal, shown in percent. Significance of differences was calculated

13 using diffBind (for H3K27ac ChIP-Seq, FDR) and edgeR (for 4C-Seq interactions and GRO-Seq

14 as p-value and FDR respectively). Error bars indicate standard deviation.

15 F) Schematic of $\gamma$ SI sensitive and insensitive enhancer.

16 G) Comparison of the width of two classes of identified H3K27ac peaks. All peaks are 17 overlapping dynamic NOTCH1 sites, and are of either stable H3K27ac signal (black, $n=111$ ) or 18 decreased signal (green, $n=76$ ) as defined in Figure 5A. Significant difference between the 19 distributions is estimated by a two-sided Wilcoxon test.

21 Supplementary Figure 10:

22 A) 4C-Seq analysis using $M Y C$ promoter as viewpoint after $\gamma \mathrm{SI}$ treatment. Positive y-axis shows 23 interactions with the viewpoint as normalized read counts, while negative y-axis shows 24 significance of differential interactions between untreated and $\gamma$ SI treated CUTLL1 as $\log (\mathrm{p}-$ 25 value) calculated using edgeR function glmQLFTest ( $n=5$ for CUTLL1 DMSO; $n=3$ for 26 CUTLL1 $\gamma \mathrm{SI}$ ). The grey boxes highlight enhancer elements N-Me/NDME, CEE and 27 BDME/BENC. Tracks below show H3K27ac, NOTCH1 ChIP-Seq and GRO-Seq (positive 28 strand only) tracks before and after $\gamma \mathrm{SI}$ treatment as fold-enrichment where applicable, and 29 counts-per-million reads otherwise.

30 B) Quantification of changes in $\mathrm{H} 3 \mathrm{~K} 27 \mathrm{ac}$ signal (enrichment over input), chromatin interactions 31 of the highest peaks by 4C-Seq for the interactions of MYC with respective super-enhancer 
1 elements and MYC expression after $\gamma \mathrm{SI}$. H3K27ac signal quantification is specific for $\mathrm{N}$ -

$2 \mathrm{Me} / \mathrm{NDME}, \mathrm{CEE}$ and BDME/BENC. Interaction changes are measured by centering the 40kb

3 bin on highest peaks within N-Me/NDME, CEE or BDME/BENC elements. MYC expression

4 after $\gamma \mathrm{SI}$ treatment was measured by qPCR. All quantifications are normalized to the respective

5 average T cell signal, shown in percent. Significance is shown as false-discovery rate (FDR) for

6 H3K72ac signal change (R package DiffBind), p-value for chromatin interaction change (edgeR

7 function glmQLFTest) or one-tailored t-test for qPCR changes.

8 C) Western blot analysis of CUTLL1 cells treated with either DMSO or $\gamma$ SI and immunoblotted 9 with MYC antibody.

10 D) $4 \mathrm{C}$-Seq analysis using $I K Z F 2$ promoter as viewpoint after $\gamma \mathrm{SI}$ treatment. Positive y-axis 11 shows normalized interaction strength with the viewpoint, while negative $y$-axis shows 12 significance of differential interactions between untreated and $\gamma \mathrm{SI}$ treated CUTLL1 as $\log (\mathrm{p}-$ 13 value) calculated using edgeR function glmQLFTest ( $n=5$ for CUTLL1 DMSO; $n=3$ for 14 CUTLL1 $\gamma$ SI). The grey boxes highlight IKZF2 enhancer element identified by HiChIP analysis.

15 Tracks below show H3K27ac, NOTCH1 ChIP-Seq and GRO-Seq (negative strand only) tracks

16 before and after $\gamma$ SI treatment as fold-enrichment over input where applicable, and counts-per17 million reads otherwise.

18 E) Quantification of changes in H3K27ac signal (enrichment over input), chromatin interactions 19 and IKZF2 expression after $\gamma \mathrm{SI}$. H3K27ac signal quantification is specific for enhancer 20 highlighted in D). Interaction changes are measured by centering the $40 \mathrm{~kb}$ bin on the highest 21 enhancer peak. IKZF2 expression after $\gamma$ SI treatment was measured by GRO-Seq. All 22 quantifications are normalized to the respective average $\mathrm{T}$ cell signal, shown in percent. 23 Significance is shown as false-discovery rate (FDR) for H3K72ac signal change (R package 24 DiffBind), p-value for chromatin interaction change (edgeR function glmQLFTest) or one25 tailored t-test for qPCR changes.

26 F) CTCF ChIP-qPCR of lost MYC boundary in DMSO or $\gamma$ SI treated CUTLL1 cells $(n=3)$.

27 Significance was calculated using unpaired two-sided t-test. 


\section{Supplementary Figure 11:}

2 A) Quantification of changes in $\mathrm{H} 3 \mathrm{~K} 27 \mathrm{ac}$ signal (enrichment over input), chromatin interactions 3 and MYC expression after THZ1. H3K27ac signal quantification is specific for N-Me/NDME,

$4 \mathrm{CEE}$ and BDME/BENC. Interaction changes are measured by centering the $40 \mathrm{~kb}$ bin on highest 5 peaks within N-Me/NDME, CEE or BDME/BENC elements. MYC expression after

6 THZ1 treatment was measured by qPCR. All quantifications are normalized to the respective 7 average CUTLL1 DMSO signal, shown in percent. Significance is shown as false-discovery rate 8 (FDR) for H3K72ac signal change (R package DiffBind), p-value for chromatin interaction 9 change (edgeR function glmQLFTest) or two-sided t-test for qPCR changes.

10 B) Western blot analysis of CUTLL1 cells treated with either DMSO or THZ1 and 11 immunoblotted with MYC antibody.

12 C) CTCF ChIP-qPCR, shown as enrichment over input, of CTCF site in lost boundary in MYC 13 locus in CUTLL1 cells treated with either DMSO or THZ1 ( $n=3)$. Significance was calculated 14 using unpaired two-sided t-test.

15 D) Inter-probe distance between $M Y C$ promoter and center enhancer element (MYC-CCE) 16 measured by DNA-FISH analysis in CUTLL1 cells treated with either DMSO or THZ1.

17 Statistical difference between distributions of probe distances was calculated using two-sample

18 one-sided Kolmogorov Smirnov test following the hypothesis of increased probe-distance in 19 CUTLL1 cells treated with THZ1 when compared to DMSO. Representative FISH image of 20 CUTLL1 cells treated with either DMSO or THZ1 (right). Probe-pairs CUTLL1 DMSO = 2001.

21 Probe-pairs CUTLL1 THZ1 = 1308. Median distance CUTLL1 DMSO = 264.28 $\mu$ m. Median 22 distance CUTLL1 THZ1 $=321.69 \mu \mathrm{m}$.

23 E) 4C-Seq analysis using $M Y C$ promoter as viewpoint in Jurkat cells. Positive y-axis shows 24 normalized interaction strength with the viewpoint, while negative y-axis shows significance of 25 differential interactions between untreated and THZ1 treated Jurkat cells as $\log (\mathrm{p}$-value) 26 calculated using edgeR function glmQLFTest $(n=3)$.

27 F) Quantification of changes in chromatin interactions of $M Y C$ enhancers after THZ1 treatment 28 in Jurkat. Interaction changes are measured by centering the 40kb bin on N-Me/NDME, CEE or 29 the BDME/BENC. Significance is shown as p-value for chromatin interaction changes between 30 DMSO and THZ1 treated cells (edgeR function glmQLFTest). 
1 G) Quantification of changes in $\mathrm{H} 3 \mathrm{~K} 27 \mathrm{ac}$ signal (enrichment over input) and chromatin 2 interactions of IKZF2 enhancer after THZ1 treatment in CUTLL1. All quantifications are

3 normalized to the respective average Jurkat DMSO signal, shown in percent. Significance is

4 shown as p-value for chromatin interaction change (edgeR function glmQLFTest).

6 SUPPLEMENTAL TABLE LEGENDS

7 Supplementary Table 1:

8 References and accession numbers (where applicable) for public Hi-C, ChIP-Seq and RNA-Seq

9 datasets integrated in this study.

10 Supplementary Table 2:

11 Read alignment statistics for all Hi-C, 4C-Seq, ChIP-Seq, RNA-Seq and GRO-Seq datasets 12 generated within this study.

13 Supplementary Table 3:

14 List of known driver mutations identified in the primary T-ALL cohort.

\section{Supplementary Table 4:}

16 Number of TAD calls per sample using hic-ratio TAD caller ${ }^{27}$ at $40 \mathrm{~kb}$ Hi-C matrix resolution 17 and $500 \mathrm{~kb}$ insulation-window.

\section{REFERENCES}

201 Lupiáñez, Darío G. et al. Disruptions of Topological Chromatin Domains Cause

21 Pathogenic Rewiring of Gene-Enhancer Interactions. Cell 161, 1012-1025,

22 doi:10.1016/j.cell.2015.04.004.

232 Flavahan, W. A. et al. Insulator dysfunction and oncogene activation in IDH mutant

24 gliomas. Nature 529, 110-114, doi:10.1038/nature16490 (2016).

253 Spielmann, M., Lupianez, D. G. \& Mundlos, S. Structural variation in the 3D genome.

26 Nat Rev Genet, doi:10.1038/s41576-018-0007-0 (2018).

274 Dekker, J. \& Mirny, L. The 3D Genome as Moderator of Chromosomal Communication.

$28 \quad$ Cell 164, 1110-1121, doi:10.1016/j.cell.2016.02.007 (2016).

295 Lieberman-Aiden, E. et al. Comprehensive mapping of long-range interactions reveals

30 folding principles of the human genome. Science (New York, N.Y.) 326,

31 doi:10.1126/science.1181369 (2009). 
16 Dixon, J. R. et al. Topological domains in mammalian genomes identified by analysis of 2 chromatin interactions. Nature 485, doi:10.1038/nature11082 (2012).

37 Nora, E. P. et al. Spatial partitioning of the regulatory landscape of the X-inactivation

$4 \quad$ centre. 485,381 , doi:10.1038/nature11049

5 https://www.nature.com/articles/nature11049\#supplementary-information (2012).

68 Hnisz, D. et al. Super-enhancers in the control of cell identity and disease. Cell 155, 9347 947, doi:10.1016/j.cell.2013.09.053 (2013).

89 Whyte, W. A. et al. Master transcription factors and mediator establish super-enhancers $9 \quad$ at key cell identity genes. Cell 153, 307-319, doi:10.1016/j.cell.2013.03.035 (2013).

1010 Gong, Y. et al. Stratification of TAD boundaries reveals preferential insulation of super-

11 enhancers by strong boundaries. Nature communications 9, 542, doi:10.1038/s41467-

12 018-03017-1 (2018).

1311 Ong, C. T. \& Corces, V. G. CTCF: an architectural protein bridging genome topology

14 and function. Nat Rev Genet 15, 234-246, doi:10.1038/nrg3663 (2014).

1512 Hnisz, D., Day, D. S. \& Young, R. A. Insulated Neighborhoods: Structural and

16 Functional Units of Mammalian Gene Control. Cell 167, 1188-1200,

17 doi:10.1016/j.cell.2016.10.024 (2016).

1813 Nora, E. P. et al. Targeted Degradation of CTCF Decouples Local Insulation of 19 Chromosome Domains from Genomic Compartmentalization. Cell 169, 930-944.e922, 20 doi:10.1016/j.cell.2017.05.004.

2114 Rao, S. S. P. et al. Cohesin Loss Eliminates All Loop Domains. Cell 171, 305-320.e324, 22 doi:10.1016/j.cell.2017.09.026 (2017).

2315 Guo, Y. et al. CRISPR Inversion of CTCF Sites Alters Genome Topology and 24 Enhancer/Promoter Function. Cell 162, 900-910, doi:10.1016/j.cell.2015.07.038 (2015).

2516 Narendra, V. et al. CTCF establishes discrete functional chromatin domains at the Hox 26 clusters during differentiation. Science (New York, N.Y.) 347, 1017-1021, 27 doi:10.1126/science.1262088 (2015).

2817 Weischenfeldt, J. et al. Pan-cancer analysis of somatic copy-number alterations 29 implicates IRS4 and IGF2 in enhancer hijacking. Nat Genet 49, 65-74, 30 doi:10.1038/ng.3722 (2017). 
118 Petrovic, J. et al. Oncogenic Notch Promotes Long-Range Regulatory Interactions within

2 Hyperconnected 3D Cliques. Molecular cell 73, 1174-1190.e1112,

3 doi:10.1016/j.molcel.2019.01.006 (2019).

419 Grabher, C., von Boehmer, H. \& Look, A. T. Notch 1 activation in the molecular

5 pathogenesis of T-cell acute lymphoblastic leukaemia. Nature reviews. Cancer 6, 347-

6 359, doi:10.1038/nrc1880 (2006).

720 Belver, L. \& Ferrando, A. The genetics and mechanisms of T cell acute lymphoblastic

8 leukaemia. Nature reviews. Cancer 16, 494-507, doi:10.1038/nrc.2016.63 (2016).

921 Wang, H. et al. NOTCH1-RBPJ complexes drive target gene expression through

10 dynamic interactions with superenhancers. Proceedings of the National Academy of

11 Sciences 111, 705-710, doi:10.1073/pnas.1315023111 (2014).

1222 Hunger, S. P. \& Mullighan, C. G. Acute Lymphoblastic Leukemia in Children. New

13 England Journal of Medicine 373, 1541-1552, doi:10.1056/NEJMra1400972 (2015).

1423 Weng, A. P. et al. Activating mutations of NOTCH1 in human T cell acute lymphoblastic

15 leukemia. Science (New York, N.Y.) 306, 269-271, doi:10.1126/science.1102160 (2004).

1624 Liu, Y. et al. The genomic landscape of pediatric and young adult T-lineage acute

17 lymphoblastic leukemia. 49, 1211, doi:10.1038/ng.3909

18 https://www.nature.com/articles/ng.3909\#supplementary-information (2017).

1925 Palomero, T. et al. CUTLL1, a novel human T-cell lymphoma cell line with t(7;9)

20 rearrangement, aberrant NOTCH1 activation and high sensitivity to gamma-secretase

21 inhibitors. Leukemia 20, 1279-1287, doi:10.1038/sj.leu.2404258 (2006).

2226 Sulis, M. L. et al. NOTCH1 extracellular juxtamembrane expansion mutations in T-ALL.

23 Blood 112, 733-740, doi:10.1182/blood-2007-12-130096 (2008).

2427 Lazaris, C., Kelly, S., Ntziachristos, P., Aifantis, I. \& Tsirigos, A. HiC-bench:

25 comprehensive and reproducible $\mathrm{Hi}-\mathrm{C}$ data analysis designed for parameter exploration

26 and benchmarking. BMC Genomics 18, 22, doi:10.1186/s12864-016-3387-6 (2017).

2728 Zhang, J. et al. The genetic basis of early T-cell precursor acute lymphoblastic

$28 \quad$ leukaemia. 481, 157, doi:10.1038/nature10725

29 https://www.nature.com/articles/nature10725\#supplementary-information (2012). 
129 Kourtis, N. et al. Oncogenic hijacking of the stress response machinery in T cell acute

2 lymphoblastic leukemia. Nat Med 24, 1157-1166, doi:10.1038/s41591-018-0105-8

3 (2018).

430 Chen, L. et al. Genetic drivers of epigenetic and transcriptional variation in human

$5 \quad$ immune cells. Cell 167, 1398-1414. e1324 (2016).

631 Shochat, C. et al. Gain-of-function mutations in interleukin-7 receptor-alpha (IL7R) in

7 childhood acute lymphoblastic leukemias. The Journal of experimental medicine 208,

8 901-908, doi:10.1084/jem.20110580 (2011).

932 Zenatti, P. P. et al. Oncogenic IL7R gain-of-function mutations in childhood T-cell acute

10 lymphoblastic leukemia. Nat Genet 43, 932-939, doi:10.1038/ng.924 (2011).

1133 Gutierrez, A. et al. High frequency of PTEN, PI3K, and AKT abnormalities in T-cell

12 acute lymphoblastic leukemia. Blood 114, 647-650, doi:10.1182/blood-2009-02-206722

13 (2009).

1434 Yang, T. et al. HiCRep: assessing the reproducibility of Hi-C data using a stratum-

15 adjusted correlation coefficient. Genome Research 27, 1939-1949,

16 doi:10.1101/gr.220640.117 (2017).

1735 Zheng, X. \& Zheng, Y. CscoreTool: fast Hi-C compartment analysis at high resolution.

18 Bioinformatics 34, 1568-1570, doi:10.1093/bioinformatics/btx802 (2018).

1936 Chakraborty, A. \& Ay, F. Identification of copy number variations and translocations in 20 cancer cells from Hi-C data. Bioinformatics, doi:10.1093/bioinformatics/btx664 (2017).

2137 Zhang, Z. et al. Expression of a non-DNA-binding isoform of Helios induces T-cell

22 lymphoma in mice. Blood 109, 2190-2197, doi:10.1182/blood-2005-01-031930 (2007).

2338 Vilimas, T. et al. Targeting the NF-kappaB signaling pathway in Notch1-induced T-cell

24 leukemia. Nat Med 13, 70-77, doi:10.1038/nm1524 (2007).

2539 Espinosa, L. et al. The Notch/Hes1 pathway sustains NF-kappaB activation through

26 CYLD repression in T cell leukemia. Cancer Cell 18, 268-281,

27 doi:10.1016/j.ccr.2010.08.006 (2010).

2840 de Wit, E. et al. CTCF Binding Polarity Determines Chromatin Looping. Molecular cell 29 60, 676-684, doi:10.1016/j.molcel.2015.09.023 (2015). 
141 Weng, A. P. et al. c-Myc is an important direct target of Notch1 in T-cell acute

2 lymphoblastic leukemia/lymphoma. Genes Dev 20, 2096-2109, doi:10.1101/gad.1450406

3 (2006).

442 Palomero, T. et al. NOTCH1 directly regulates c-MYC and activates a feed-forward-loop

5 transcriptional network promoting leukemic cell growth. Proceedings of the National

6 Academy of Sciences of the United States of America 103, 18261-18266,

7 doi:10.1073/pnas.0606108103 (2006).

843 Herranz, D. et al. A NOTCH1-driven MYC enhancer promotes T cell development,

9 transformation and acute lymphoblastic leukemia. Nat Med 20, 1130-1137,

10 doi: $10.1038 / \mathrm{nm} .3665$

11 http://www.nature.com/nm/journal/v20/n10/abs/nm.3665.html\#supplementary-information

12 (2014).

1344 Yashiro-Ohtani, Y. et al. Long-range enhancer activity determines Myc sensitivity to

14 Notch inhibitors in T cell leukemia. Proceedings of the National Academy of Sciences of

15 the United States of America 111, E4946-4953, doi:10.1073/pnas.1407079111 (2014).

1645 Shi, J. et al. Role of SWI/SNF in acute leukemia maintenance and enhancer-mediated

17 Myc regulation. Genes \& Development 27, 2648-2662, doi:10.1101/gad.232710.113

18 (2013).

1946 Bahr, C. et al. A Myc enhancer cluster regulates normal and leukaemic haematopoietic

20 stem cell hierarchies. Nature 553, 515-520, doi:10.1038/nature25193 (2018).

2147 Deangelo, D. J. et al. A phase I clinical trial of the notch inhibitor MK-0752 in patients

22 with T-cell acute lymphoblastic leukemia/lymphoma (T-ALL) and other leukemias.

23 Journal of Clinical Oncology 24, 6585-6585, doi:10.1200/jco.2006.24.18_suppl.6585

24 (2006).

2548 Mumbach, M. R. et al. HiChIP: efficient and sensitive analysis of protein-directed

26 genome architecture. Nature methods 13, 919, doi:10.1038/nmeth.3999

27 https://www.nature.com/articles/nmeth.3999\#supplementary-information (2016).

2849 Delmore, Jake E. et al. BET Bromodomain Inhibition as a Therapeutic Strategy to Target 29 c-Myc. Cell 146, 904-917, doi:10.1016/j.cell.2011.08.017 (2011). 
150 Trimarchi, T. et al. Genome-wide Mapping and Characterization of Notch-Regulated

2 Long Noncoding RNAs in Acute Leukemia. Cell 158, 593-606,

3 doi:10.1016/j.cell.2014.05.049.

451 Medyouf, H. et al. High-level IGF1R expression is required for leukemia-initiating cell

5 activity in T-ALL and is supported by Notch signaling. The Journal of experimental medicine 208, 1809-1822, doi:10.1084/jem.20110121 (2011).

52 Sheffield, N. C. \& Bock, C. LOLA: enrichment analysis for genomic region sets and regulatory elements in R and Bioconductor. Bioinformatics 32, 587-589 (2015).

Kwiatkowski, N. et al. Targeting transcription regulation in cancer with a covalent CDK7 inhibitor. Nature 511, 616-620, doi:10.1038/nature13393 (2014).

1455 Chipumuro, E. et al. CDK7 Inhibition Suppresses Super-Enhancer-Linked Oncogenic Transcription in MYCN-Driven Cancer. Cell 159, 1126-1139, doi:https://doi.org/10.1016/j.cell.2014.10.024 (2014). with a covalent CDK7 inhibitor. Cancer cell 26, 909-922, doi:10.1016/j.ccell.2014.10.019 (2014).

57 Northcott, P. A. et al. Enhancer hijacking activates GFI1 family oncogenes in medulloblastoma. Nature 511, 428-434, doi:10.1038/nature13379 (2014).

Hnisz, D. et al. Activation of proto-oncogenes by disruption of chromosome neighborhoods. Science (New York, N.Y.) 351, 1454-1458, doi:10.1126/science.aad9024 (2016).

3061 Bonev, B. et al. Multiscale 3D Genome Rewiring during Mouse Neural Development. Cell 171, 557-572.e524, doi:10.1016/j.cell.2017.09.043 (2017). 
162 Dixon, J. R. et al. Chromatin architecture reorganization during stem cell differentiation.

$2 \quad$ Nature 518, 331-336, doi:10.1038/nature14222 (2015).

363 Donaldson-Collier, M. C. et al. EZH2 oncogenic mutations drive epigenetic,

4 transcriptional, and structural changes within chromatin domains. Nature Genetics 51,

5 517-528, doi:10.1038/s41588-018-0338-y (2019).

664 Bellavia, D., Palermo, R., Felli, M. P., Screpanti, I. \& Checquolo, S. Notch signaling as a

7 therapeutic target for acute lymphoblastic leukemia. Expert Opinion on Therapeutic

$8 \quad$ Targets 22, 331-342, doi:10.1080/14728222.2018.1451840 (2018).

965 Siersbaek, R. et al. Transcription factor cooperativity in early adipogenic hotspots and

10 super-enhancers. Cell Rep 7, 1443-1455, doi:10.1016/j.celrep.2014.04.042 (2014).

1166 Ambrosini, G., Groux, R. \& Bucher, P. PWMScan: a fast tool for scanning entire

12 genomes with a position-specific weight matrix. Bioinformatics 34, 2483-2484,

13 doi:10.1093/bioinformatics/bty127 (2018).

1467 Ramirez, F. et al. deepTools2: a next generation web server for deep-sequencing data 15 analysis. Nucleic Acids Res 44, W160-165, doi:10.1093/nar/gkw257 (2016).

1668 Phanstiel, D. H., Boyle, A. P., Heidari, N. \& Snyder, M. P. Mango: a bias-correcting

17 ChIA-PET analysis pipeline. Bioinformatics 31, 3092-3098,

18 doi:10.1093/bioinformatics/btv336 (2015). 
Figure 1

A

B

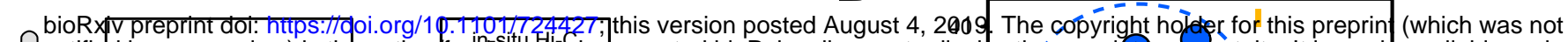

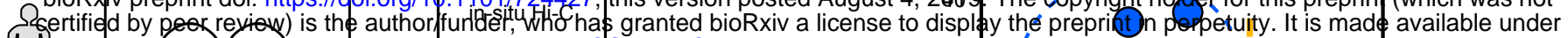
oncogene

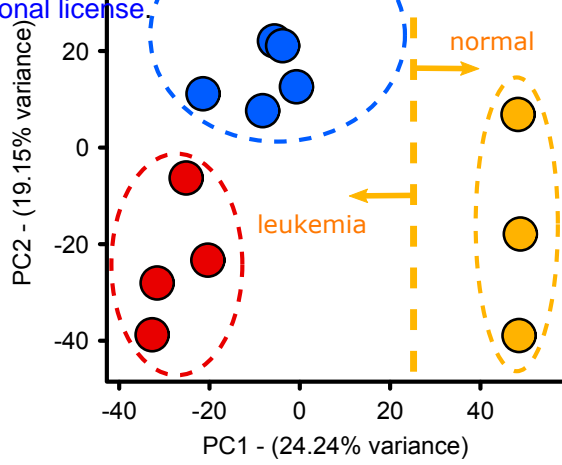

$\square$ Cluster 1

$\square$ Cluster 2

$\square$ Cluster 3

C

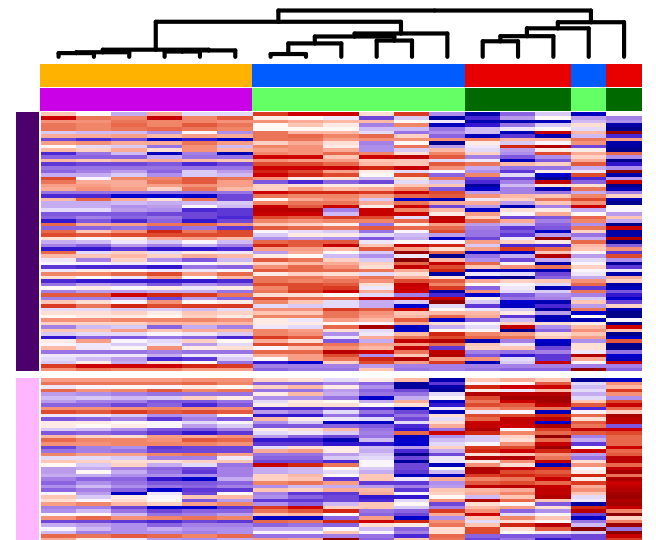

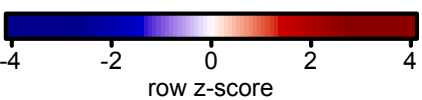

$\square$ Cluster 1 $\square$ Cluster 2

$\square$ Cluster 3

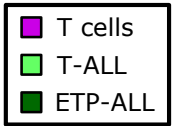

T-ALL signature $\square$ ETP-ALL signature

D

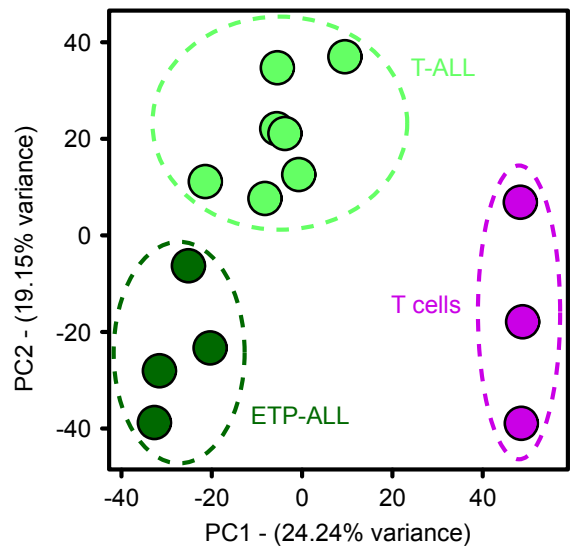

E
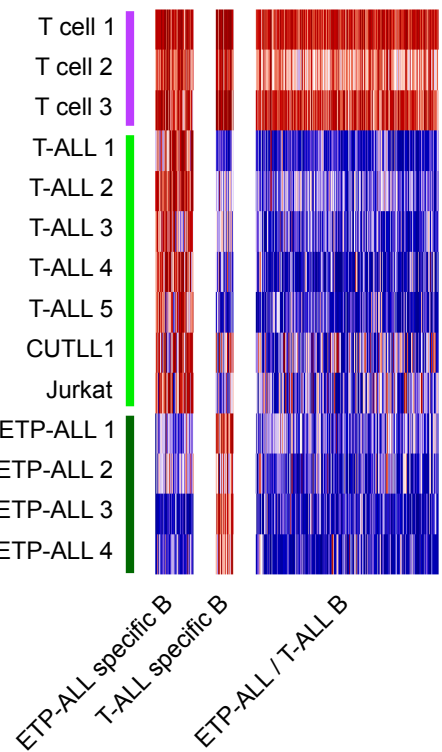
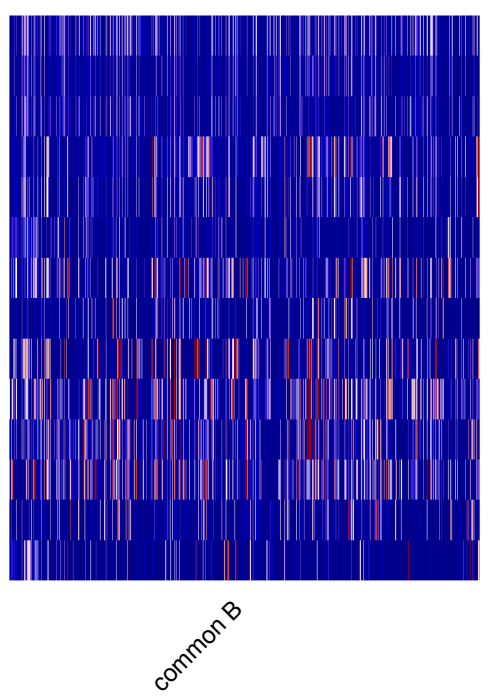
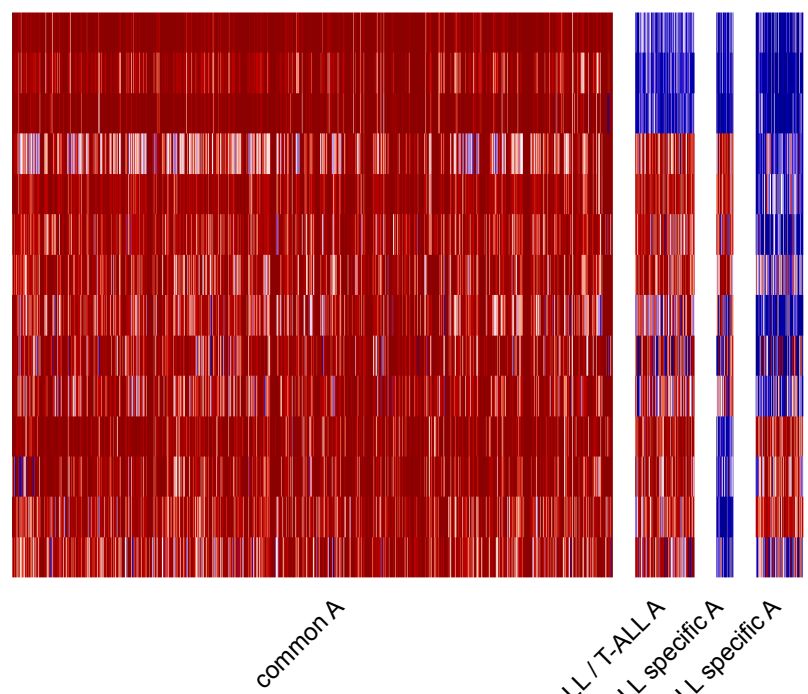

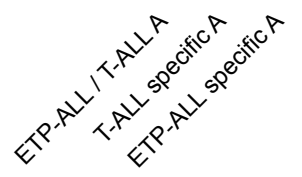




\section{Figure 2}

A

B

T cells vs T-ALLs

T cell 1 vs T cell 3

C
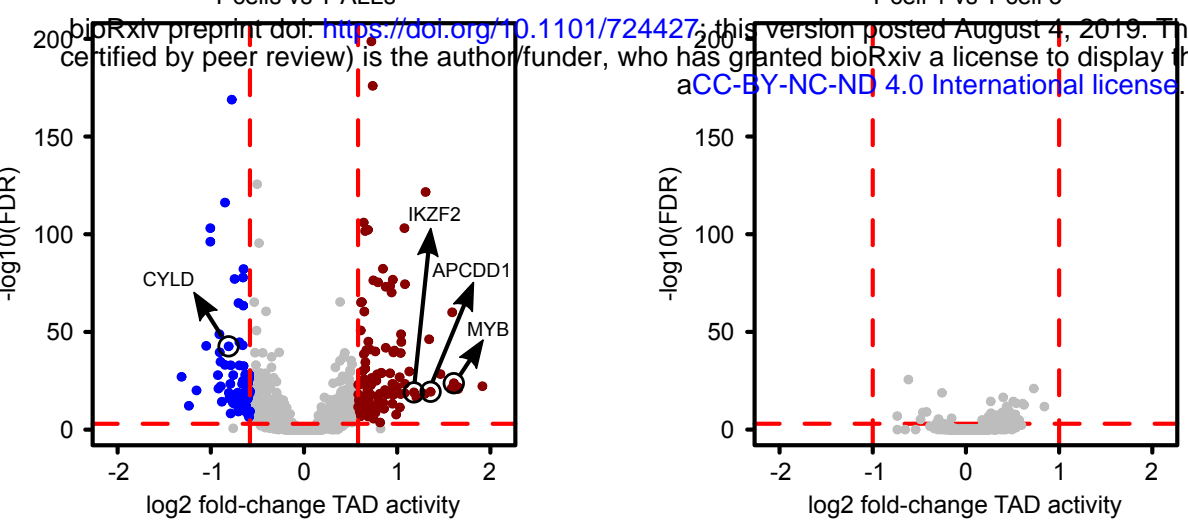

D

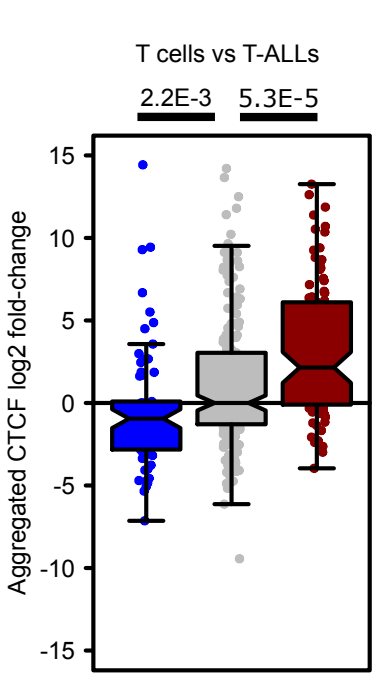

E

$\mathbf{F}$

T cells T-ALL

holder for this preprint (which was not perpetuity. It is made availablo tinder
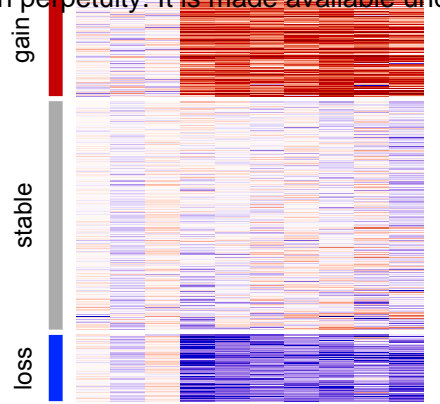

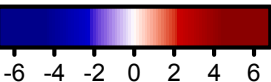

log2 fold-change of

TAD activity vs avg. T cells
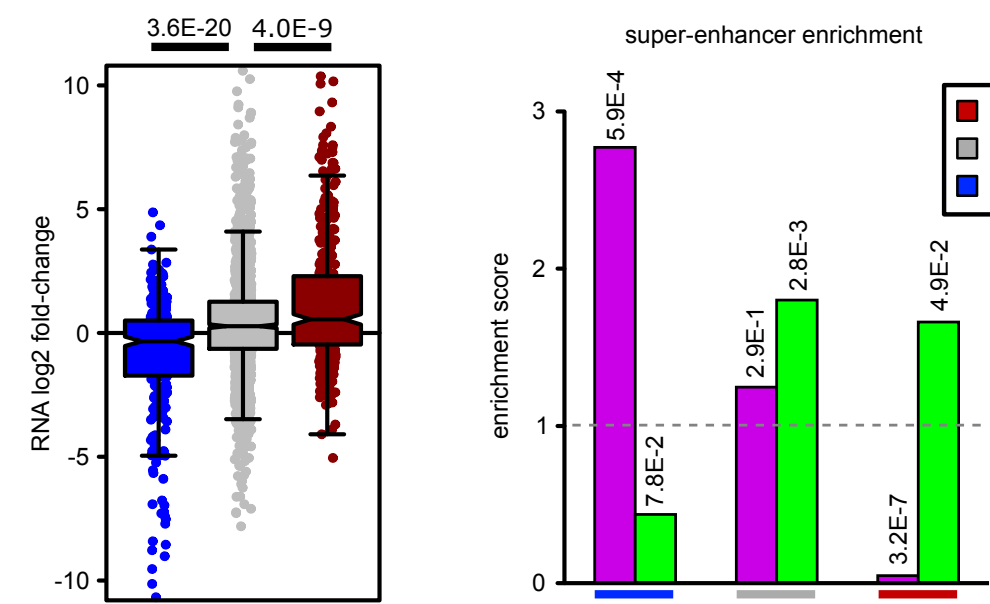

$\square$ Gain in TAD activity in T-ALL

$\square$ Stable TAD activity

$\square$ Loss in TAD activity in T-ALL

G

T cell 3

T cell 1

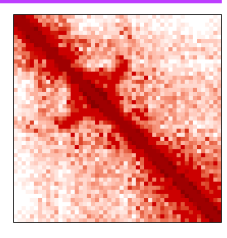

$\bigvee^{-19 C D D 1}$

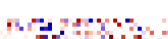

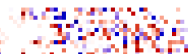

at:

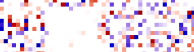

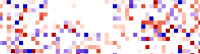

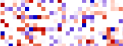

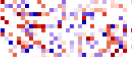

T-ALL 1

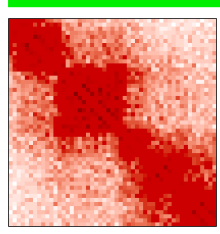

tistrits

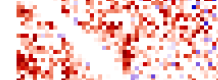

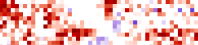

$2+2$

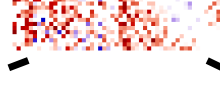

if $2, y$

$H$
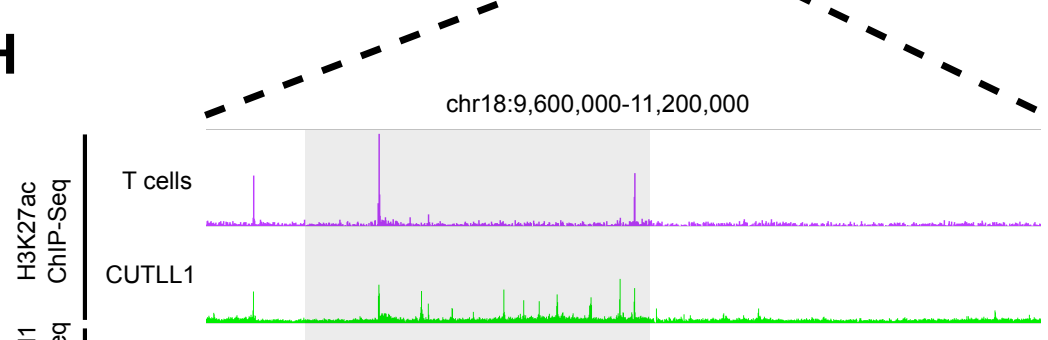

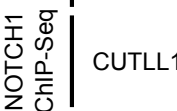

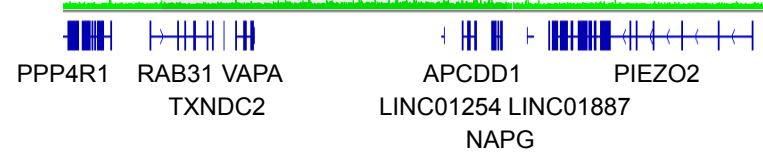

chr18:9,500,000-11,500,000

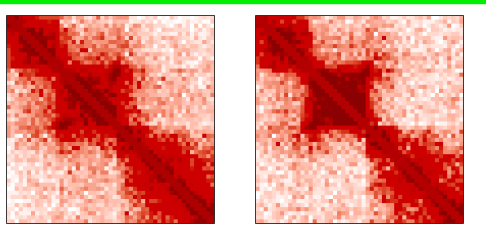

T-ALL 4

CUTLL1
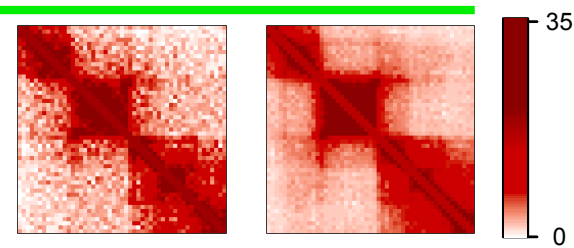

h:4astor 3.0

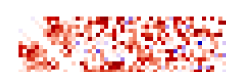

74253

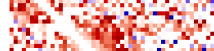

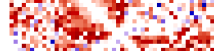

$74 \mathrm{Cw}^{2}$

72,40
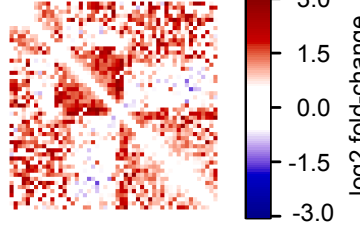

3.8E-9
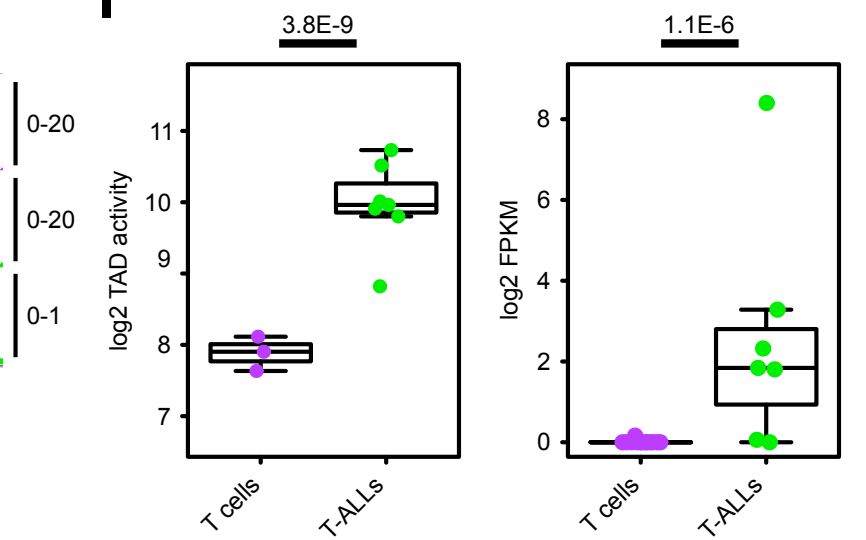


\section{Figure 3}

A

TAD boundary loss

TAD boundary gain

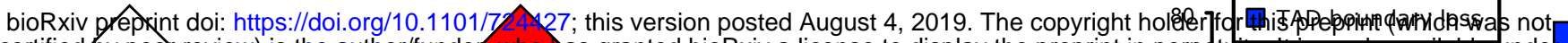
certifieoby peex review) is the author/funde, who has granted bioRxiv a license to display the preprint in perpequity. It is made availabte unde
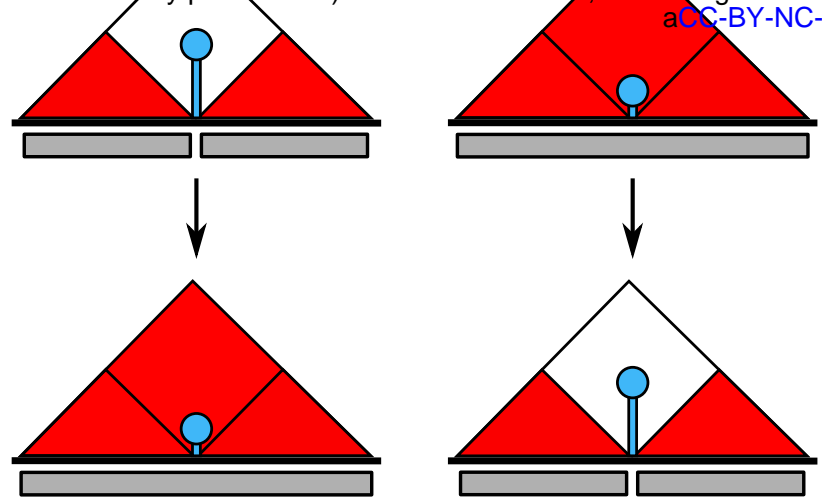

high interaction frequency

low interaction frequency

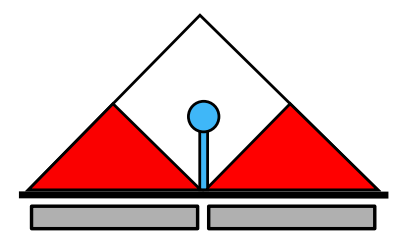

weak CTCF binding

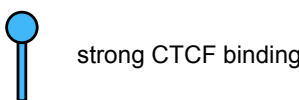

$\square$ TAD

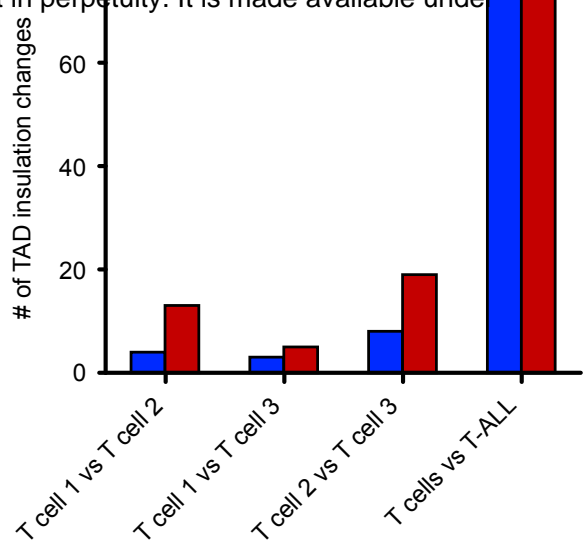

C

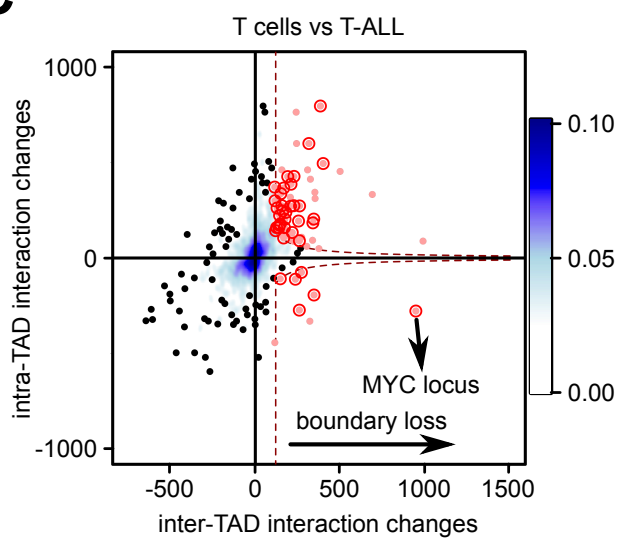

D

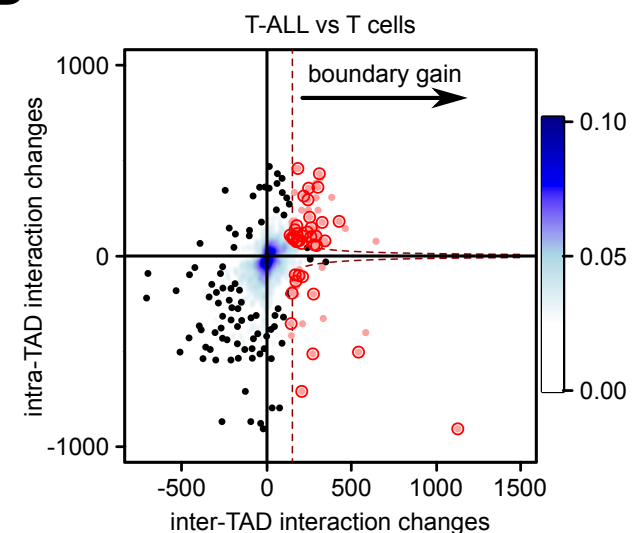

- TAD pair

Significant outlier

- Significant outlier w/ CTCF change

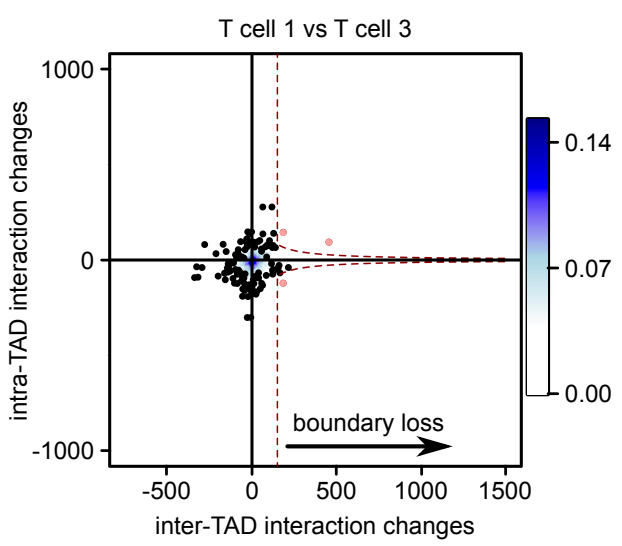

E

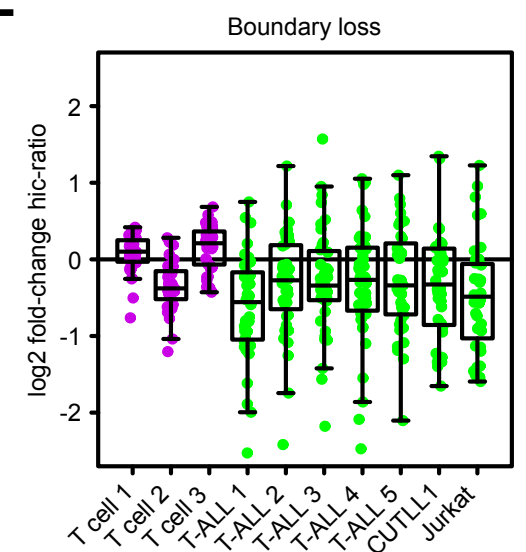

F

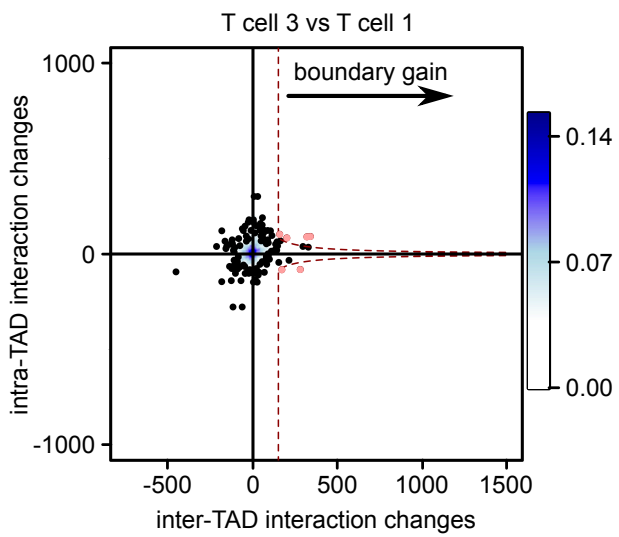

- TAD pair

Significant outlier

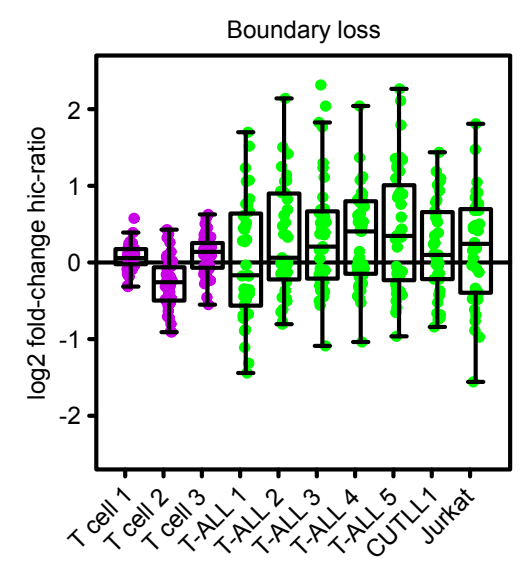

$\square$ T cells T-ALL 


\section{Figure 4}

A

chr8:128,000,000-131,500,000

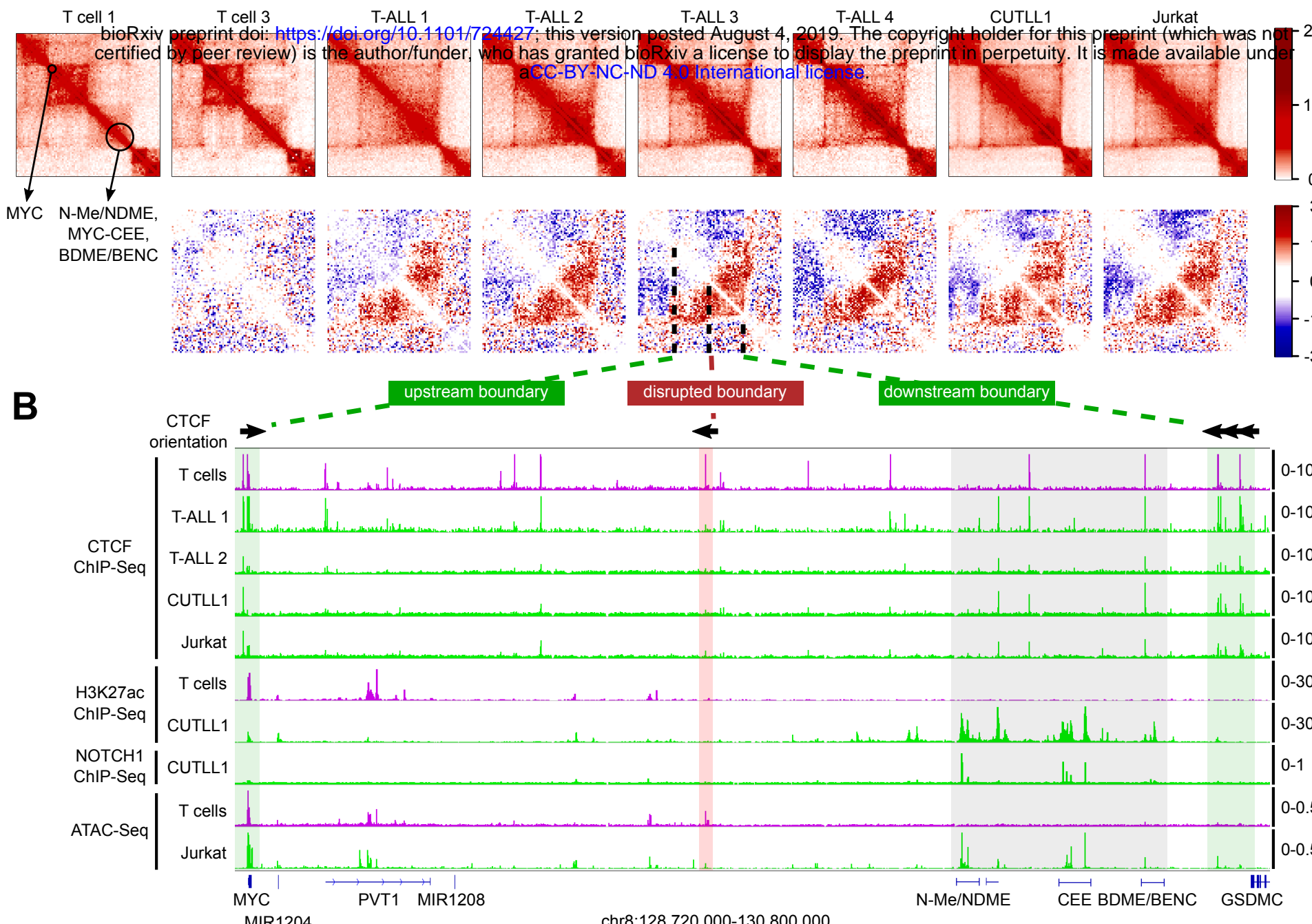

C
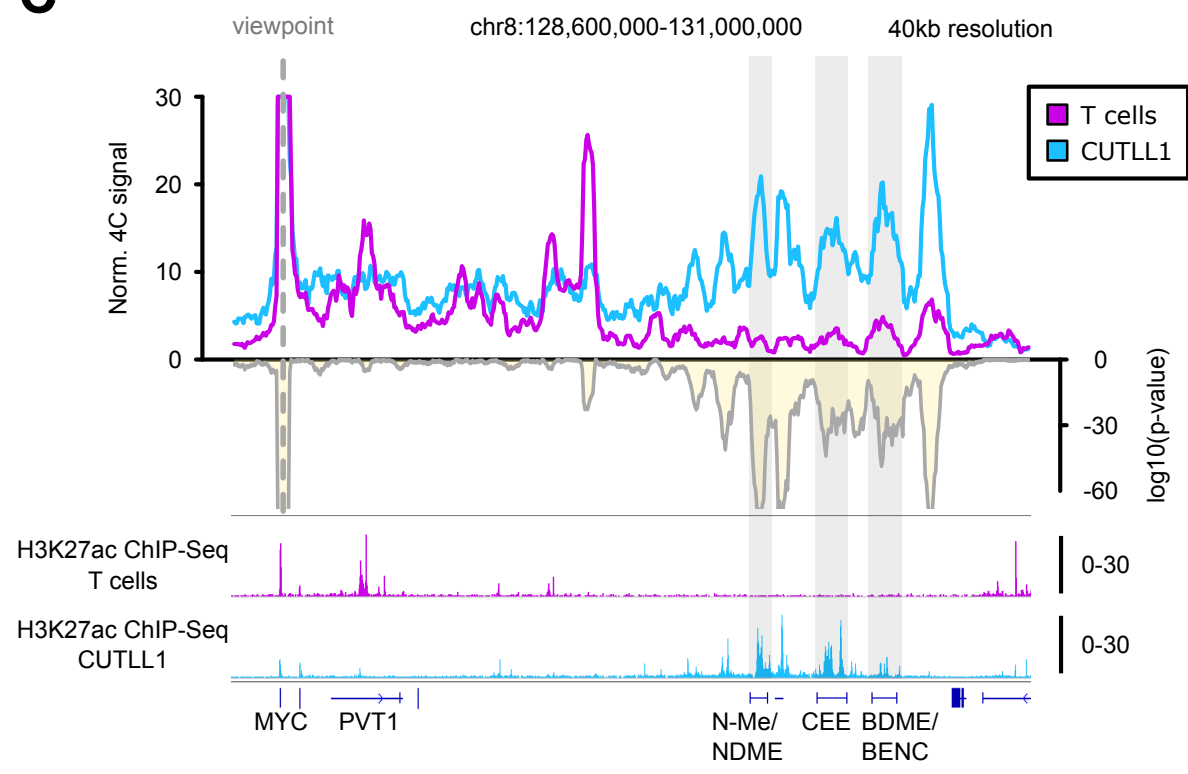

D

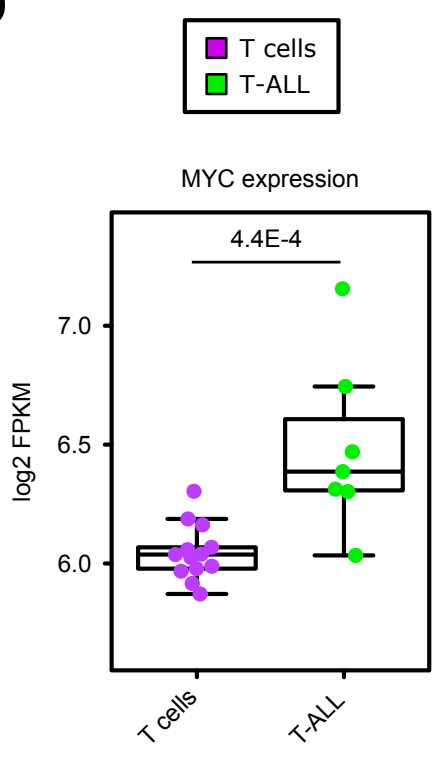

E

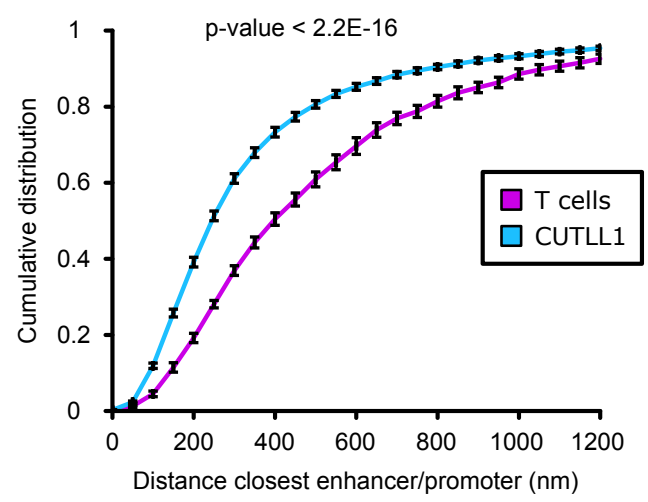

T cells
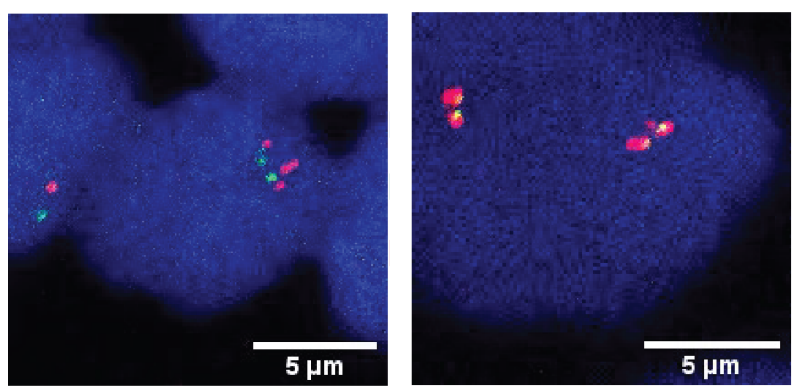

O MYC promoter
O MYC-CEE
O DAPI 


\section{Figure 5}

A CUTLL1 DMSO CUTLL1 YSI

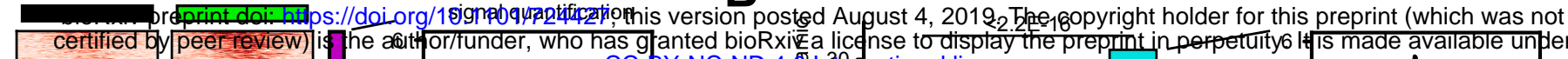
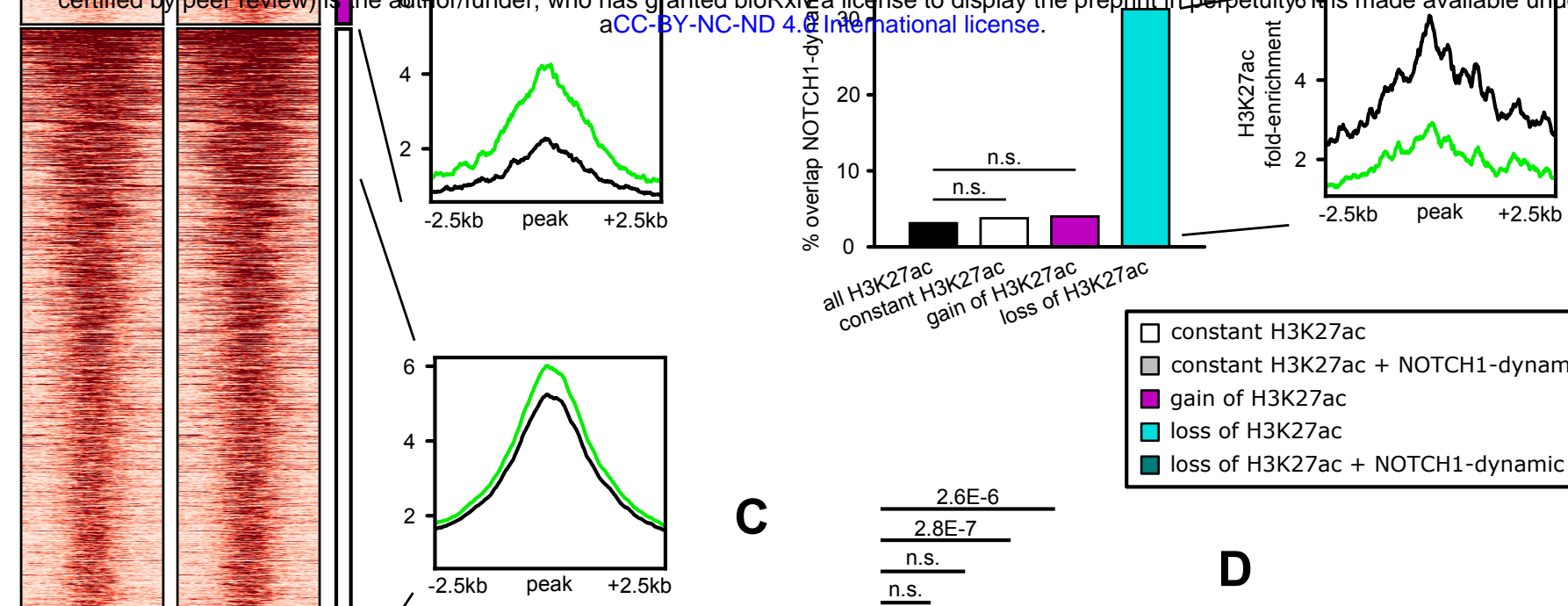

all $\mathrm{H} 3 \mathrm{~K} 27 \mathrm{aC}$
constant $\mathrm{H} 3 \mathrm{~K} 27 \mathrm{aC}$ of $\mathrm{H} 3 \mathrm{~K} 27 \mathrm{aC}$
gain of $\mathrm{H} 3 \mathrm{~K} 27 \mathrm{aC}$

$\square$ constant H3K27ac
$\square$ constant H3K27ac + NOTCH1-dynamic
$\square$ gain of H3K27ac
$\square$ loss of H3K27ac
$\square$ loss of H3K27ac + NOTCH1-dynamic

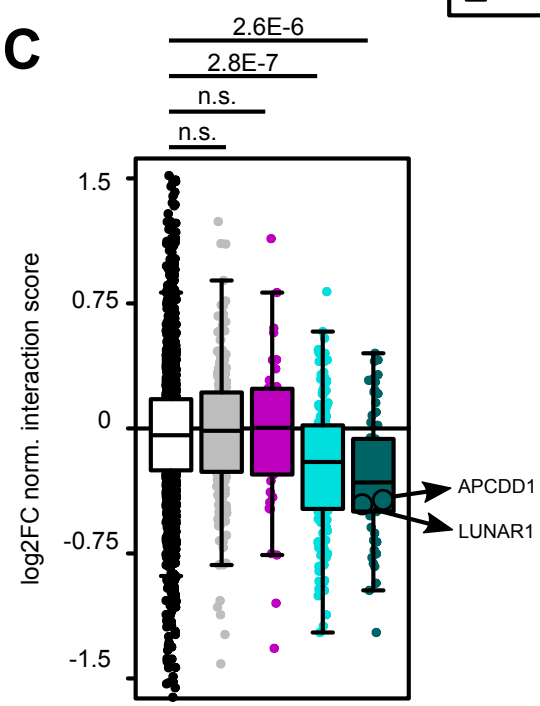

D
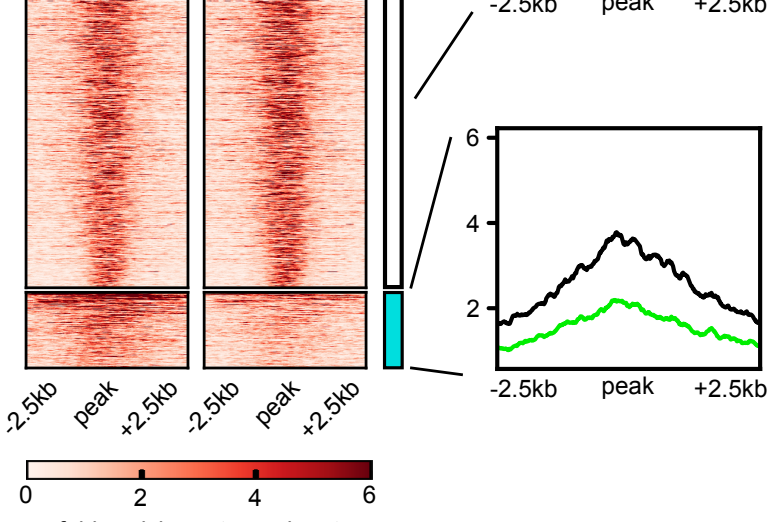

E

chr15:99,363,000-99,613,000

F

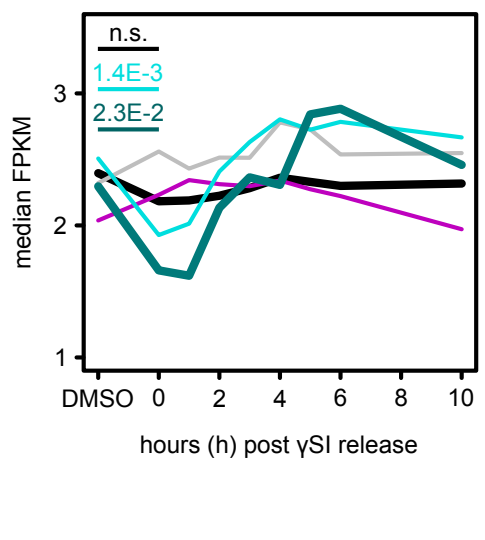

iewpoint

$10 \mathrm{~kb}$ resolution
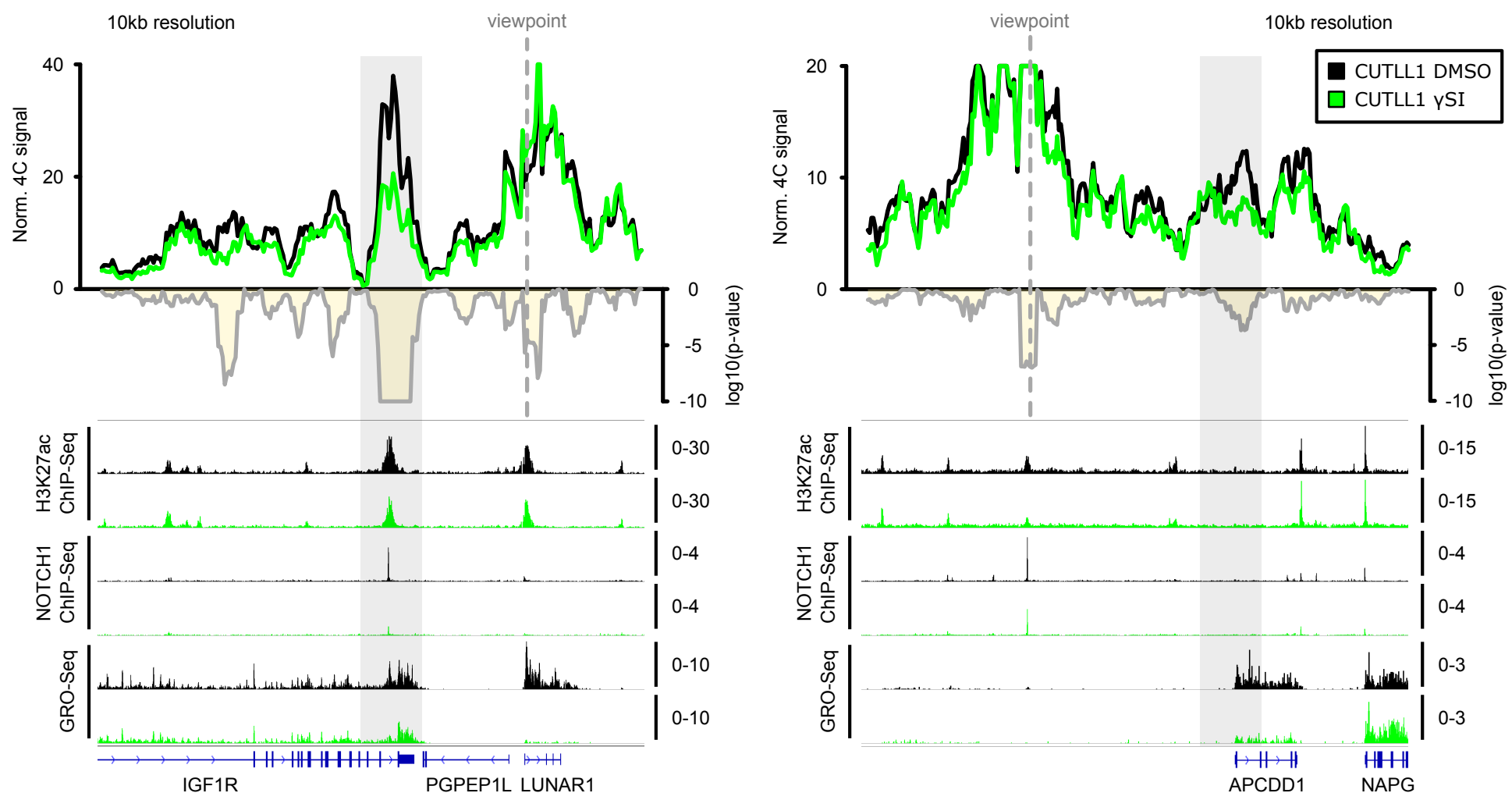

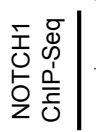

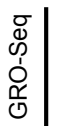

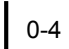

0-4

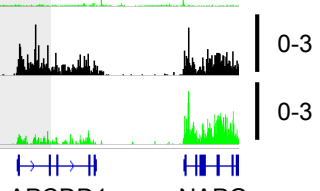


Figure 6

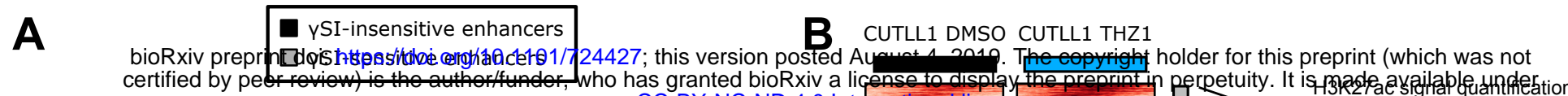

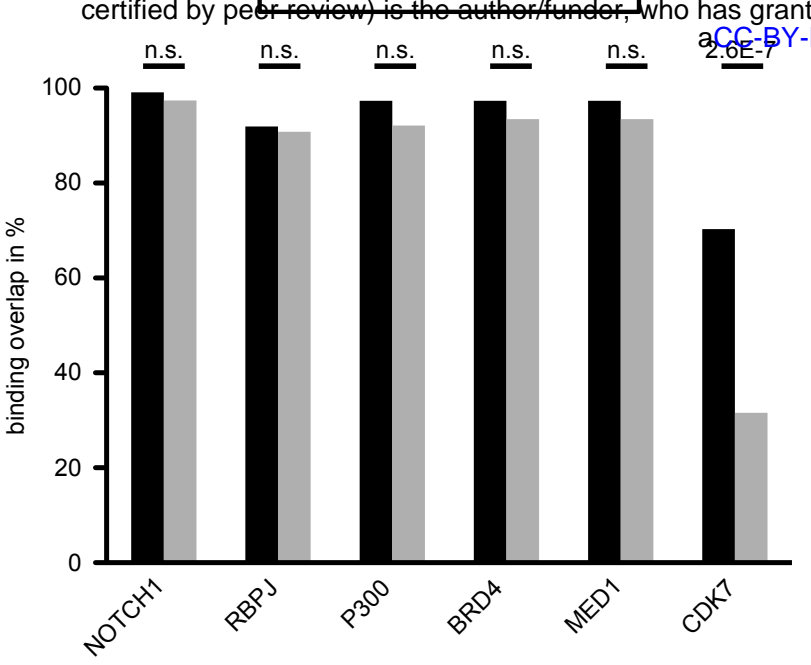

C

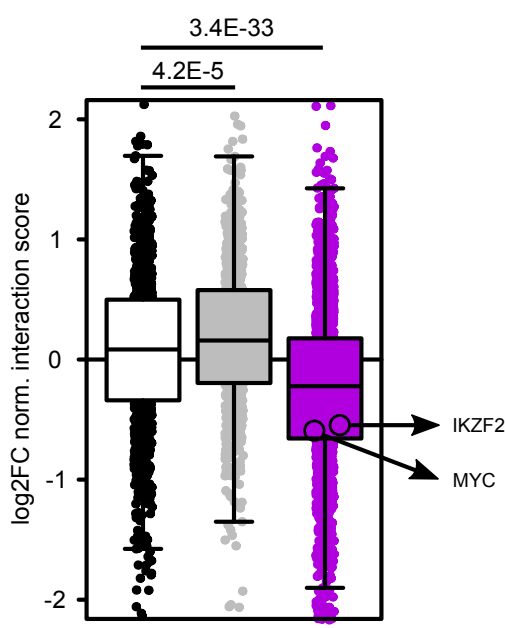

- CUTLL1 DMSO

$\square$ CUTLL1 THZ1

$\square$ THZ1 insensitive

enhancers

$\square$ THZ1 gained

enhancers

$\square$ THZ1 lost

enhancers

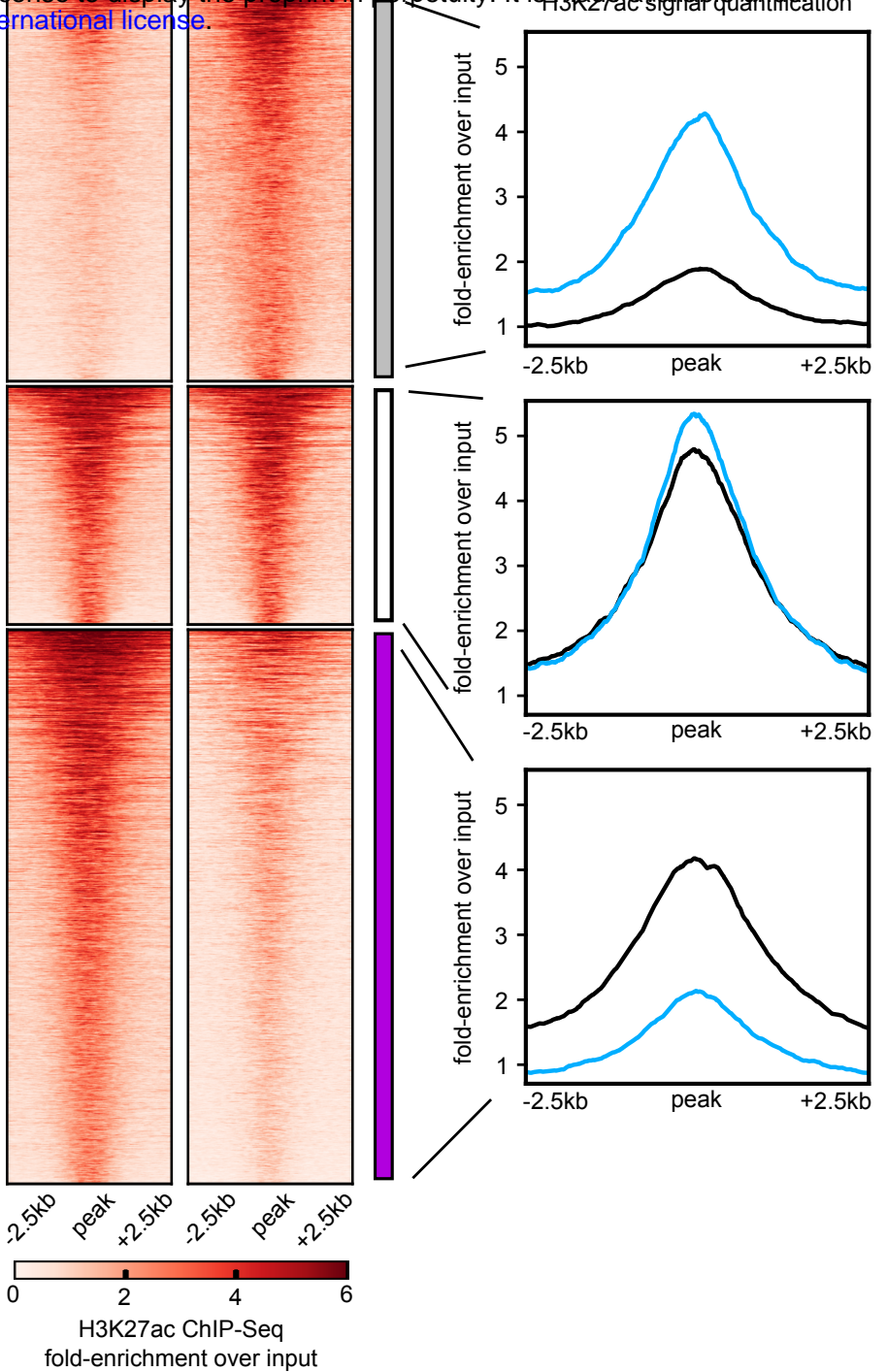

D

CUTLL1 DMSO $\square$ CUTLL1 THZ1

$40 \mathrm{~kb}$ resolution

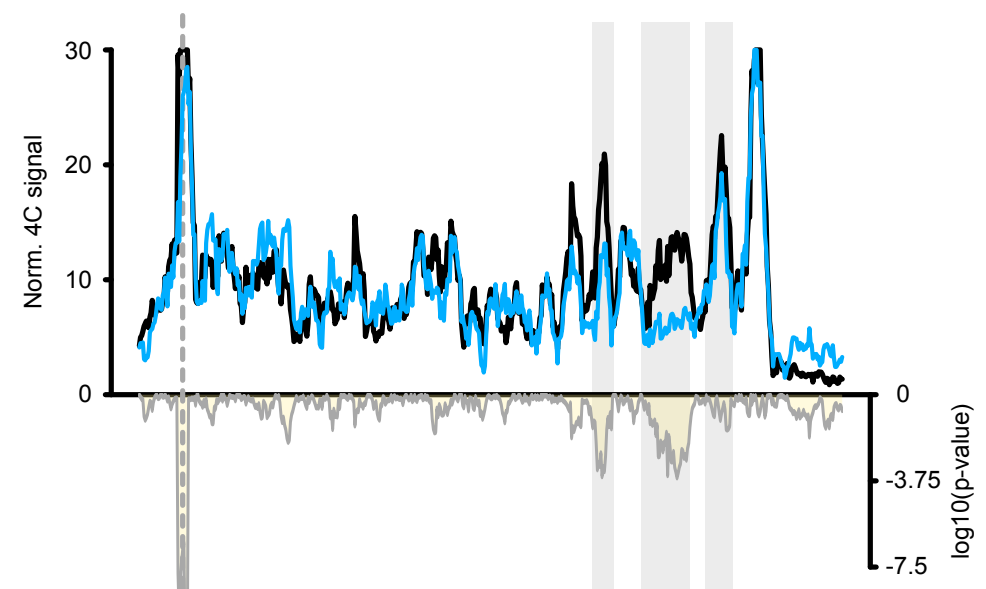

\section{(1)}

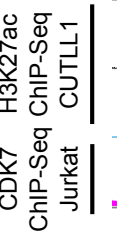

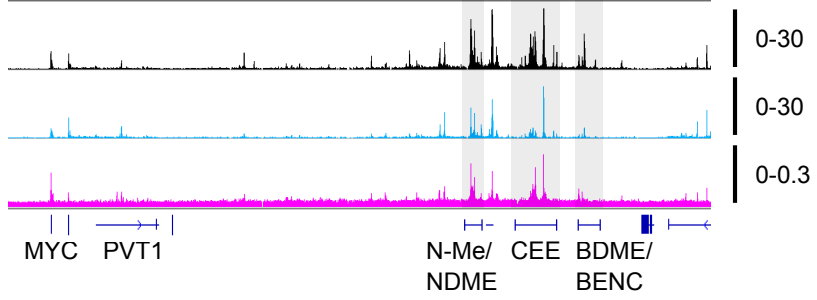

E

chr2:213,875,000-214,200,000

- CUTLL1 DMSO

$\square$ CUTLL1 THZ1 $10 \mathrm{~kb}$ resolution viewpoint
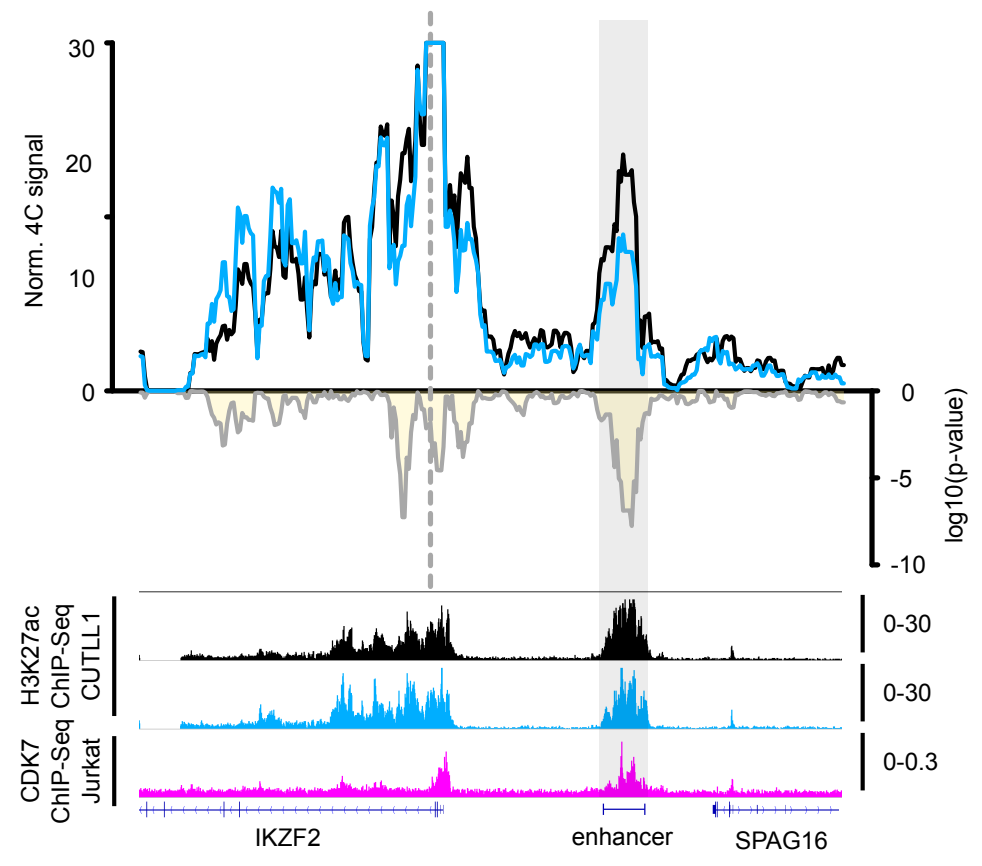


\section{Supplementary Figure 1}

A
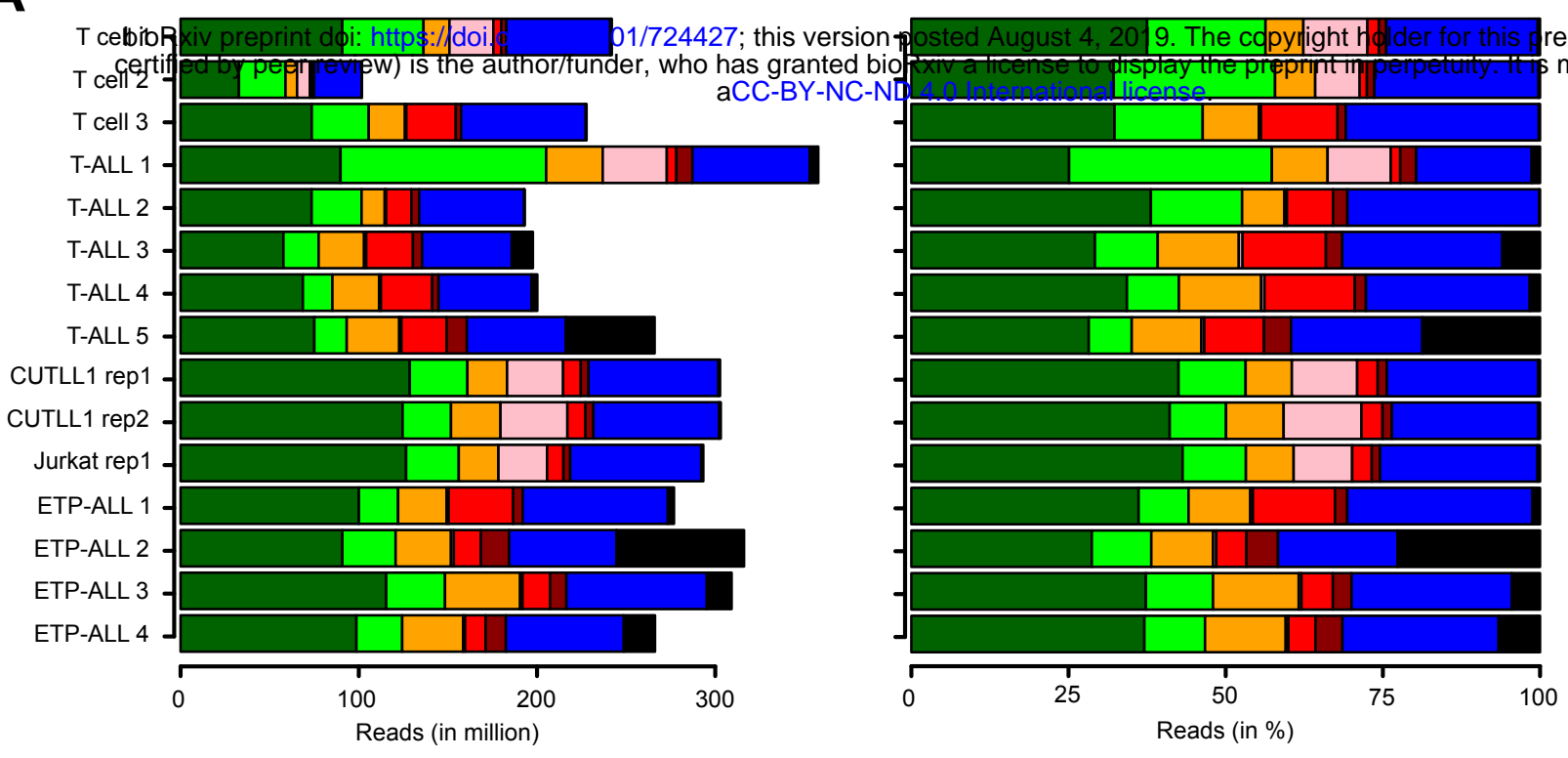

reprint (which was not made available under

B

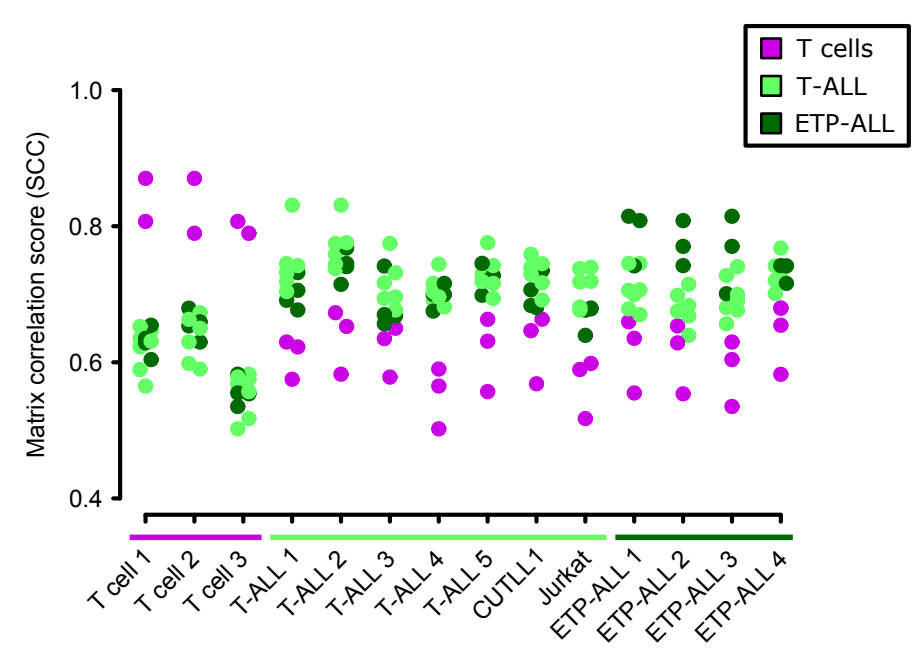

C
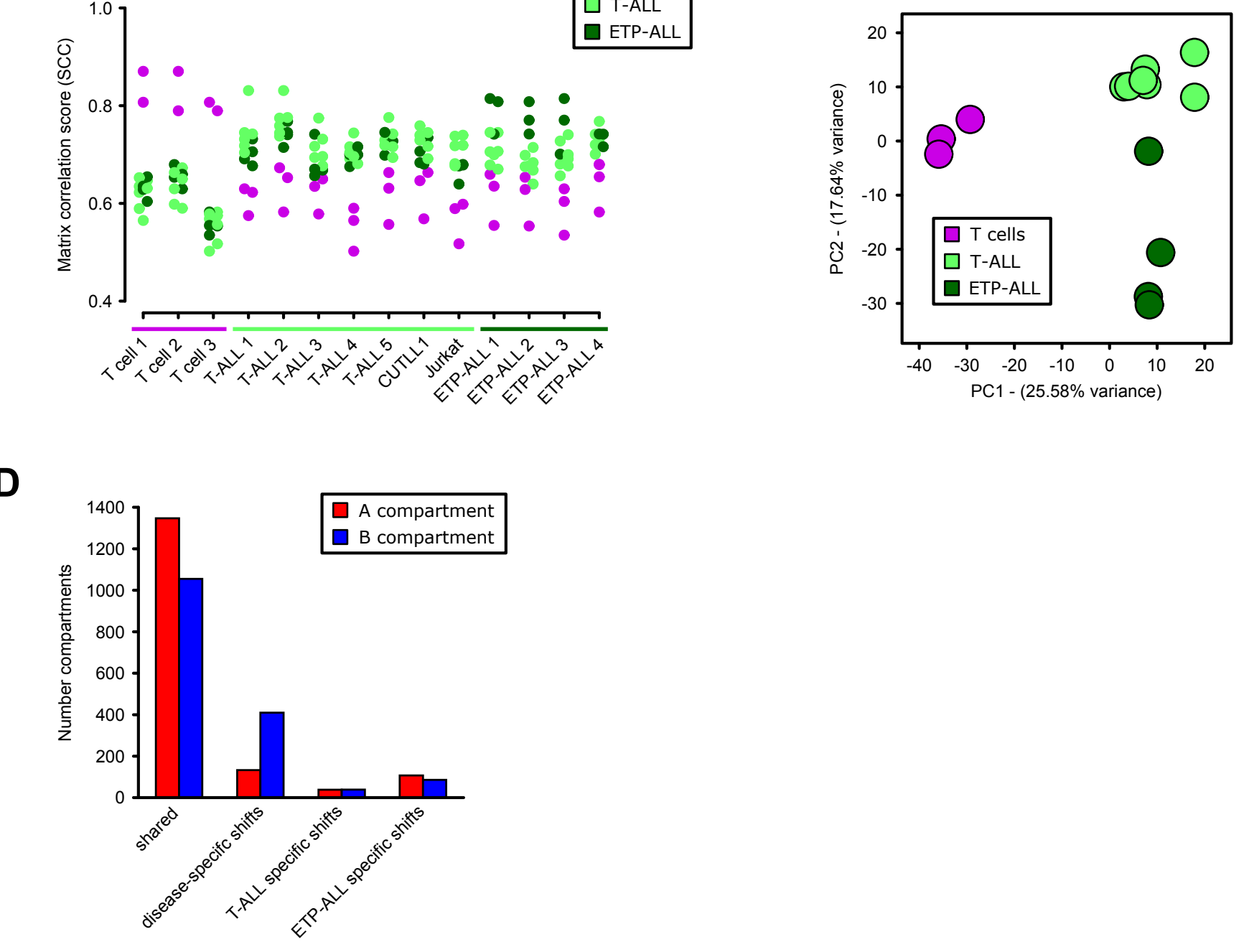


\section{Supplementary Figure 2}

A

B

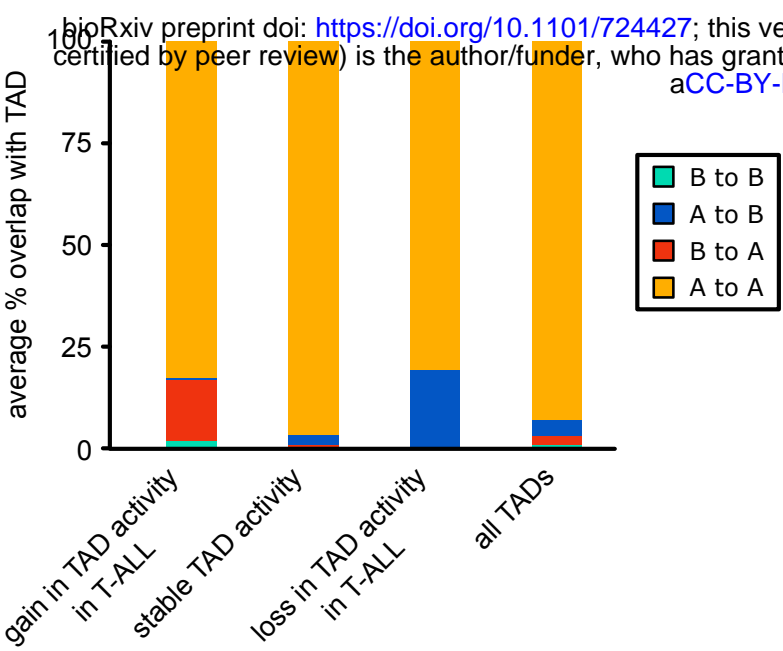

C

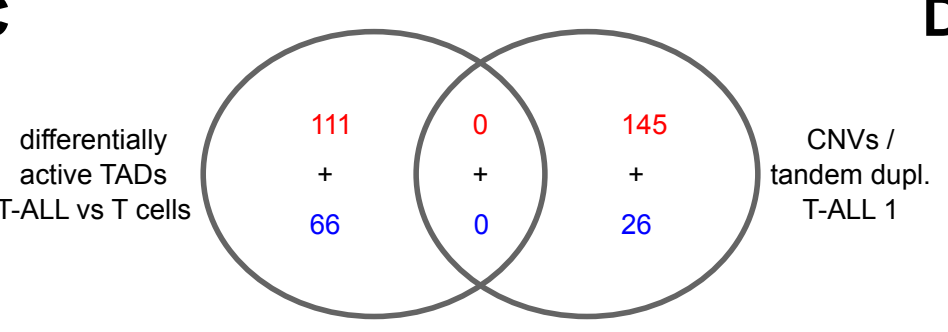

D

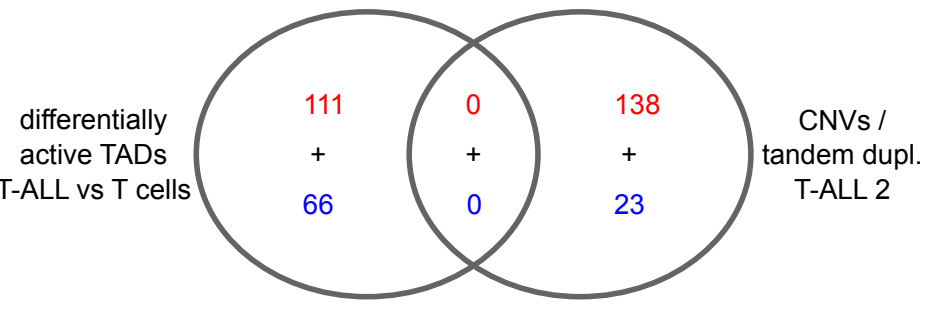

TAD boundaries of differentially active TADs

T-ALL vs T cells

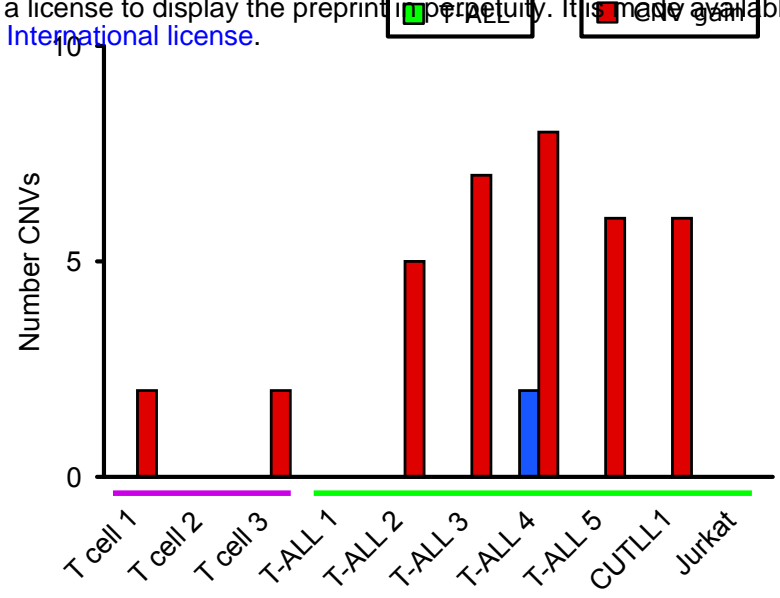

$\mathbf{E}$

T-ALL 1

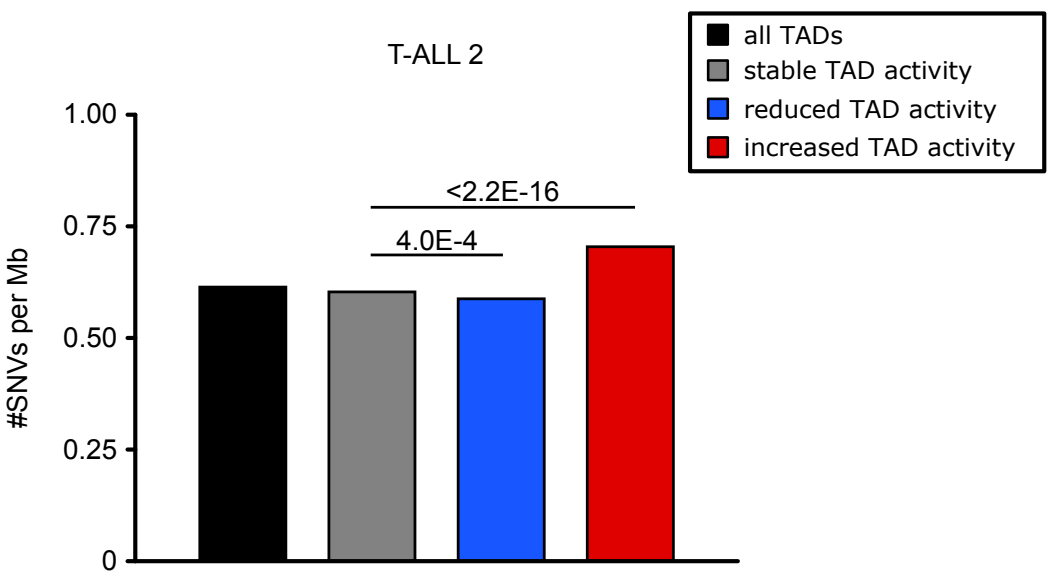

$\mathbf{F}$

SNVs in motifs of differential CTCF binding

$$
\text { ब }
$$

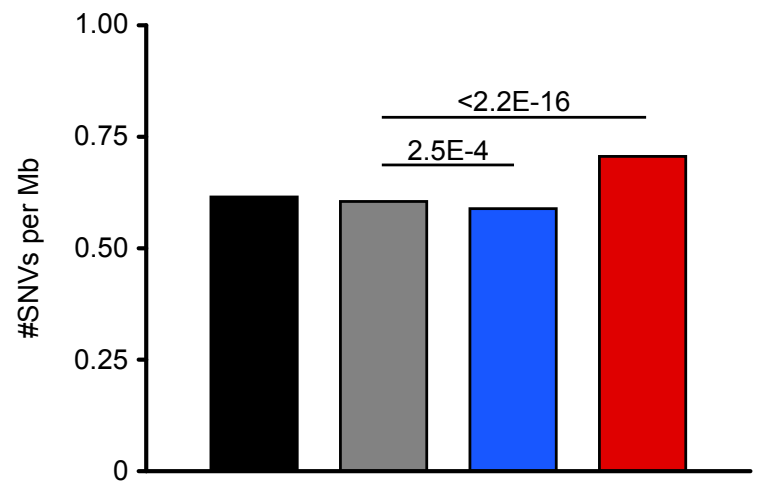

G

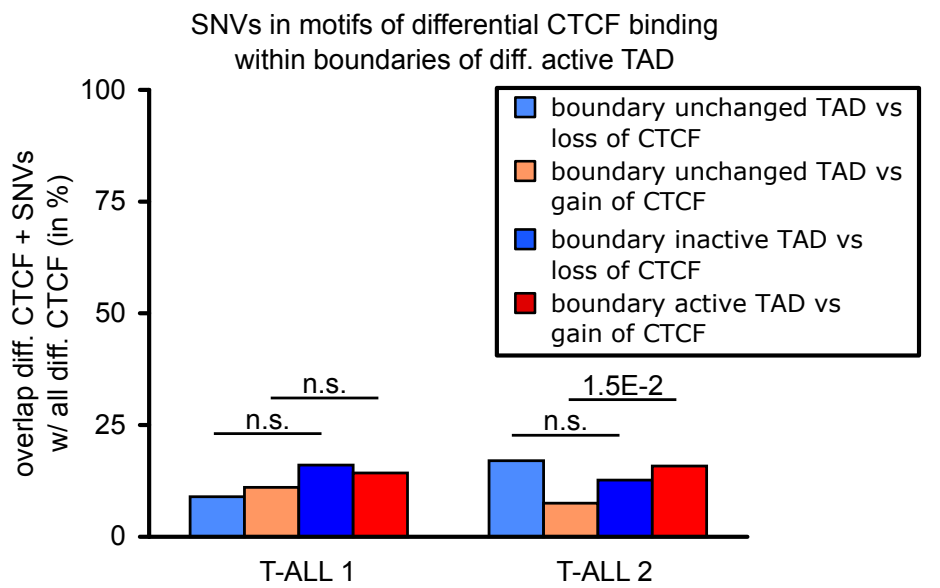




\section{Supplementary Figure 3}

A

chr2:213,300,000-214,900,000

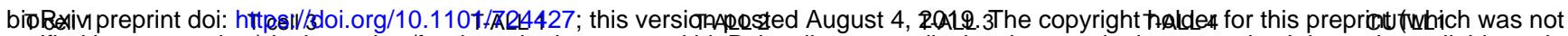
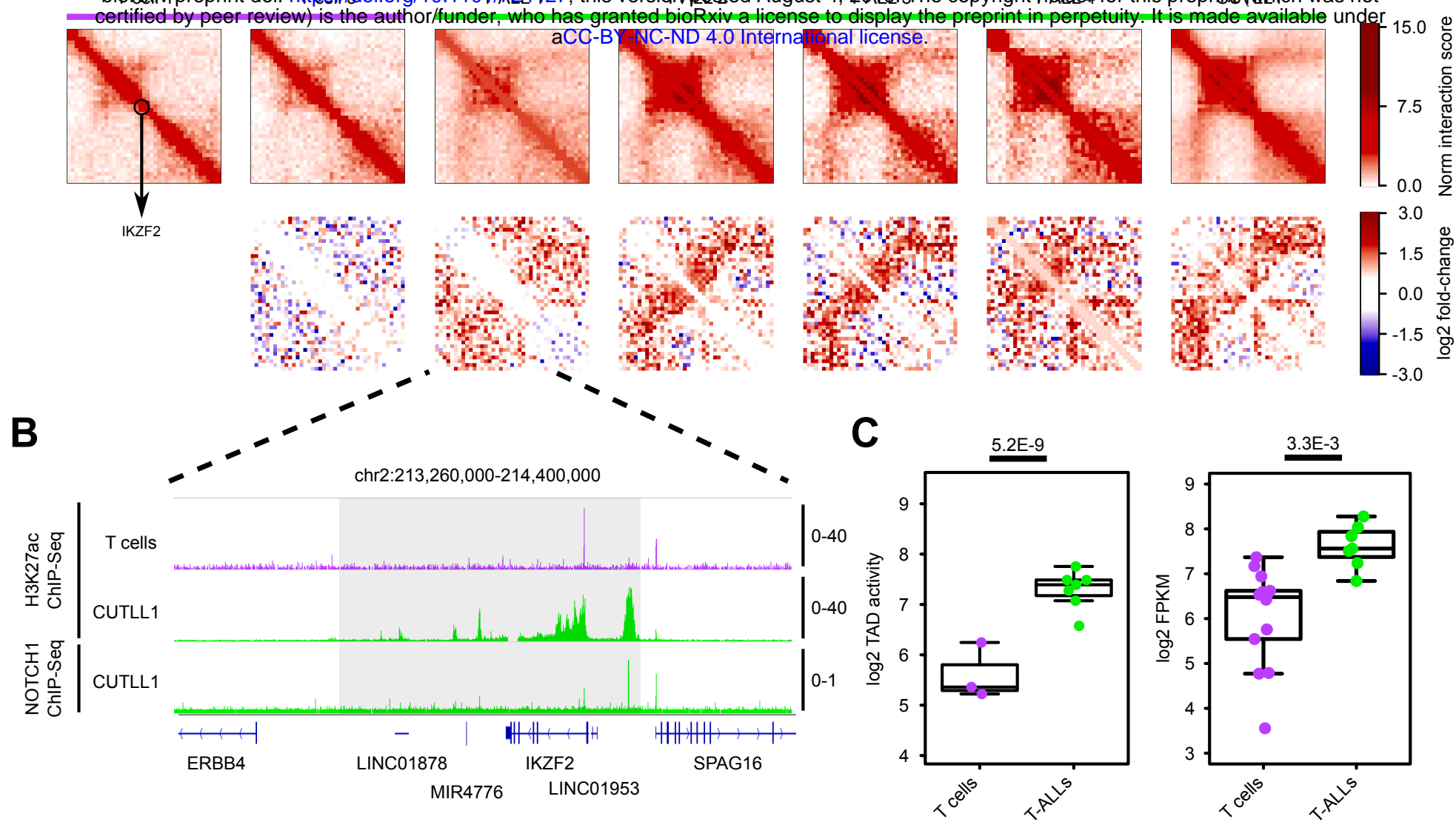

D

T cell 1
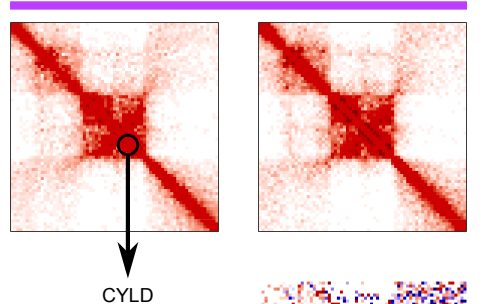

CYLD
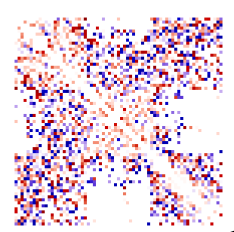

MIR4776

chr16:49,120,000-51,840,000

T-ALL 1
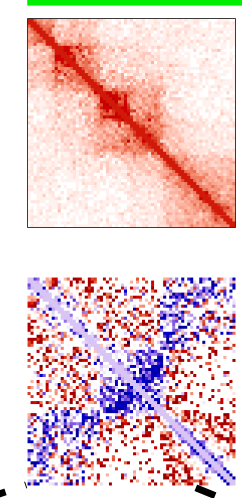

E

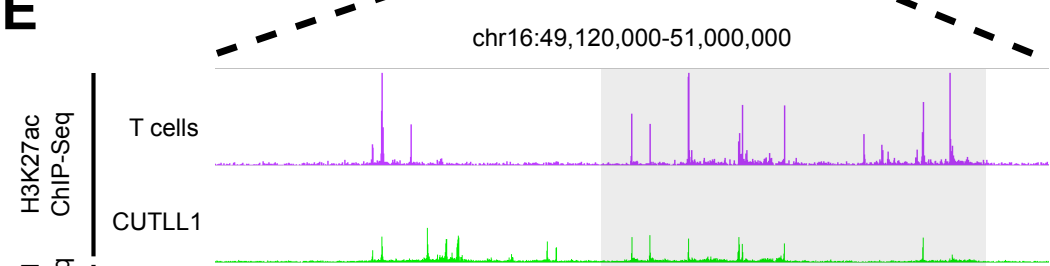

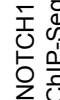

armu

T-ALL 2
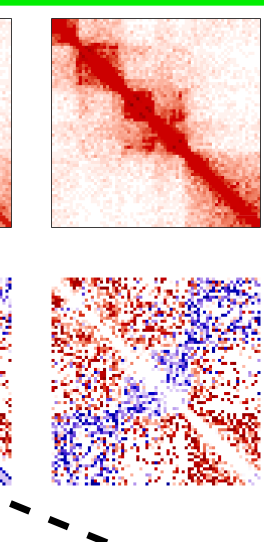

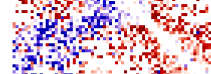

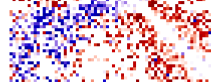
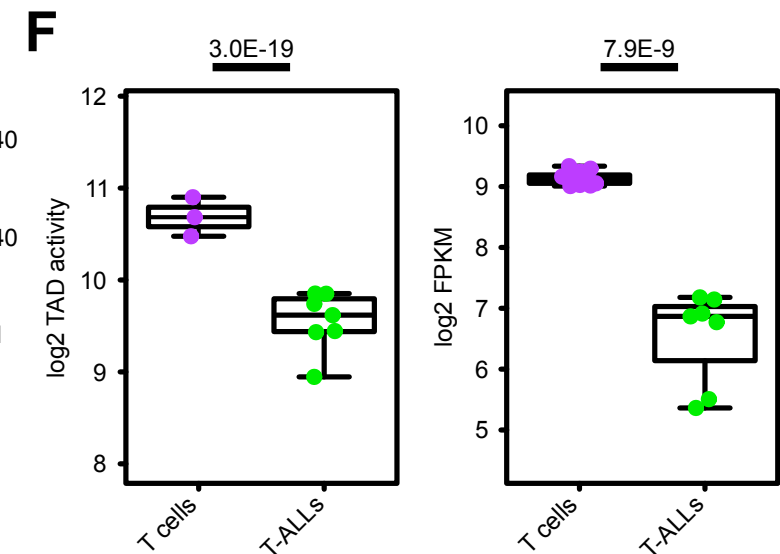


\section{Supplementary Figure 4}

A

B

bioRxiv preprint doi: https://doi.org/10.1101/724427; this version posted August 4, 2019. The copyright holder for this preprint (which was not certified by peer review) is the author/funder, who has granted bioRxiv a license to display the preprint in perpetuity. It is made available under T cells

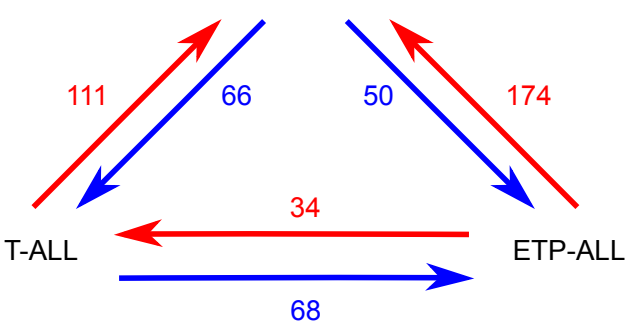
TAAEE-BY-NC-ND 4.0 Internation

C

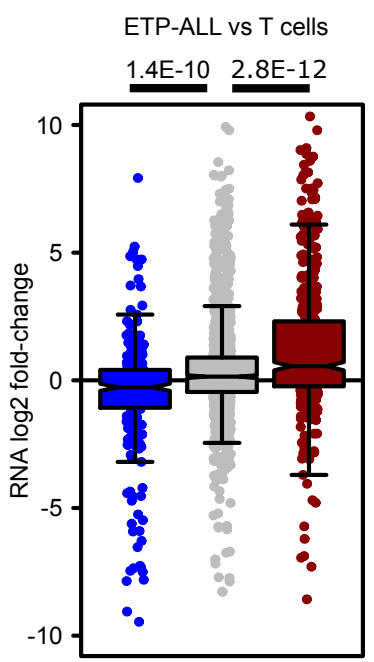

D

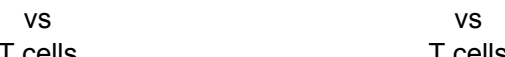

$T$ cells T cells

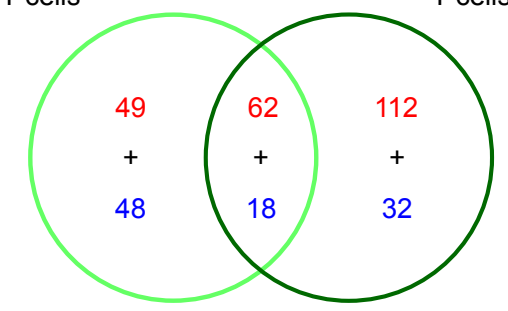

T-ALL vs ETP-ALL 1.6E-10 9.2E-3

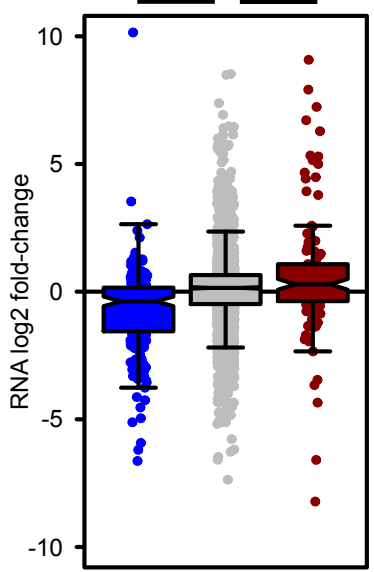

Gain in TAD activity in ETP-ALL

$\square$ Stable TAD activity

$\square$ Loss in TAD activity in ETP-ALL 


\section{Supplementary Figure 5}

A

B

bioRxiv preprint đoi: https://ooiorg/10.1101/724427; this version posted August 4, 2019. The copyright holder for this preprint (Which was not certified by peer review) is the author/funder, who has granted bioRxiv a license to display the preprint in perpeturty. It is made available under

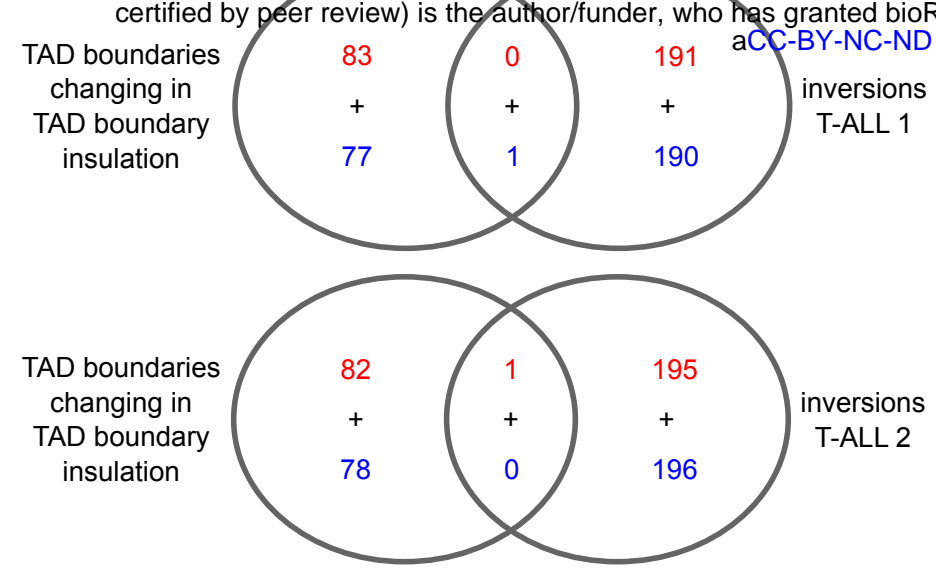

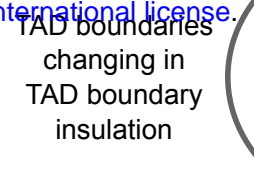

81

81
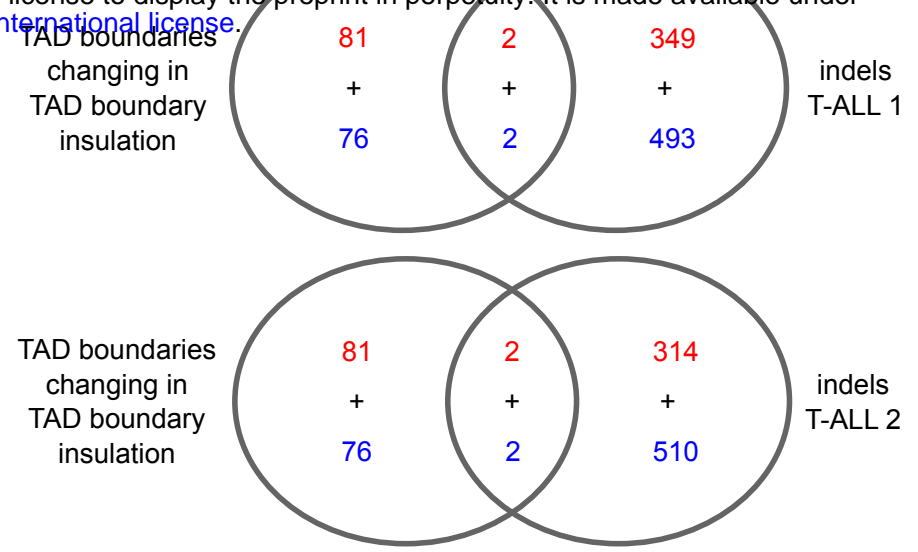


\section{Supplementary Figure 6}

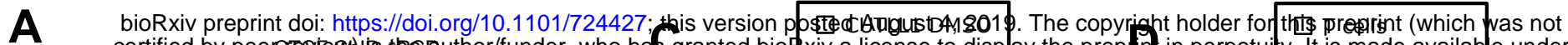

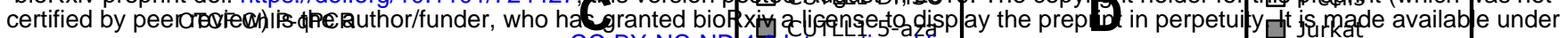

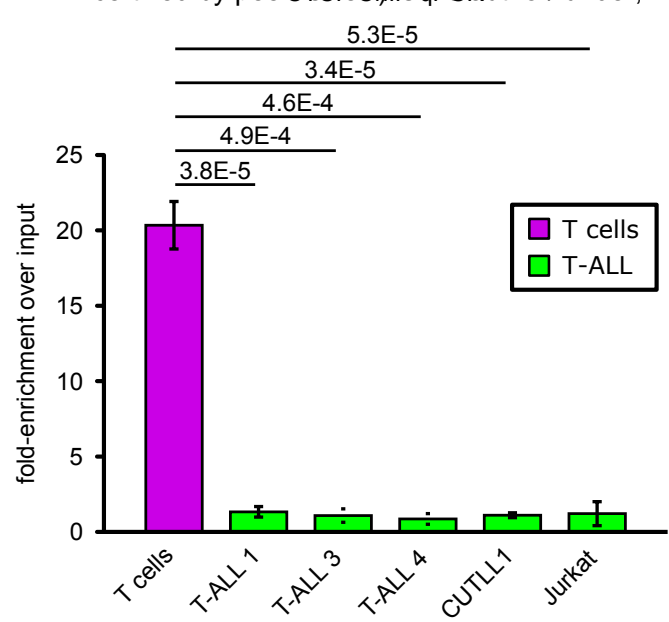
aCC-BY-NC-ND 45 infernationallicense.
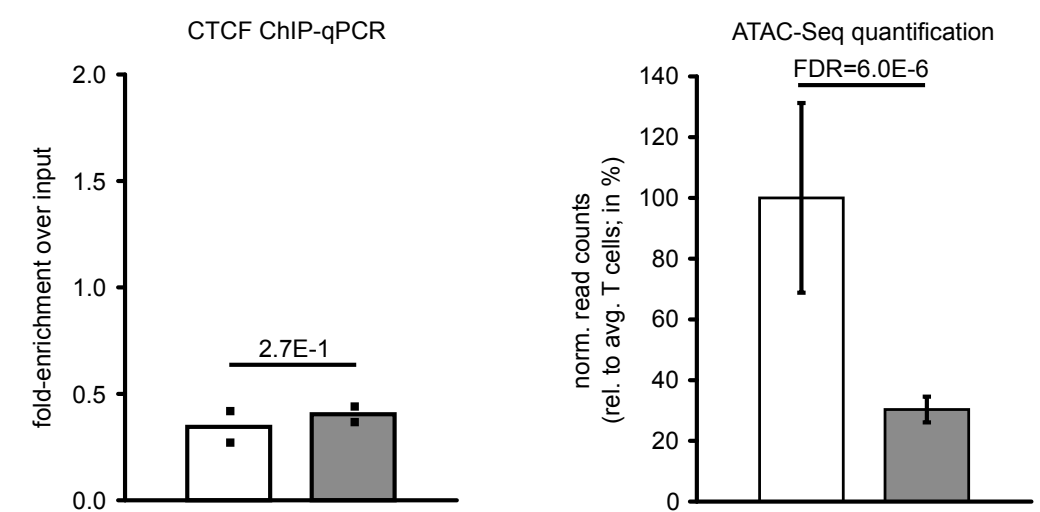

B

Targeted Sanger sequencing

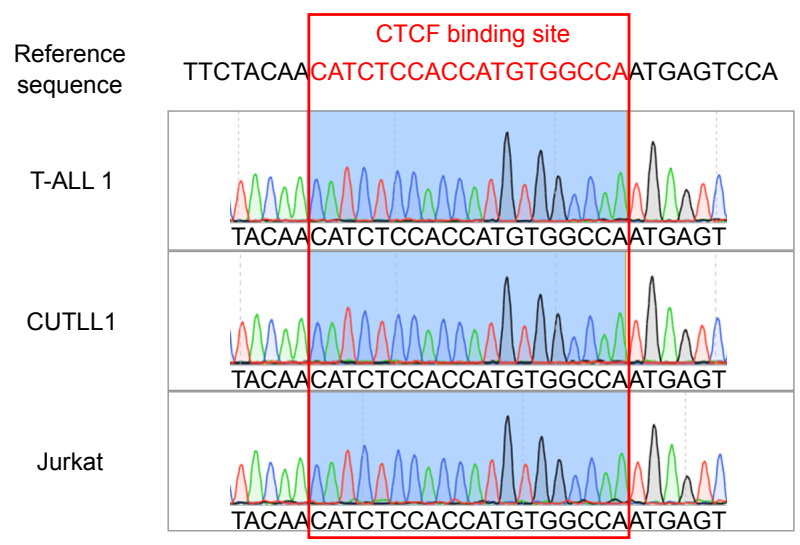

Whole genome sequencing

Reference sequence

T-ALL 1

T-ALL 2 CTCF binding site TTCTACAACATCTCCACCATGTGGCCAATGAGTCCA

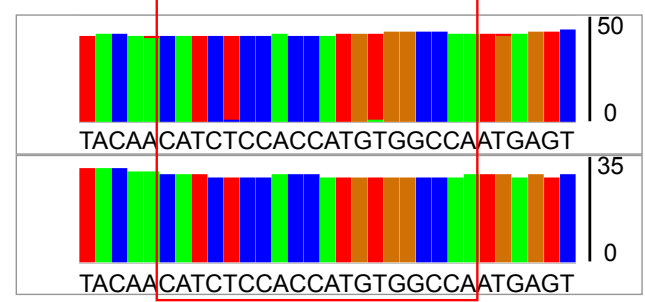




\section{Supplementary Figure 7}

A
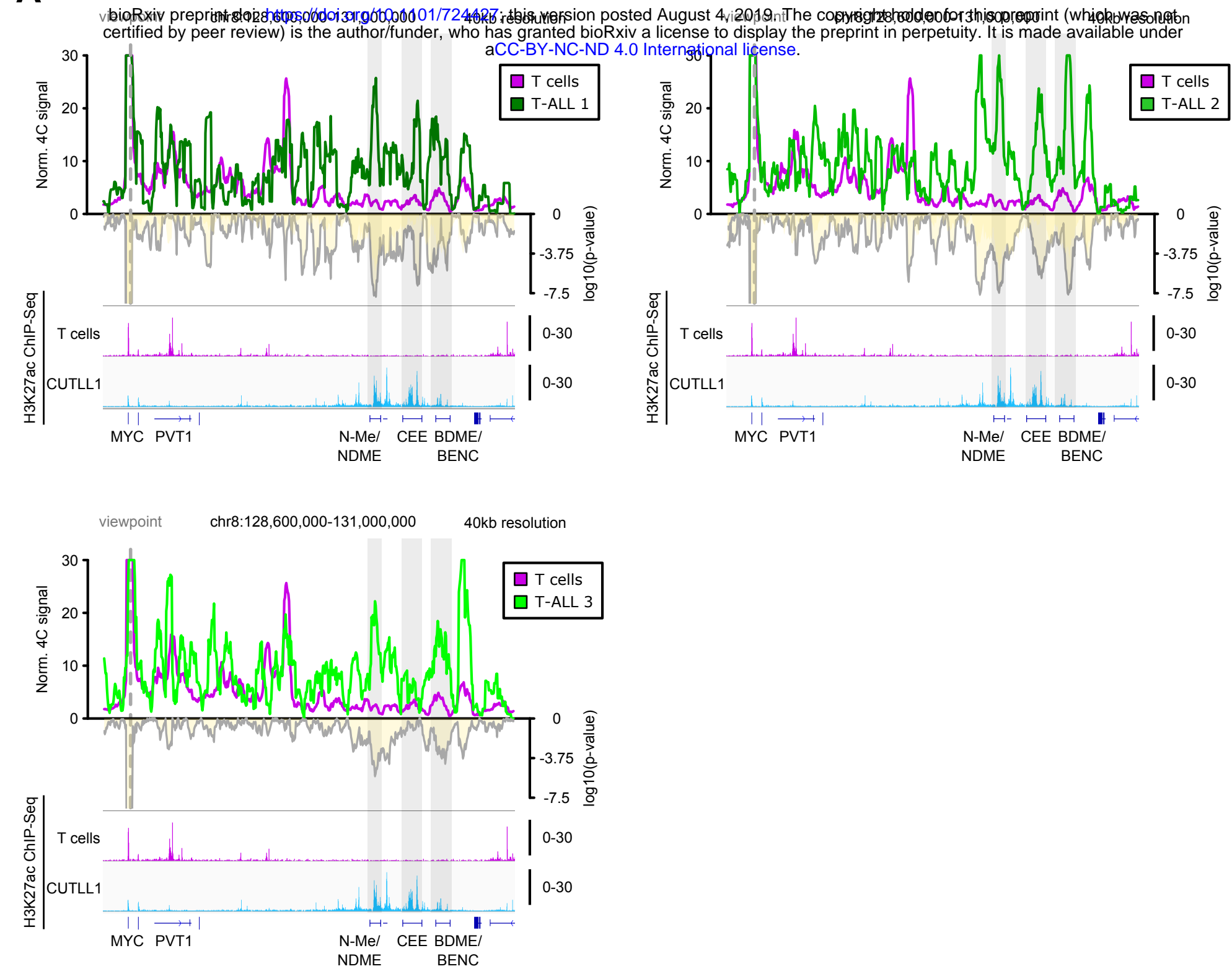


\section{Supplementary Figure 8}

A

bioRxiv preprint doi: https://doi.org/10.1101/724427; this version posted August 4, 2019. The copyright holder for this preprint (which was not

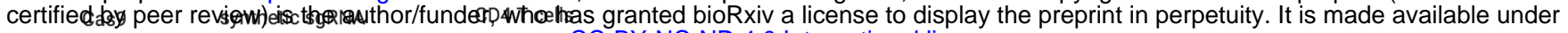

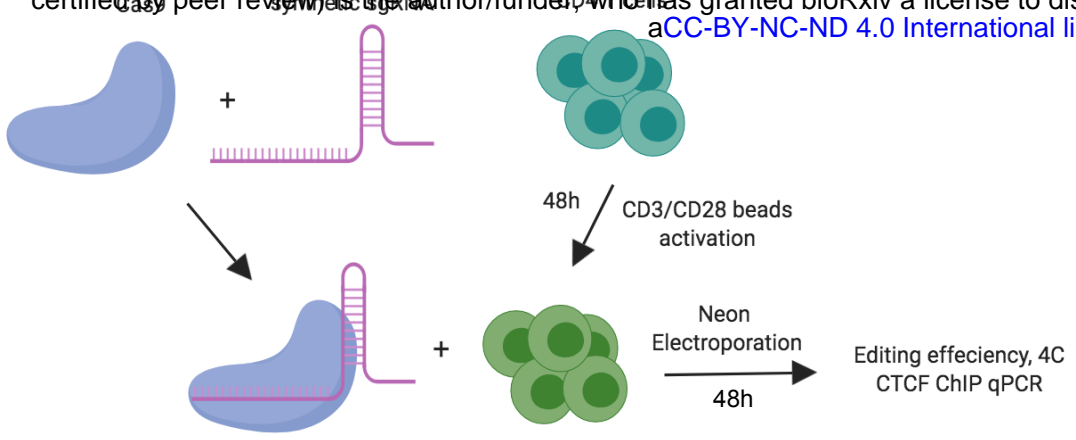

B

INDEL CONTRIBUTION — SEQUENCE

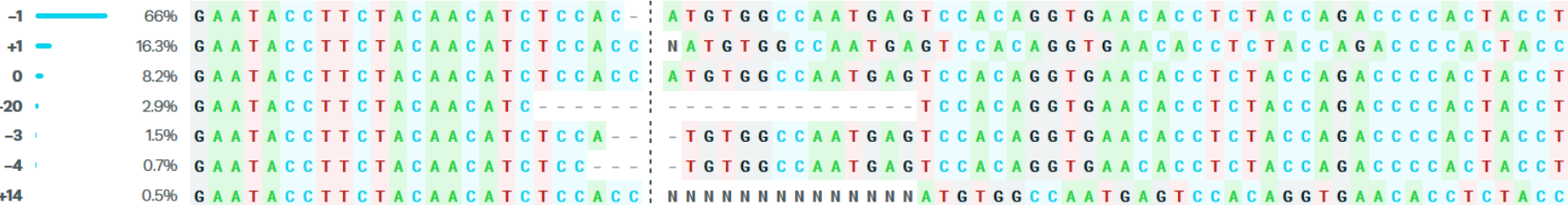

C

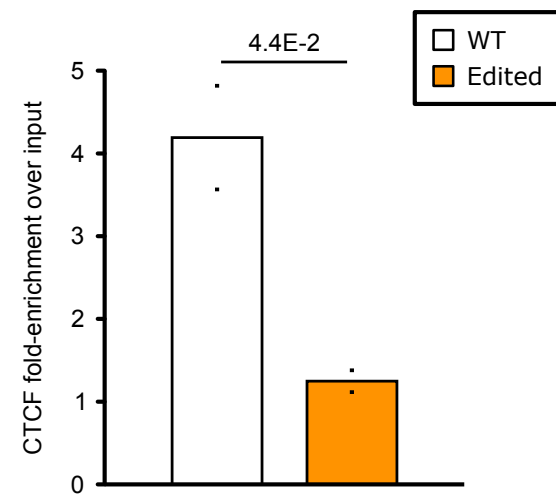

D

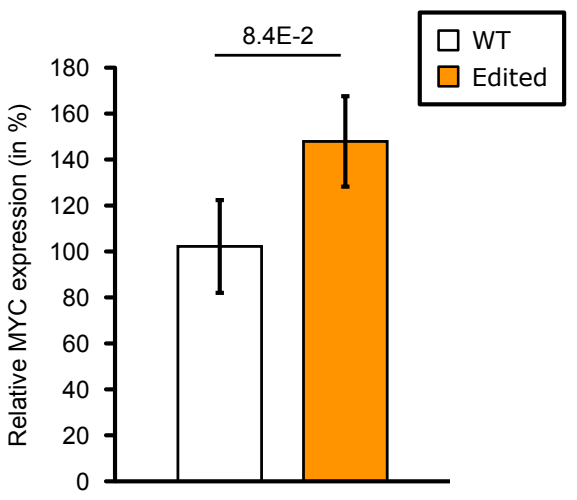

E viewpoint

$\frac{\pi}{2}$

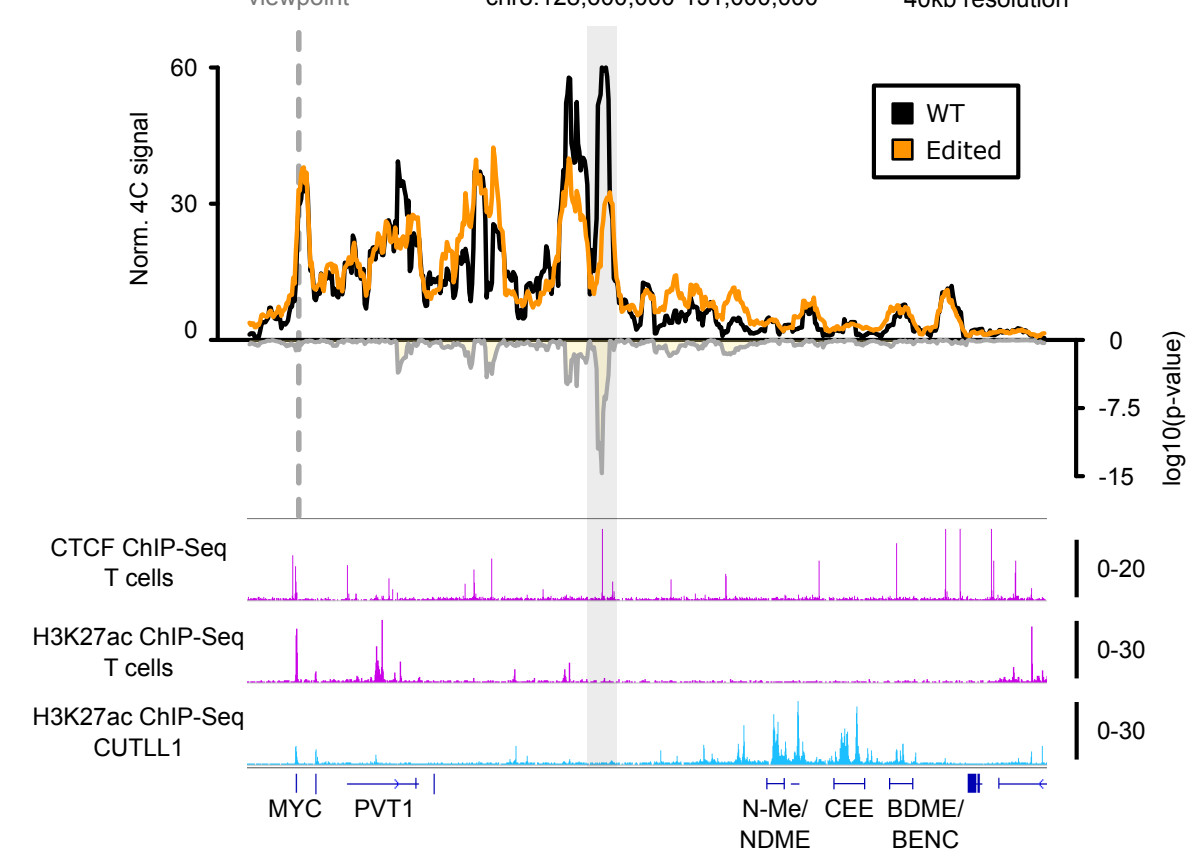

chr8:128,600,000-131,000,000

$40 \mathrm{~kb}$ resolution 


\section{Supplementary Figure 9}

A

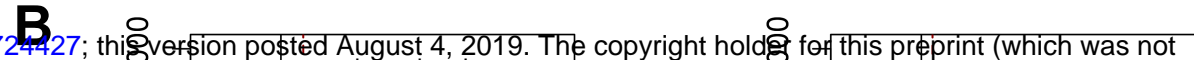

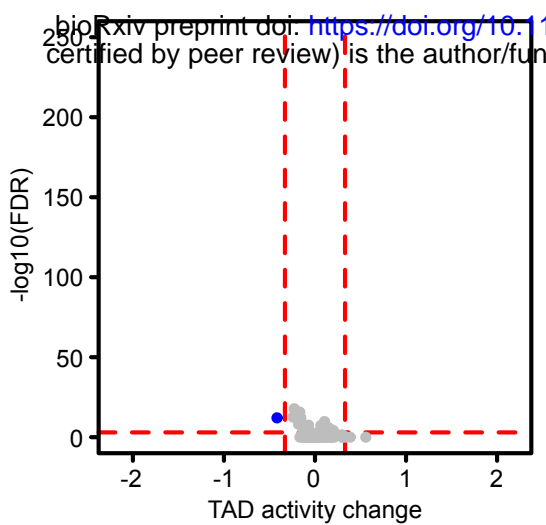
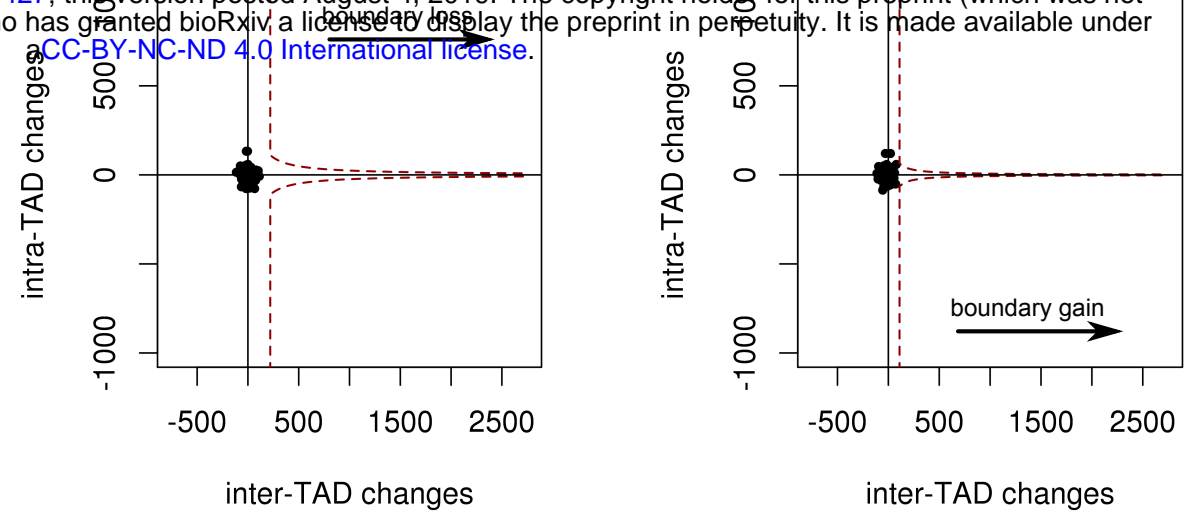

C

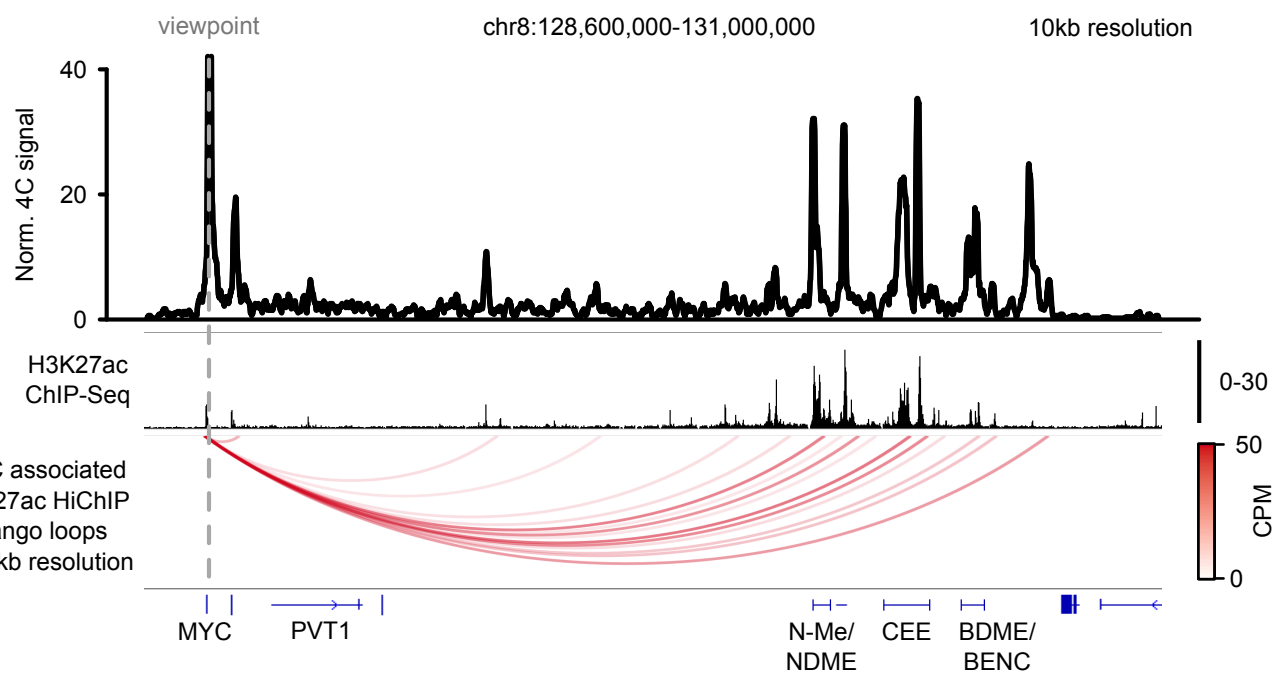

D

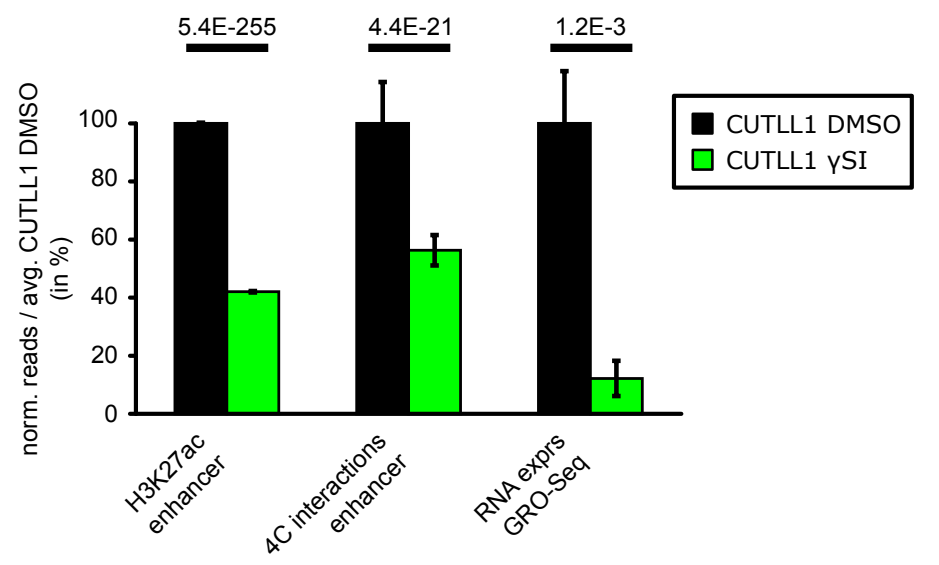

$\mathbf{F}$ NOTCH1 binding (DMSO) NOTCH1 binding (YSI)

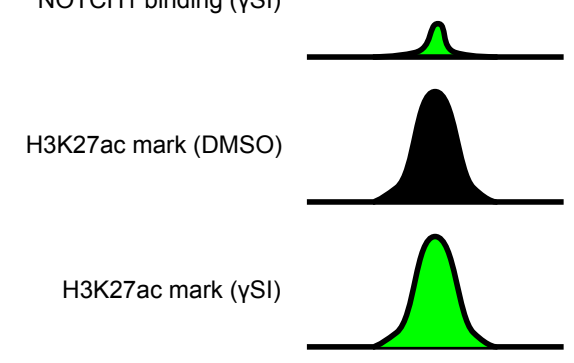

ySI-insensitive enhancer

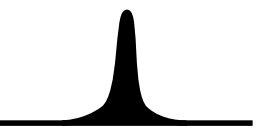

E

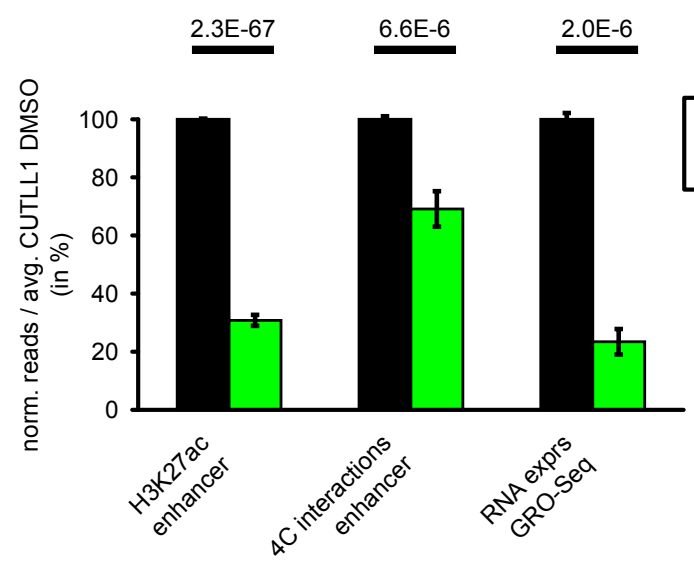

G ySI-sensitive enhancer
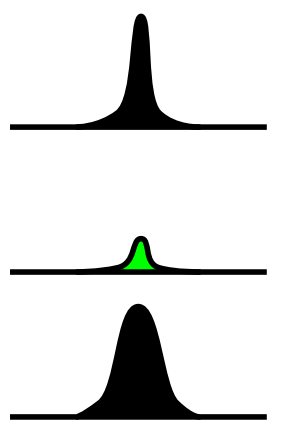
APCDD1 


\section{Supplementary Figure 10}

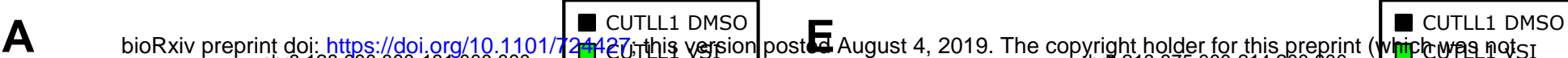

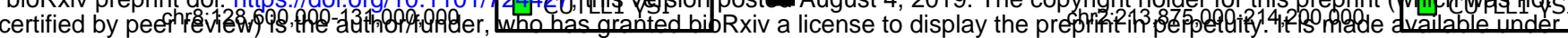

viewpoint $\quad 40 \mathrm{~kb}$ resolution $\mathrm{aCY}-\mathrm{BY}-\mathrm{NC}-\mathrm{ND} 4.0$ International license.

$10 \mathrm{~kb}$ resolution
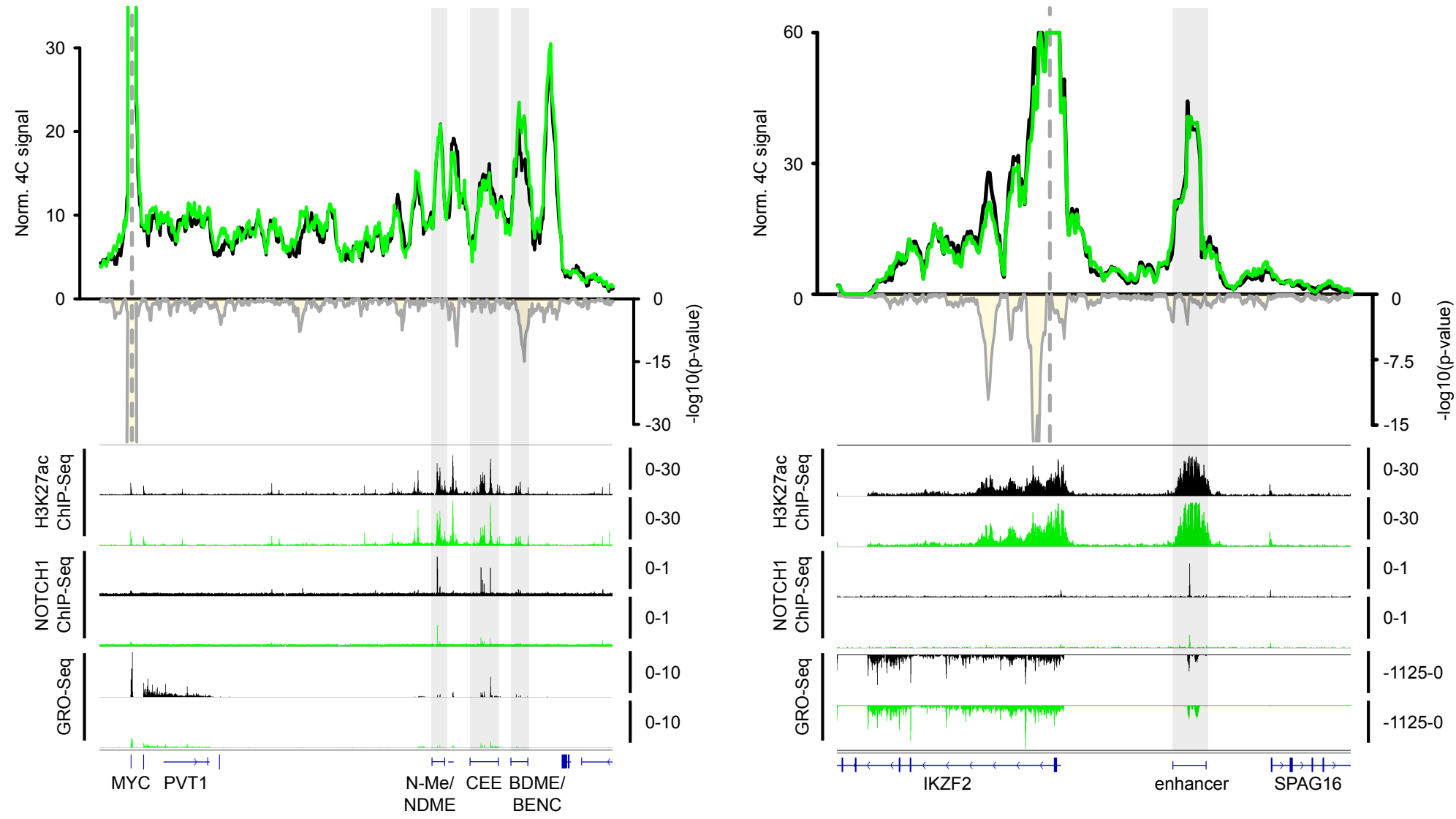

B

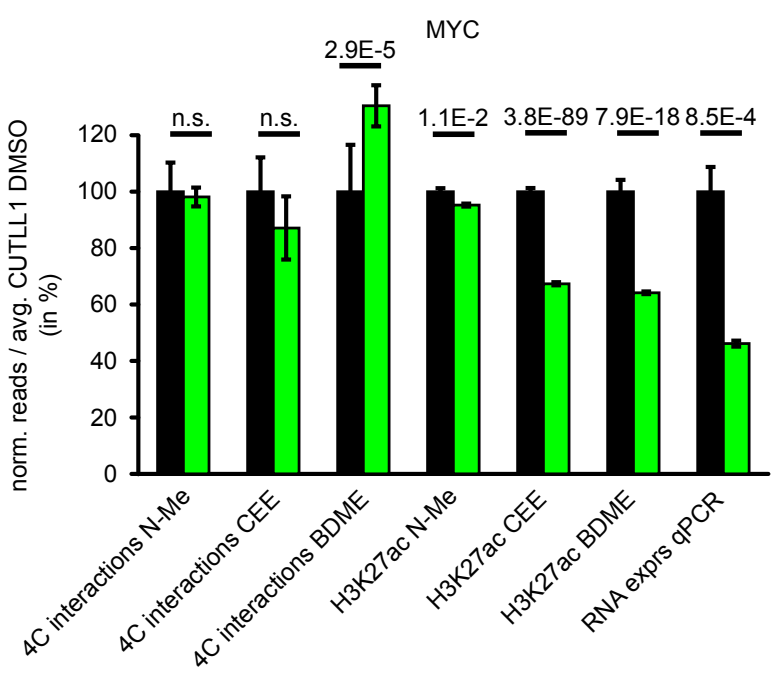

$\mathbf{F}$ IKZF2

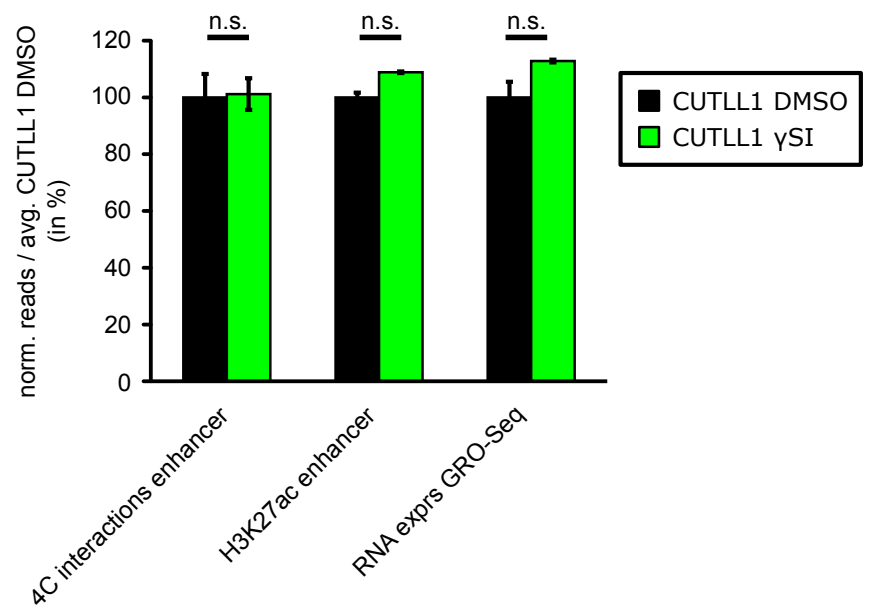

C

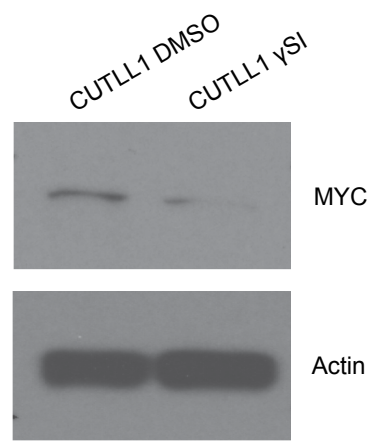

D

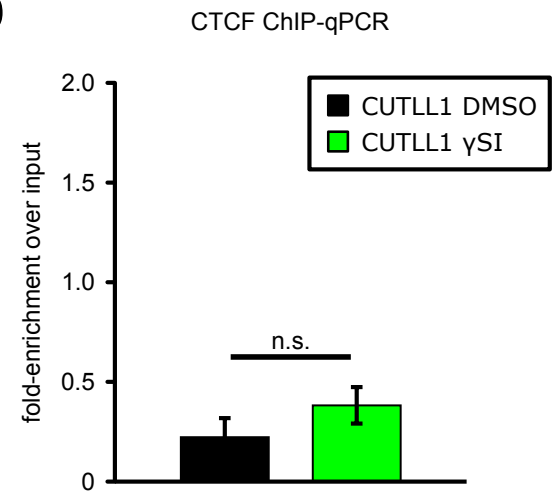




\section{Supplementary Figure 11}

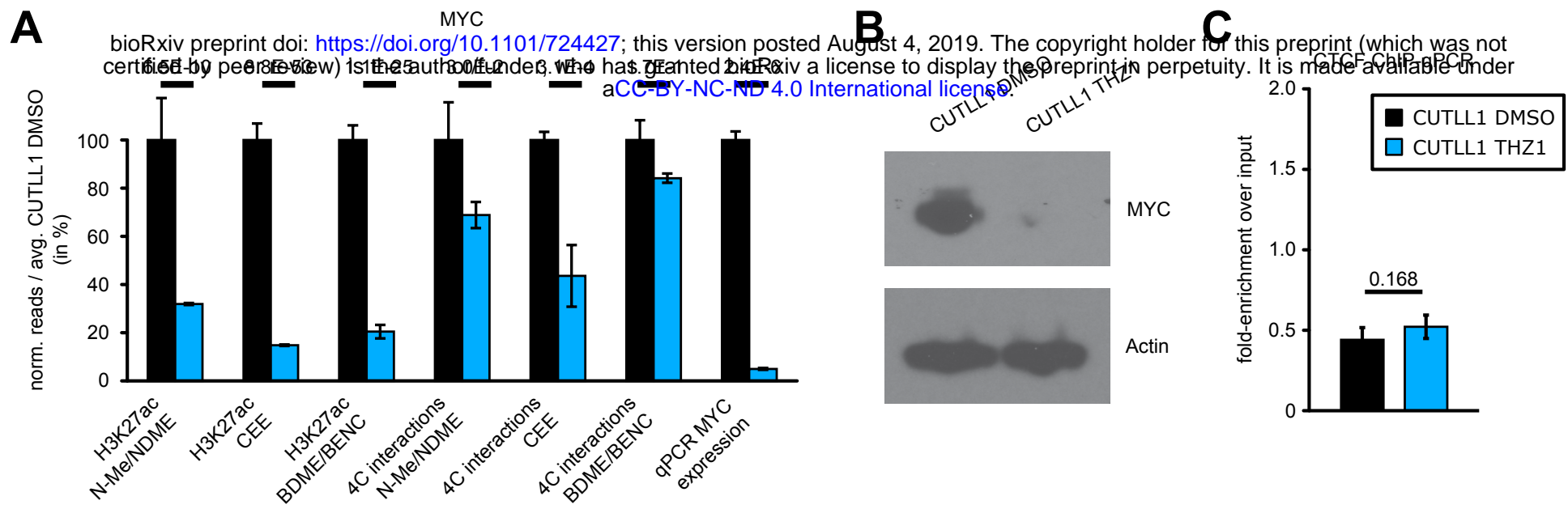

D
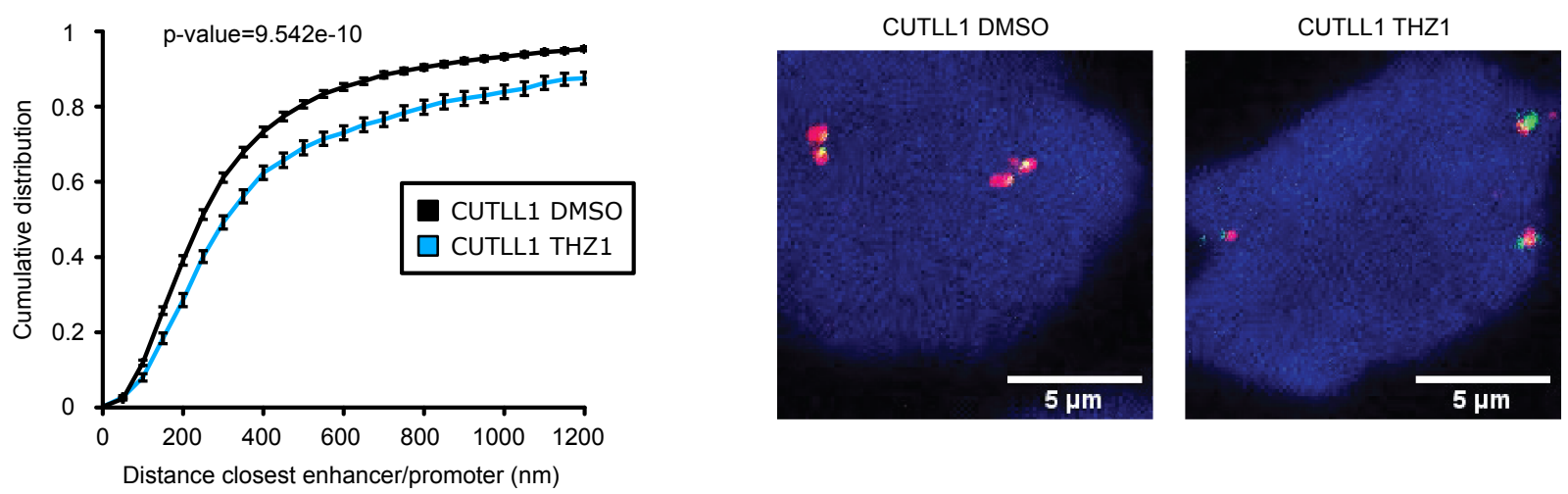

MYC promoter O MYC-CEE $O$ DAPI

E

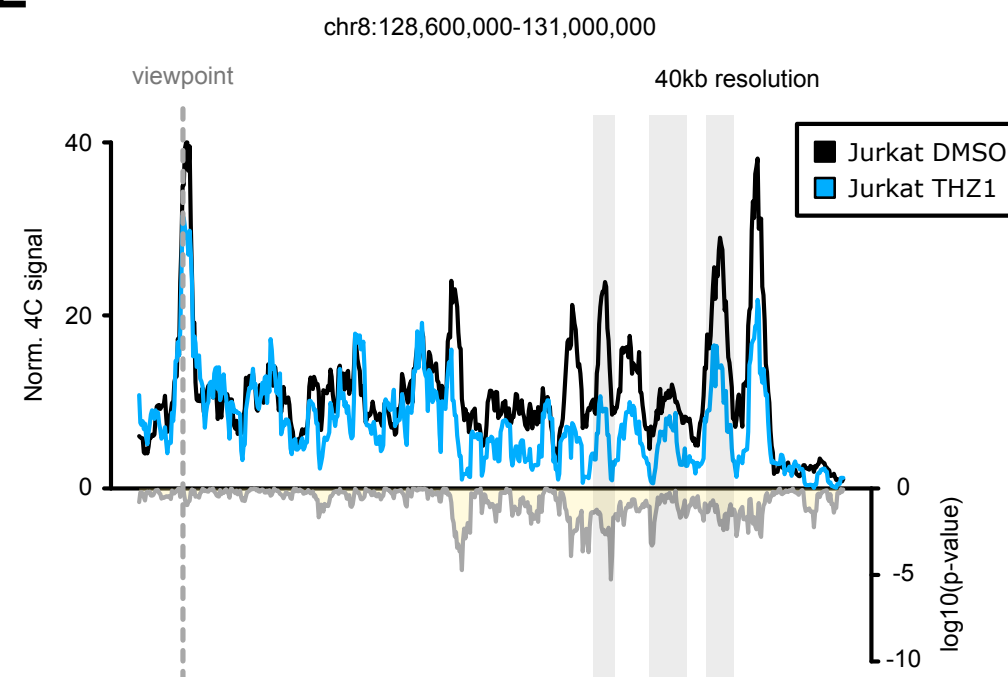

F
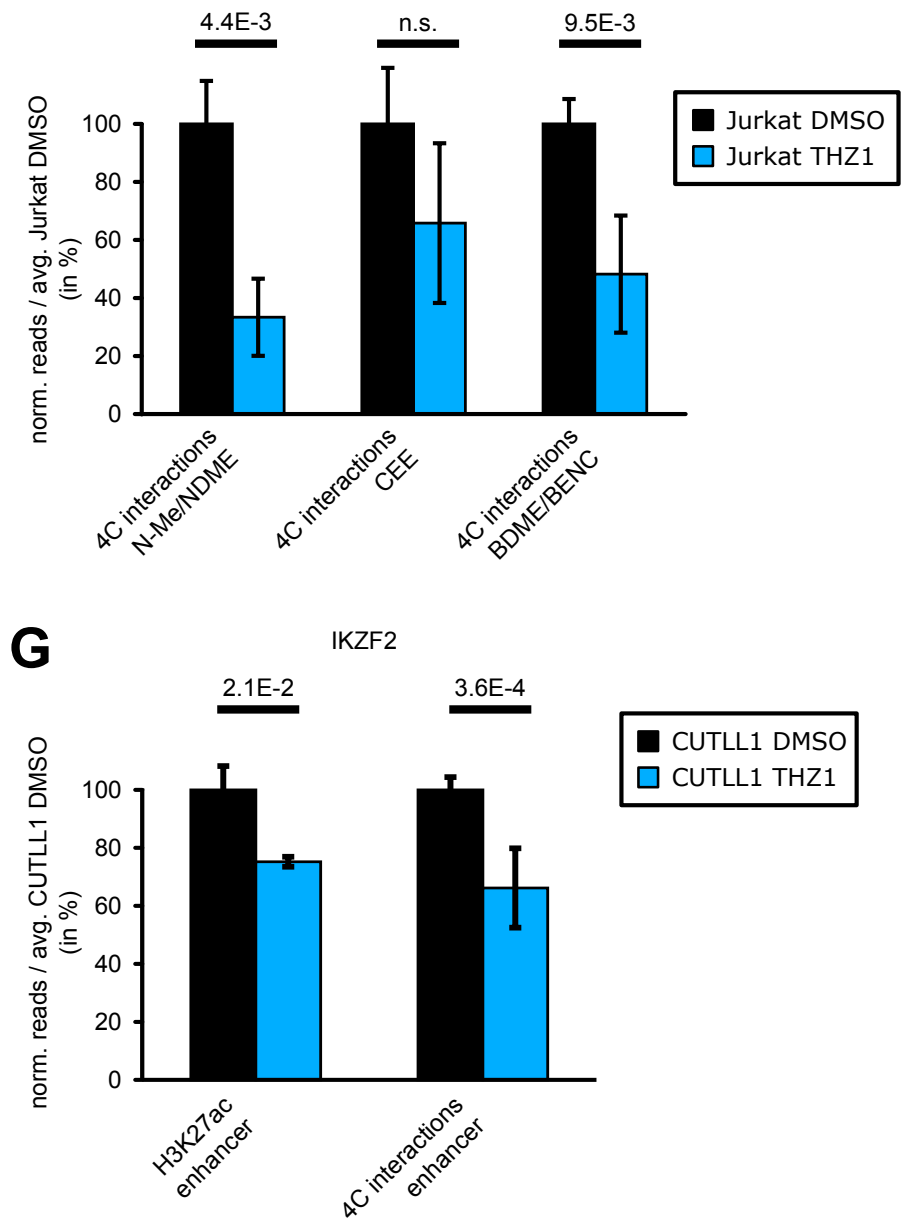

CUTLL1 DMSO $\square$ CUTLL1 THZ1

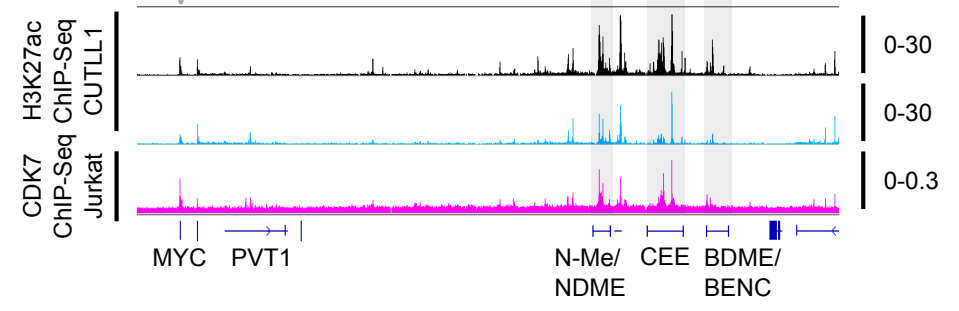




\section{Dynamic 3D chromosomal landscapes in acute leukemia}

\section{Materials and Methods}

\section{Cell culture}

Human cell lines CUTLL1, Jurkat and were cultured in RPMI-1640 media supplemented with $10 \%$ fetal bovine serum, penicillin, streptomycin and glutamine. Tissue culture reagents were purchased from Gibco. Naïve CD4 T cells were purchased from Lonza and cultured in X-vivo 15 culture medium (Lonza) substituted with 5\% human serum (Gemini Bioproducts) and 10 $\mathrm{ng} / \mathrm{ml}$ human IL2 at a density of $10^{6}$ cells per $\mathrm{ml}$.

\section{Primary T-ALL samples}

Primary T-ALL patient samples were collected by Columbia Presbyterian Hospital with informed consent and approved and analyzed under the supervision of the Columbia University Medical Center Institutional Review Board. For expansion of these cells, $1 \times 10^{6}$ patient cells were transplanted into immunodeficient NOD SCID gamma (NSG) mouse strains via retroorbital injection as previously performed ${ }^{1}$. Cells collected from the spleen of these primary recipients were used for the in-situ Hi-C experiment. All the mouse experiments were performed as per ethical guidelines set by IACUC and NYU.

\section{In-situ Hi-C}

Hi-C was performed as described in Rao et al. ${ }^{2}$ Primary samples have been processed as one replicate and all cell line experiments were processed in two biological replicates each. Briefly, 20 million cells were fixed in $1 \%$ formaldehyde for $10 \mathrm{~min}$. Fixed cells were permeabilized in $1 \mathrm{ml}$ lysis buffer $(10 \mathrm{mM}$ Tris- $\mathrm{HCl} \mathrm{pH} 8,10 \mathrm{mM} \mathrm{NaCl}, 0.2 \%$ NP-40, protease inhibitor cocktail (Sigma) for $15 \mathrm{~min}$ on ice, spun down $\left(2000 \times \mathrm{g}, 5 \mathrm{~min}, 4^{\circ} \mathrm{C}\right)$, and the cell pellets were resuspended in $345 \mu 1$ of $1 \times$ NEBuffer2 (NEB) per 5 million cell aliquot. $38 \mu 1$ of $1 \%$ SDS was added to each aliquot, followed by incubation at $65^{\circ} \mathrm{C}$ for $10 \mathrm{~min} .43 \mu \mathrm{l}$ of $10 \%$ Triton $\mathrm{X}-100$ was then added to quench the SDS. To digest chromatin, $400 \mathrm{U}$ of HindIII (NEB) was added per aliquot and incubated at $37^{\circ} \mathrm{C}$ overnight with continuous agitation $(900 \mathrm{rpm})$. After digestion, restriction sites were filled in with Klenow (NEB) in the presence of biotin-14-dATP (Life 
Technologies), dCTP, dGTP and dTTP for 2 hours at $37^{\circ} \mathrm{C}$. Blunt-end ligation was performed by adding 700 $\mu \mathrm{l}$ ligation mix (containing 50U of the T4 DNA ligase (Invitrogen), followed by overnight incubation at $16^{\circ} \mathrm{C}$.

The cross-links were reversed by adding $50 \mu \mathrm{l}$ of $10 \mathrm{mg} / \mathrm{ml}$ proteinase K (Invitrogen) per aliquot and incubated at $65^{\circ} \mathrm{C}$ for 2 hours, followed by addition of another $50 \mu 110 \mathrm{mg} / \mathrm{ml}$ proteinase $\mathrm{K}$ and incubated overnight. All the aliquots per replicate were pooled and DNA was extracted by phenol/chloroform extraction protocol. RNA was digested by adding $1 \mu 1 \mathrm{of} 1 \mathrm{mg} / \mathrm{ml}$ RNase A (Sigma) and incubated at $37^{\circ} \mathrm{C}$ for $30 \mathrm{~min}$. Biotin was removed from non-ligated restriction fragment ends by incubating $40 \mu \mathrm{g}$ of DNA with T4 DNA polymerase (NEB) for 4 hours at $20^{\circ} \mathrm{C}$ in the presence of dATP and dGTP. After DNA purification (Amicon Ultra 30K) and sonication (Covaris E220), the sonicated DNA was double-size selected using Ampure XP beads (Beckman Coulter, $0.8 \mathrm{X}$ - 1.1 X). End-repair was performed using T4 DNA polymerase (NEB), T4 DNA polynucleotide kinase (NEB), Klenow (NEB) and dNTPs in 1× T4 DNA ligase reaction buffer (NEB), followed by dATP-addition with Klenow. Biotin-marked ligation products were isolated with MyOne Streptavidin C1 Dynabeads (Life Technologies). Paired-end (PE) adapters (Illumina) were ligated to DNA fragments using $15 \mathrm{U}$ of the T4 DNA ligase (Invitrogen) for 2 hours at room temperature. Bead-bound DNA was amplified with 6 PCR amplification cycles using PE PCR 1.0 and PE PCR 2.0 primers (Illumina). Primary samples T-ALL 2-5, T cell donor 2 and ETP-ALL samples along with CUTLL1 DMSO and THZ1 treated samples were processed with the commercial Arima genomics HiC Kit (https://arimagenomics.com/) and processed according to manufactures guidelines. The concentration and size distribution of Hi-C library DNA after PCR amplification was determined by tapestation (Agilent Technologies), and the HiC libraries were sequenced on Illumina Hi-Seq 2500 or Illumina Hi-seq 4000 at 50 cycles.

\section{ChIP-Seq}

ChIP-seq was performed as described previously ${ }^{3}$. All H3K27ac ChIP-Seq experiments were performed in biological duplicates. CTCF ChIP-Seq experiments for primary samples were performed as biological duplicates. For cell line experiments, we performed five replicates for CUTLL1, three replicates for CUTLL1 $\gamma$ SI experiments and two replicates for CUTLL1 JQ1. For all conditions we created a single input sample. In brief, 5 million cells were fixed in $1 \%$ formaldehyde and snap frozen in liquid nitrogen and stored in $-80{ }^{\circ} \mathrm{C}$ till usage. For Histone 
chips, 2 million cells were crosslinked as previously described. Nuclei were isolated from the fixed cells using the nuclei isolation buffer $(15 \mathrm{mM}$ Tris $\mathrm{pH} 7.5,60 \mathrm{mM} \mathrm{KCl}, 15 \mathrm{mM} \mathrm{NaCl}, 15 \mathrm{mM}$ $\mathrm{MgCl} 2,1 \mathrm{mMCaCl} 2,250 \mathrm{mM}$ Sucrose, $1 \mathrm{mM}$ DTT and Protease inhibitor). The isolated nuclei were lysed using nuclei lysis buffer (50 mM Tris-HCl (pH 8.0), 10 mM EDTA (pH 8.0) and 1\% SDS). This was followed by sonication (30 mins in total) using the bioruptor from Diagenode at high output with 30s ON and 30s OFF cycles. Following sonication to the desired fragment size of 400-600 bp, the sonicated lysate was diluted with nine volumes of IP dilution buffer $(0.01 \%$ SDS, 1.1\% Triton X-100, 1.2 mM EDTA (pH 8.0), $16.7 \mathrm{mM}$ Tris-HCl pH 8.0 and $167 \mathrm{mM}$ $\mathrm{NaCl}$ ) and magnetic DynaI beads for $1 \mathrm{~h}$ (preclearing of chromatin). Following preclearing, CTCF was immunoprecipitated with $10 \mu \mathrm{l}$ of monoclonal rabbit CTCF antibody, clone D31H2 (Cell Signaling 3418) overnight at $4^{\circ} \mathrm{C}$ or H3K27ac (Active motif; Catalog no: 39133). The purified ChiP DNA was used to generate sequencing libraries using Hapa Hyper prep kit from Kapa Biosystems. Libraries were sequenced in single-end using Illumina Hiseq 2500 or Illumina Hi-seq 4000 at 50 cycles.

\section{RNA-Seq}

RNA-seq libraries were prepared using NEXTflex Rapid Illumina Directional RNA-seq Library prep kit as per manufacturer's guidelines. The libraries were sequenced in single-end by either HiSeq 2500 or HiSeq 4000 at 50 cycles.

\section{C-Seq}

For LUNAR1 and APCDD1 viewpoints, we have created biological duplicates for all experiments. For MYC viewpoint, we have created five biological replicates for CUTLL1 DMSO, three replicates for CUTLL1 $\gamma$ SI and two replicates for CUTLL1 JQ1 and two replicates for T cells. Edited T cells 2 replicates (5 million each); CUTLL1 DMSO and THZ1 treatment, biological triplicates.

For each replicate, 10 million cells were fixed in 2\% formaldehyde and 10\% FBS in PBS for 10 min at room temperature. For edited and WT T cells, 5 million cells were used. Crosslinking was quenched with glycine and the $4 \mathrm{C}$-Seq was performed as described previously ${ }^{4}$. Cells were lysed on ice with $1 \mathrm{ml}$ lysis buffer (50mM Tris $\mathrm{pH} 7.3,150 \mathrm{mM} \mathrm{NaCl}, 5 \mathrm{mM}$ EDTA, 0.5\% NP-40, $1 \%$ Triton $\mathrm{X}-100$ ) for $15 \mathrm{~min}$. Nuclei were spun down and resuspended in $360 \mu \mathrm{l} \mathrm{H} 2 \mathrm{O}$ (or 
frozen). $60 \mu \mathrm{l}$ of 10X DpnII restriction buffer was added along with $15 \mu \mathrm{l} 10 \%$ SDS, and left shaking for $1 \mathrm{hr}$ at $37^{\circ} \mathrm{C}$ followed by addition of $150 \mu \mathrm{l}$ of $10 \%$ Triton X-100 and an additional shaking for $1 \mathrm{~h}$ at $37^{\circ} \mathrm{C}$. $5 \mu \mathrm{l}$ of undigested control was stored, and nuclei were incubated overnight with 200U of DpnII (NEB, R0543M) restriction enzyme. A fresh 200U of DpnII was added the following morning for 6hrs. Following this, the digestion was checked for completion by running $5 \mu \mathrm{l}$ of sample in a $1 \%$ agarose gel. DpnII was inactivated with $80 \mu 110 \%$ SDS, and a proximity ligation reaction was performed in a $7 \mathrm{ml}$ volume using 4000U T4 DNA Ligase (NEB M0202M). Crosslinks were reversed at $65^{\circ} \mathrm{C}$ overnight after adding $300 \mu \mathrm{g}$ Proteinase $\mathrm{K}$. Samples were then treated with $300 \mu \mathrm{g}$ RNAse A for $45 \mathrm{~min}$ at $37^{\circ} \mathrm{C}$, and DNA was ethanol precipitated. A 2 nd restriction digest was performed overnight in a $500 \mu$ l reaction with $50 \mathrm{U}$ Csp61 (Fermentas, ER0211). The enzyme was inactivated at $65^{\circ} \mathrm{C}$ for $25 \mathrm{~min}$, and a proximity ligation reaction was performed in a 14ml volume with 6000 U T4 DNA Ligase. Sample DNA was ethanol precipitated, and purified using the QIAquick PCR purification kit (Qiagen). To generate 4C-Seq library, $1 \mu \mathrm{g}$ of prepared 4C template was amplified 30 PCR cycles per bait per condition (See Supplementary Table for viewpoint sequences) and the amplified fragments were sequenced in Illumina HiSeq 2500 to generate single end reads at 50 cycles.

\section{HiChIP}

HiChIP was performed as previously described ${ }^{5}$ with some modifications. In brief, up to 10 million crosslinked cells were resuspended in $500 \mu \mathrm{L}$ of ice-cold HiC lysis buffer $(10 \mathrm{mM}$ Tris$\mathrm{HCl} \mathrm{pH} \mathrm{7.5,} 10 \mathrm{mM} \mathrm{NaCl}, 0.2 \% \mathrm{NP}-40,1 \times$ protease inhibitors) and rotated at $4^{\circ} \mathrm{C}$ for $30 \mathrm{~min}$. Nuclei were pelleted and washed once with $500 \mu \mathrm{L}$ of ice-cold HiC lysis buffer. Pellet was then resuspended in $100 \mu \mathrm{L}$ of $0.5 \%$ SDS and incubated at $62^{\circ} \mathrm{C}$ for $10 \mathrm{~min} .285 \mu \mathrm{L}$ of water and 50 $\mu \mathrm{L}$ of $10 \%$ Triton $\mathrm{X}-100$ were added, and samples were rotated at $37^{\circ} \mathrm{C}$ for $15 \mathrm{~min}$. $50 \mu \mathrm{L}$ of NEB Buffer 2 and $15 \mu \mathrm{L}$ of $25 \mathrm{U} / \mu \mathrm{L}$ MboI restriction enzyme (NEB, R0147) were then added, and sample was rotated at $37^{\circ} \mathrm{C}$ for $2 \mathrm{~h}$. MboI was then heat inactivated at $62^{\circ} \mathrm{C}$ for $20 \mathrm{~min}$. We added $52 \mu \mathrm{L}$ of incorporation master mix: $37.5 \mu \mathrm{L}$ of $0.4 \mathrm{mM}$ biotin-dATP (Jena Biosciences, NU-835-BIO14-S); $1.5 \mu \mathrm{L}$ of a dCTP, dGTP, and dTTP mix at $10 \mathrm{mM}$ each; and $10 \mu \mathrm{L}$ of 5 U/ $\mu$ L DNA Polymerase I, Large (Klenow) Fragment (NEB, M0210). The reactions were then rotated at $37^{\circ} \mathrm{C}$ for 1 h. $948 \mu \mathrm{L}$ of ligation master mix was then added: $150 \mu \mathrm{L}$ of $10 \times \mathrm{NEB}$ T4 DNA ligase buffer with 10 mM ATP (NEB, B0202), $125 \mu \mathrm{L}$ of $10 \%$ Triton X-100, $3 \mu \mathrm{L}$ of 50 
mg/mL BSA (Thermo Fisher, AM2616), $10 \mu \mathrm{L}$ of $400 \mathrm{U} / \mu \mathrm{L}$ T4 DNA Ligase (NEB, M0202), and $660 \mu \mathrm{L}$ of water. The reactions were then rotated at room temperature for $4 \mathrm{~h}$. After proximity ligation, the nuclei were pelleted and the supernatant was removed. The nuclear pellet was brought up to $880 \mu \mathrm{L}$ in Nuclear Lysis Buffer $(50 \mathrm{mM}$ Tris-HCl pH 7.5, $10 \mathrm{mM}$ EDTA, 0.5\% SDS, 1× Roche protease inhibitors, 11697498001), and sonicated with a Bioruptor 300 (Diagenode) for 8 cycles of $30 \mathrm{sec}$ each, on a medium setting. Clarified samples were transferred to Eppendorf tubes and diluted five times with ChIP Dilution Buffer (0.01\% SDS, $1.1 \%$ Triton X-100, 1.2 mM EDTA, $16.7 \mathrm{mM}$ Tris- $\mathrm{HCl}$ pH 7.5, $167 \mathrm{mM} \mathrm{NaCl}$ ). Cells were precleared with $30 \mu \mathrm{L}$ of Protein $\mathrm{G}$ dynabeads (Life technology \#10004D) in rotation at $4^{\circ} \mathrm{C}$ for $1 \mathrm{~h}$. Supernatants were transferred into fresh tubes and antibody was added (3ug H3K27Ac antibody for 10 million cells) and incubated overnight at $4^{\circ} \mathrm{C}$. The next day $30 \mu \mathrm{L}$ of Protein $\mathrm{G}$ dynabeads were added to samples and rotated at $4^{\circ} \mathrm{C}$ for $2 \mathrm{~h}$. After bead capture, beads were washed three times each with low-salt wash buffer (0.1\% SDS, 1\% Triton X-100, 2 mM EDTA, 20 mM Tris$\mathrm{HCl} \mathrm{pH}$ 7.5, $150 \mathrm{mM} \mathrm{NaCl})$, high-salt wash buffer (0.1\% SDS, 1\% Triton X-100, 2 mM EDTA, $20 \mathrm{mM}$ Tris- $\mathrm{HCl} \mathrm{pH}$ 7.5, $500 \mathrm{mM} \mathrm{NaCl})$, and $\mathrm{LiCl}$ wash buffer (10 mM Tris- $\mathrm{HCl} \mathrm{pH}$ 7.5, 250 $\mathrm{mM} \mathrm{LiCl}, 1 \%$ NP-40, $1 \%$ sodium deoxycholate, $1 \mathrm{mM}$ EDTA). Samples were eluted with 150 $\mu \mathrm{L}$ of DNA elution buffer (50 mM sodium bicarbonate $\mathrm{pH} 8.0,1 \%$ SDS, freshly made) and incubated at $37^{\circ} \mathrm{C}$ for $30 \mathrm{~min}$ with rotation. Supernatant was transferred to a fresh tube and elution repeated with another $150 \mu \mathrm{L}$ elution buffer. $5 \mu \mathrm{L}$ of Proteinase K $(20 \mathrm{mg} / \mathrm{ml})$ (Thermo Fisher) were added to the $300 \mu \mathrm{L}$ reaction and samples were incubated overnight at $65^{\circ} \mathrm{C}$. Samples were purified with DNA Clean and Concentrator columns (Zymo Research) and eluted in $10 \mu \mathrm{L}$ of water. Post-ChIP DNA was quantified by Qubit (Thermo Fisher). $5 \mu \mathrm{L}$ of Streptavidin C-1 beads (Thermo Fisher) were washed with Tween Wash Buffer (5 mM Tris-HCl $\mathrm{pH} 7.5,0.5 \mathrm{mM}$ EDTA, $1 \mathrm{M} \mathrm{NaCl}, 0.05 \%$ Tween-20) then resuspended in $10 \mu \mathrm{L}$ of $2 \times$ biotin binding buffer (10 mM Tris- $\mathrm{HCl} \mathrm{pH} 7.5,1 \mathrm{mM}$ EDTA, $2 \mathrm{M} \mathrm{NaCl})$. Beads were added to the samples and incubated at room temperature for $15 \mathrm{~min}$ with shaking. After capture, beads were washed twice by adding $500 \mu \mathrm{L}$ of Tween Wash Buffer and incubated at $55^{\circ} \mathrm{C}$ for 2 min with shaking. Samples were then washed in $100 \mu \mathrm{L}$ of $1 \times$ TD Buffer $(2 \times$ TD Buffer is $20 \mathrm{mM}$ Tris$\mathrm{HCl} \mathrm{pH}$ 7.5, $10 \mathrm{mM}$ magnesium chloride, 20\% dimethylformamide). After washes, beads were resuspended in $25 \mu \mathrm{L}$ of $2 \times$ TD Buffer, Tn5 (for 50 ng of post-ChIP DNA we used $2.5 \mu \mathrm{L}$ of Tn5), and water to $50 \mu \mathrm{L}$. Tn5 amount was adjusted linearly for different amounts of post-ChIP 
DNA, with a maximum amount of $4 \mu \mathrm{L}$ of Tn5. Samples were incubated at $55^{\circ} \mathrm{C}$ with interval shaking for $10 \mathrm{~min}$. After removing the supernatant $50 \mathrm{mM}$ EDTA was added to samples and incubated with interval shaking at $50^{\circ} \mathrm{C}$ for $30 \mathrm{~min}$. Beads were then washed two times each in $50 \mathrm{mM}$ EDTA then Tween Wash Buffer at $55^{\circ} \mathrm{C}$ for $2 \mathrm{~min}$. Lastly, beads were washed in $10 \mathrm{mM}$ Tris before PCR amplification. Beads were resuspended in $25 \mu \mathrm{L}$ of Phusion HF $2 \times(\mathrm{New}$ England Biosciences), $1 \mu \mathrm{L}$ of each Nextera Ad1_noMX and Nextera Ad2.X at $12.5 \mu \mathrm{M}$, and 23 $\mu \mathrm{L}$ of water. The following PCR program was performed: $72^{\circ} \mathrm{C}$ for $5 \mathrm{~min}, 98^{\circ} \mathrm{C}$ for $1 \mathrm{~min}$, then cycle at $98^{\circ} \mathrm{C}$ for $15 \mathrm{~s}, 63^{\circ} \mathrm{C}$ for $30 \mathrm{~s}$, and $72^{\circ} \mathrm{C}$ for $1 \mathrm{~min}$ (cycle number was estimated based on the amount of material from the post-ChIP Qubit (approximately $50 \mathrm{ng}$ was run in six cycles, while $25 \mathrm{ng}$ was run in seven, $12.5 \mathrm{ng}$ was run in eight, etc.). Size selection was performed using two-sided size selection with the Ampure XP beads. After PCR, libraries were placed on a magnet and eluted into new tubes. $25 \mu \mathrm{L}$ of Ampure XP beads were added, and the supernatant was kept to capture fragments less than $700 \mathrm{bp}$. Supernatant was transferred to a new tube, and $15 \mu \mathrm{L}$ of fresh beads was added to capture fragments greater than $300 \mathrm{bp}$. After size selection, libraries were quantified with Qbit and sent for Bioanalyzer to check for the quality and final size of the library. Libraries were sequenced on an Illumina HiSeq4000 platform on PE50 mode.

\section{In vitro drug treatment}

CUTLL1 cells were treated with gamma secretase inhibitor (Compound E) purchased from Alexis Bioscience at a $1 \mu \mathrm{M}$ final concentration. Treatment was performed every 12 hours for 72 hours. THZ1 was purchased from Cayman Chemical (Catalog no: 9002215) and the cells were treated at $100 \mathrm{nM}$ final concentration every $12 \mathrm{~h}$ for $24 \mathrm{~h}$. For 5-azacytidine, the cells were treated with $100 \mathrm{nM}$ every day for 3 days $(72 \mathrm{~h})$.

\section{GRO-Seq and library preparation}

GRO-seq sequencing were performed in CUTLL1 cells treated with either DMSO or $\gamma \mathrm{SI}$ at $1 \mu \mathrm{M}$ for $72 \mathrm{~h}$. All experiments were performed in biological duplicates. Gro-seq sample preparation was performed as described previously ${ }^{6}$. Briefly, nuclei were isolated in swelling buffer (10 mM Tris- $\left.\mathrm{mCl} \mathrm{pH} 7.5,2 \mathrm{mM} \mathrm{MgCl}_{2}, 3 \mathrm{mM} \mathrm{CaCl}_{2}\right)$, lysed twice in lysis buffer (10 $\mathrm{mM}$ Tris- $\mathrm{HCl} \mathrm{pH} 7.5,2 \mathrm{mM} \mathrm{MgCl} 2,3 \mathrm{mM} \mathrm{CaCl}_{2}, 10 \%$ glycerol, $0.5 \% \mathrm{NP}-40$ ) and snap-frozen in freezing buffer (50 mM Tris $\mathrm{pH} 8.0,40 \%$ glycerol, $5 \mathrm{mM} \mathrm{MgCl}_{2}, 0.1 \mathrm{mM}$ EDTA), For run-on 
reaction, an equal volume of reaction buffer was added to thawed nuclei $(10 \mathrm{mM}$ Tris $\mathrm{pH}$ 8.0, $5 \mathrm{mM} \mathrm{MgCl} 2,300 \mathrm{mM} \mathrm{KCl}, 500 \mu \mathrm{M}$ ATP, $500 \mu \mathrm{M}$ GTP, $5 \mu \mathrm{M}$ CTP, $500 \mu \mathrm{M}$ BrUTP, $1 \mathrm{mM}$

dithiothreitol, $100 \mathrm{U} \mathrm{ml}^{-1}$ SuperaseIN, 1\% Sarkosyl), mixed and incubated at $30{ }^{\circ} \mathrm{C}$ for $5 \mathrm{~min}$. The reaction was stopped with Trizol reagent and RNA was phenol/chloroform extracted and ethanol precipitated. RNA was heated in fragmentation buffer $(40 \mathrm{mM}$ Tris $\mathrm{pH} 8.0,100 \mathrm{mM}$ $\mathrm{KCl}, 6.25 \mathrm{mM} \mathrm{MgCl} 2,1 \mathrm{mM}$ dithiothreitol), DNAse treated and purified using Zymo RNA Clean \& Concentrator (Zymo Research) using the >17nt protocol. Run-on RNA was immunoprecipitated using BSA-blocked BrDU beads (Santa Cruz) in binding buffer (SSPE 0.5X, $1 \mathrm{mM}$ EDTA, $0.05 \%$ Tween-20) for $1 \mathrm{~h}$ at $4{ }^{\circ} \mathrm{C}$, washed and eluted in elution buffer (5 mM Tris pH 7.5, $300 \mathrm{mM} \mathrm{NaCl}, 20 \mathrm{mM}$ dithiothreitol, $1 \mathrm{mM}$ EDTA, 1\% SDS) at $65^{\circ} \mathrm{C}$ for 20 min. Nascent RNA was further phenol/chloroform extracted and sequencing libraries were prepared.

\section{qPCR}

RNA was extracted using the RNeasy Mini Kit using Qiagen kit (Catalog no: 74106) folllowing manufacturer's guidelines. cDNA was generated using High Capacity RNA-to-cDNA kit from Life Technologies (Catalog no: 4387406) following manufacturer's guidelines. cDNA was used to perform qPCR using Light cycler 480 SYBR green I Master Mix from Roche Diagnostis (Catalog no: 04887352001). See Supplementary Table for primer sequences. The reactions were run in Roche Light cycler 480 II.

\section{Sanger sequencing of CTCF site in MYC locus}

Genomic DNA from CUTLL1, Jurkat and T-ALL1 were isolated using Qiagen DNeasy kit as per manufacturer's guidelines. Target locus was PCR amplified using Phusion High Fidelity PCR Master Mix (Thermo Fisher; Catolog no. F531S) using 100 ng genomic DNA as template. Primer sequences are listed in the table below. PCR product was purified using Qiagen PCR purification column and submitted for Sanger sequencing to Genewiz.

\section{Immunoblotting}

CUTLL1 cells treated with DMSO, $\gamma$ SI or THZ1 were pelleted and lysed using RIPA lysis and extraction buffer (Thermo Fisher, Catolog no: 89900). The lysates were boiled with Laemmli 
buffer, resolved by SDS-PAGE, transferred to PVDF membranes and proteins visualized by immunoblotting. c-MYC (D84C12) rabbit monoclonal antibody was purchased from Cell signaling and anti-actin antibody was purchased from Millipore (Catalog no. MAB1501R)

\section{CTCF targeting gRNA sequence}

The guide RNA target sequence is UCUACAACAUCUCCACCAUG. The guide RNA along with the tracer RNA was purchased as a synthetic guide RNA from Synthego with 2'-O-methyl 3' phosphorothioate modifications of the first and last three nucleotides.

\section{Editing of T cells}

Naïve T cells were activated with CD3/CD28 beads from Thermo Fisher Scientific (Catalog no: 11161D) for $48 \mathrm{~h}$. Following activation, the CD3/CD28 beads were magnetically removed and 2 million activated $\mathrm{T}$ cells were transfected by electrotransfer with either Cas9 $(1.5 \mu \mathrm{g})$ protein and $1 \mu \mathrm{g}$ guide RNA ribonucleoprotein complex or Cas9 $(1.5 \mu \mathrm{g})$ protein alone for every 200,000 cells using a Neon Transfection system at 1200 V, Width 40 and 1 pulse. Following electroporation, the cells were diluted into culture medium at $10^{6}$ cells per $\mathrm{ml}$. The electroporation step was repeated after $24 \mathrm{~h} .48 \mathrm{~h}$ post second transfection, genomic DNA was isolated. Target CTCF region was PCR amplified and subjected to Sanger sequencing. Editing efficiency was computed using the ICE computational program from Synthego.

\section{High-throughput 3D DNA-FISH}

\section{Generation of FISH probes}

Custom FISH probes targeting MYC promoter and enhancer were designed using the SureDesign custom oligo design tool from Agilent with homology to the regions of interest mined from the hg19 genome build using the default parameters of the SureDesign tool. The MYC promoter probe library targeted a $60 \mathrm{~Kb}$ region centered around the promoter whereas the enhancer probe library targeted a $100 \mathrm{~Kb}$ region targeting the center enhancer element of the MYC super-enhancer cluster. 


\section{D-FISH experimental protocol}

3D-FISH was performed using the Dako FISH Histology accessory kit from Agilent (Catlog no: K579911-5) as per manufacturer guidelines. Briefly, 200,000 cells were cytospun to poly-Llysine-treated glass slides at $1200 \mathrm{rpm}$ for $5 \mathrm{~min}$. Cells were subsequently fixed for 10 minutes with $4 \%$ formaldehyde in PBS at room temperature (RT), followed by membrane permeabilization with $0.5 \%$ Triton X-100 in PBS for 20 minutes at RT. The slides were washed once in 1X PBS followed by RNAse treatment (100 $\mu \mathrm{g} / \mathrm{ml}$ RNAse A in 2X SSC buffer). The cells were then wash with 2X SSC and dehydrated through alcohol series: 2X 100\% ethanol and 2X 70\% ethanol, 2 minutes each at RT. The slides were washed with 1X Dako Wash buffer for 5 minutes at RT and treated with 1X Dako pre-treatment solution at $98{ }^{\circ} \mathrm{C}$ for 2 minutes and allowed to cool down for 15 minutes at RT. Following pre-treatment, the slides were washed twice with 1X Dako Wash buffer for 3 minutes each at RT. Then the slides were treated with cold pepsin at $37^{\circ} \mathrm{C}$ for 2 minutes followed by two washes with $1 \mathrm{X}$ Dako Wash buffer for 3 minutes each at RT. Then the slides were dehydrated through a series of ethanol washes $70 \%$ ethanol, $80 \%$ ethanol and 100\% ethanol, 2 minutes each at RT. Following the ethanol washes, the slides were air dried and set up for probe hybridization. For each slide $1 \mu 1$ of each probe mixed with $9 \mu \mathrm{l}$ of IQFISH Fast Hybridization buffer were added, covered with a coverslip and sealed with rubber cement. The slides were incubated at $80^{\circ} \mathrm{C}$ in a heat block for 10 minutes followed by 90 -minute incubation in a hot air oven set at $45^{\circ} \mathrm{C}$ in dark. Following hybridization, the rubber cement was removed and the slides were washed with 1X Dako stringent wash buffer for 5 minutes at RT immediately followed by a second wash with 1X Dako stringent wash buffer for 10 minutes at $56^{\circ} \mathrm{C}$. The stringent washes were followed with two washes of $1 \mathrm{X}$ Dako Wash buffer for 3 minutes each at RT. The slides were then dehydrated through a series of ethanol washes 70\% ethanol, 80\% ethanol and 100\% ethanol, 2 minutes each at RT, air dried and mounted with coverslips using immune-mount with DAPI stain.

\section{D FISH quantification of MYC enhancer-promoter distances}

To quantify enhancer-promoter distances from DNA FISH data, we first used AirLocalize (Lionnet et al, Nature Methods 2011) to automatically detect spots in enhancer and promoter channels and estimated their position with subpixel resolution. For enhancers (red channel), the detection parameters were set to $\sigma x y=2.4621, \sigma z=1.1768$ pixels and the intensity threshold to 
5000 counts (typical voxel sizes: $73 \mathrm{~nm}$ in xy, $360 \mathrm{~nm}$ in z). For promoters (green channel), the detection parameters were set to $\sigma \mathrm{xy}=2.8801, \sigma \mathrm{z}=1.1776$ pixels and the intensity threshold to 4000 counts. Then we filtered the spots by eliminating spots located outside the nucleus or with integrated intensity lower than $1.5 \mathrm{e} 6$ counts in red or $0.75 \mathrm{e} 6$ counts in green. Next, we computed and plotted the histograms of the distance matrix between spots in the red and green channels. For a perfectly aligned and corrected system, the means of the $\mathrm{dx}$, dy and dz histograms should be 0 in all three directions for there is no reason for enhancers to prefer one relative orientation to promoters than others. We therefore corrected the offsets of the two channels by subtracting the means of the $\mathrm{dx}, \mathrm{dy}$ and $\mathrm{dz}$ histograms from individual coordinate differences. After correction, we computed the distance matrix again. In the distance matrix, we found pairs of spots that are the nearest neighbors to each other, mutual nearest neighbors, which we defined as pairs of enhancers and promoters and built the matrix of their distances. Finally, we plotted cumulative probability distributions of enhancer-promoter distances in the different conditions $(\mathrm{N}=30,23$ and 16 image stacks for the CUTTL1-DMSO, CUTTL1-THZ1 and T cells respectively; a typical image contains 10-20 nuclei; probe-pairs T cells $=$ 993, probe-pairs CUTLL1 DMSO = 2001, probe-pairs CUTLL1 THZ1 = 1308). Results were robust to changes in bin size, subsets of images analyzed, or slight changes in imaging conditions, or considering all nearest neighbors rather than only mutual nearest neighbors. Significance for differential co-localization was derived using a Kolmogorov-Smirnow test.

\section{T cell donor Information}

$\begin{array}{lll} & \text { Age (Years) } & \text { Sex } \\ \text { T_cell_donor } & 60 & \text { Male } \\ \text { T_cell_donor } & 41 & \text { Male } \\ \text { T_cell_donor } & 63 & \text { Male } \\ \text { T_cell_donor } & 38 & \text { Male } \\ \text { T_cell_donor } & 19 & \text { Male } \\ \text { T_cell_donor } & 60 & \text { Male }\end{array}$




\section{Bioinformatics analysis:}

\section{Hi-C analysis}

In-situ Hi-C datasets were analyzed with the HiC-bench platform ${ }^{7}$. In short, both datatypes were aligned against the human reference genome (GRCh37/hg19) by bowtie2 (version 2.3.1) ${ }^{8}$ with mostly default parameters (specific settings: --very-sensitive-local --local). For Hi-C, aligned reads were filtered by the GenomicTools ${ }^{9}$ tools-hic filter command (integrated in HiC-bench), which discards multi-mapped reads ("multihit"), read-pairs with only one mappable read ("single sided"), duplicated read-pairs (“ds.duplicate"), read-pairs with a low mapping quality of MAPQ $<20$, read-pairs resulting from self-ligated fragments (together called "ds.filtered") and shortrange interactions resulting from read-pairs aligning within $25 \mathrm{~kb}$ (“ds.too.short"). The reads used for downstream analyses are all accepted intra-chromosomal read-pairs ("ds.accecpted intra"), which were consistently above $25 \%$ across all Hi-C samples. The absolute number of accepted intra-chromosomal read-pairs varied between $\sim 40$ and $\sim 120$ million, which in all cases was sufficient to call topologically associated domains (TADs). Interaction matrices for each chromosome separately were created by the $\mathrm{HiC}$-bench platform at a $40 \mathrm{~kb}$ resolution. Filtered read counts were normalized by a method called "iterative correction and eigenvector decomposition" (ICE) ${ }^{10}$. To account for variances of read counts of more distant loci, which tend to be less covered the further distant the interacting loci are apart in the genome, we performed distance normalization for each matrix as recently described ${ }^{11}$.

TADs were called using the algorithm developed within hic-bench ${ }^{7}$ setting the insulating window to $500 \mathrm{~kb}$. The matrix-wide stratum-adjusted correlation score (SCC) was calculated using HiC-Rep ${ }^{12}$ for each possible pair-wise comparisons of all $14 \mathrm{Hi}-\mathrm{C}$ samples. The SCC was first calculated for each pair of chromosome matrices for any possible pair-wise comparison. The final score for a sample-comparison was calculated as the average of all its chromosome scores. Principal Component Analysis (PCA) on Hi-C datasets was performed in R (prcomp, with scale=TRUE and center=TRUE) using the genome-wide Hi-C "ratio" insulation scores for 500kb windows, as defined in Lazaris et al. ${ }^{7}$. Unsupervised clustering on hic-ratio insulation scores was performed using the $\mathrm{R}$ package Mclust version 5.3 (https://cran.rproject.org/web/packages/mclust/index.html), and both EII and VII models found three clusters to be the optimal separation of samples. For visualization of $\mathrm{Hi}-\mathrm{C}$ data, we created heatmaps for regions of interest using the normalized contact matrices. Heatmaps were generated with the $\mathrm{R}$ 
function image, and color scale was set to the highest normalized score seen in any sample for the particular window. Fold-change heatmaps were generated by calculating the log2 foldchange for each matrix bin vs. T cell 1 sample.

\section{CTCF \& H3K27ac ChIP-Seq analysis}

ChIP-Seq datasets were analyzed with the HiC-bench platform ${ }^{7}$. The ChIP-Seq aligned reads were further filtered by discarding reads with low mapping quality (MAPQ < 20) and duplicated reads using picard-tools (https://github.com/broadinstitute/picard). The remaining reads were analyzed by applying the peak-calling algorithm MACS2 (version 2.0.1) ${ }^{13}$ with input as control (option -c) wherever applicable. Binding of transcription factor CTCF was determined from narrow-peak calls, while histone-marks were determined from broad-peak calls (option --broad). For differential binding affinity analysis, we ran the $\mathrm{R}$ Bioconductor package diffBind with default parameters, which outputs p-value, false-discovery rate and fold-changes of binding affinity for all identified peaks from either sample of any possible pair-wise comparison. For normalization during diffBind, we used the option "method=DBA_EDGER". For visualization, we generated bigwig tracks (with the help of bedtools version 2.27.1) as fold-enrichment combining all replicates of the actual sample over input wherever applicable using the MACS2 bdgcmp function (with “-m FE”). All bigwig tracks shown were created with IGV (version 2.3.83). CTCF orientation for canonical CTCF binding sites depicted in all tracks with CTCF ChIP-Seq was generated by PWMScan ${ }^{14}$ (database JASPAR CORE vertebrates; filtered by pvalue $<1 \mathrm{E}-5)$. Differential binding heatmaps and peak signal quantification were generated with deeptools (version 2.3.3) ${ }^{15}$ and visualized the $2.5 \mathrm{~kb}$ up- and downstream of the peak-summit.

\section{RNA-Seq}

RNA-Seq reads were aligned against the human reference genome (GRCh37/hg19) using the STAR aligner (version 2.5.0c) ${ }^{16}$ mostly with default parameters, discarding all non-uniquely aligned reads. Duplicated reads were discarded using picard-tools. For read counting per gene, we used bamutils count of the ngsutils package (version 0.5 .7$)^{17}$ on gene annotations from Ensembl V75 in a stranded manner (options -uniq -multiple complete -library RF). Downstream processing was performed in $\mathrm{R}$ with the Bioconductor package edge $\mathrm{R}$ (version 3.14 .0$)^{18}$ on stranded gene counts, normalizing for intra- and inter-sample variances (edgeR functions 
calcNormFactors and estimateTagwiseDisp), resulting in counts-per million (CPM) per annotated gene. For cell line data with multiple replicates (CUTLL1 n=3, Jurkat n=2), CPM values were averaged. Differential expression analysis was performed per condition (leukemia vs. normal T cells) with edgeR functions glmQLFit and glmQLFTest.

\section{GRO-Seq}

GRO-Seq reads were aligned against the human reference sequence GRCh37/hg19 using bowtie (version 1.0.0) ${ }^{8}$. All aligned reads were filtered for unique alignment positions (MAPQ > 20). Next, the remaining reads were counted in a stranded manner per annotated gene in Ensembl Genes V75 using bamutils count of the ngsutils package (version 0.5.7; options -uniq -multiple complete -library RF) ${ }^{17}$. We performed normalization using edge ${ }^{18}$ (version 3.14.0; functions calcNormFactors and estimateTagwiseDisp), resulting in counts per millions (CPM) per gene after averaging data from replicates. For visualization, we created bigwig tracks per genomic strand using bedtools coverage (2.27.1) after normalizing for sequencing depth and fragment length of 250bp (bedtools coverage option -fs 250). All tracks were visualized with IGV (version 2.3.83).

\section{C-Seq}

4C-Seq reads were processed similarly as described in ${ }^{19,20}$. First, a reduced genome reference was created for the human reference genome (GRCh37/hg19) by only considering unique sequence fragments from the reference genome sequence that are adjacent to the restriction sites of the restriction enzyme (DpnII) used during the 4C protocol (following the 4C-ker pipeline ${ }^{19}$ ). All reads were aligned against this reduced genome reference by bowtie (version 1.0.0) ${ }^{8}$, only considering uniquely aligned reads. All self-ligated and undigested fragments were removed (following the 4C-ker pipeline). We further validated that all samples had $>0.5$ million mapped reads and $>0.5$ cis/trans ratio of mapped reads ${ }^{20}$. Next, we defined successive overlapping windows of different resolutions (10kb and 40kb), and all adjacent windows are overlapping by $90 \%$ of their length ( $9 \mathrm{~kb}$ and $36 \mathrm{~kb}$ respectively). We counted uniquely mapped reads for each window per sample and performed normalization with edgeR (leading to CPM per window). This accomplishes a smoothed signal across samples for different sizes of regions to be plotted (approx. 300kb in Figures 5E, 5F, S10E and 6E using 10kb resolution and $\sim 2 \mathrm{MB}$ in Figures 4C, 
S7, S8E, S10A, 6D and S11E using 40kb resolution). Data from biological replicates were averaged after normalization for visualization. Differential interactions were identified with edgeR (version 3.14.0) functions glmQLFit and glmQLFTest, and $\log 10$ (p-value) is shown on the negative $\mathrm{y}$-axis of all $4 \mathrm{C}$ plots as indicator for the most significant changes. We have not performed multiple testing correction, as each data-point is dependent due to overlapping windows, and would thus potentially lead to a too stringent correction. Quantifications were calculated for the highest single peak (at 10kb resolution for LUNAR1, APCDD1, IKZF2; at $40 \mathrm{~kb}$ resolution for MYC) within depicted enhancers/promoters in the respective Figures by grey boxes. Normalized 4C signals, as calculated by cpm-function within edgeR, were further normalized against the average control replicates, and shown in percent. Specific p-values shown in Figures were also taken for the same 10kb/40kb bin showing highest 4C signal within respective enhancer/promoter.

\section{Compartment analysis}

Compartment calling was performed using the filtered $\mathrm{Hi}-\mathrm{C}$ reads of the hic-bench pipeline for all $14 \mathrm{Hi}-\mathrm{C}$ samples individually. The "c-score tool" ${ }^{21}$ was used to determine the A and B compartments at $100 \mathrm{~kb}$ windows, using information on active chromatin from $\mathrm{H} 3 \mathrm{~K} 27 \mathrm{ac}$ ChIPSeq in T cells, CUTLL1 (for T-ALL) and Loucy (for ETP-ALL) to assign A/B to resulting compartment scores. Windows with missing c-score values for at least one sample are removed from the analysis. P-values were calculated using an unpaired two-sided t-test to determine the statistical significance of compartment shifts for the following comparisons: T-ALL vs T cells, ETP-ALL vs T cells and ETP-ALL vs T-ALL samples. After p-value calculation, the mean cscore for all T-ALL, all ETP-TALL and all T cell samples were calculated. Compartment shifts were determined as "A to A" when the mean c-score values for both conditions are $>0.1$, "B to B" shift if the mean c-score value for both conditions is < -0.1 , and "A to B"/"B to A" shift if the mean c-score value of one condition is $<-0.1$ and $>0.1$ for the other condition (p-value $<0.1$ ). Unique compartment shifts for either T-ALL or ETP-ALL were identified by combining the results of the above three comparisons. More specifically, an "A to B" shift is considered T-ALL specific if it is identified as an "A to B" shift in the T-ALL vs T cell comparison, but as a "B to A" event in the ETP-ALL vs T-ALL comparison. A "B to A" shift is considered T-ALL specific when it is identified as a "B to A" shift in the T-ALL vs T cell comparison, but as an "A to B" 
shift for the ETP-ALL vs T-ALL comparison. Similarly, an "A to B" shift is ETP-ALL specific, when it is found as an "A to B" shift for the ETP-ALL vs T cell comparison and an "A to B" shift in the ETP-ALL vs T-ALL comparison; a "B to A" shift is ETP-ALL specific when it is identified as a "B to A" shift in both ETP-ALL vs T cell and ETP-ALL vs T-ALL comparisons.

\section{Differential TAD activity and data integration}

To identify TADs of differential intra-TAD activity, we developed an algorithm to detect statistically significant overall changes between samples of any two conditions (e.g. T-ALL vs. T cells). Firstly, we identified TADs that are common in both conditions. This was done by only considering TADs whose boundaries on either side of the TAD are as close as three bins between the two samples (i.e. $120 \mathrm{~kb}$ in a $40 \mathrm{~kb}$ resolution), setting the boundaries of the common TAD to those which yield the largest TAD. We also set a minimum TAD length to 10 bins $(400 \mathrm{~kb})$. We further removed TADs that fall in the B compartment in both conditions by at least $75 \%$ of the genomic TAD area, to avoid minor changes in TAD activity within highly repressed chromatin. This set of common TADs between any two conditions $c_{1}$ and $c_{2}$ is denoted as $T$. For each interaction bin, we averaged the Hi-C matrix score across conditions (i.e. all $\mathrm{T}$ cell, $\mathrm{T}$-ALL or ETP-ALL samples). Next, we performed a paired two-sided t-test on each single interaction bin within each common TAD between the average Hi-C matrix values per condition and calculated the $\log 2$ fold-change between the average scores of all interaction intensities within such TADs between the two samples:

$$
\left.T A D \text { activity change }(t)=\log 2\left(\frac{\sum_{i \in I_{t}} c_{2_{i}}}{\# I_{t}}\right) /\left(\frac{\sum_{i \in I_{t}} c_{1_{i}}}{\# I_{t}}\right)\right)
$$

for each $t \in T$, and $I_{t}$ being all intra-TAD interactions for TAD $t$.

We also applied multiple testing correction by calculating the false-discovery rate per common TAD (using the R function p.adjust with method="fdr"). For downstream analyses, we filtered common TADs as differentially active by setting the FDR $<0.1$ and absolute $\log 2$ fold-change $>$ 0.58. As a negative control group, we defined stable TADs of stable high activity by filtering for TADs with an absolute $\log 2$ fold-change $<0.1$ and average TAD activity within the top $50 \%$ quantile of all TAD activity scores. For downstream CTCF occupancy integration, we extended the TAD boundary for each such identified TAD by 2 bins $(80 \mathrm{~kb})$ on either side of the boundary accounting for false boundary calls. Changes in CTCF occupancy within these boundaries were 
defined as the sum of all their $\log 2$ FCs taken from the diffBind output, matching the equivalent comparison of T cells vs. T-ALL. Significant changes in global CTCF occupancy within such boundaries were calculated using a one-sided t-test on logFCs from each group (i.e. higher or lower activity in leukemia samples) vs. stable TADs, following the hypothesis of a positive correlation between CTCF binding and TAD boundary strength / TAD activity as recently reported ${ }^{22}$. Genes (Ensembl V75 annotations; only protein-coding, processed transcripts and lincRNAs with FPKM > 1) were integrated if their promoters were falling within the TADs, extending each TAD by 2 bins $(80 \mathrm{~kb})$ to either side accounting for inaccurate boundary calls. For each gene, we took the $\log 2 \mathrm{FC}$ from the edgeR output for the respective comparison (T cell vs T-ALL or ETP-ALL vs T-ALL). Significance in global changes of RNA expression was calculated as a one-sided t-test on $\operatorname{logFC}$ from each group (i.e. higher or lower activity in leukemia samples) vs. stable TADs, following the hypothesis of a positive correlation between TAD activity and gene expression.

\section{Super-enhancer calling and integration}

For T cell and CUTLL1 H3K27ac ChIP-Seq data, we applied our standard ChIP-Seq analysis pipeline (https://github.com/NYU-BFX/hic-bench), as described above in detail. Next, we ran ROSE $^{23}$ with default parameters to define super-enhancers. For each dataset, we have excluded common super-enhancers defined as super-enhancers from both cell-types overlapping by at least $1 \mathrm{bp}$ on the genomic coordinates in order to define cell-type specific super-enhancers. We overlapped the remaining cell-type specific super-enhancers with differential active TADs if the overlapping genomic coordinates were larger than $1 \mathrm{bp}$. Enrichment score ES of super-enhancers defined as observed over expected overlap was calculated as follows:

$$
E S=\frac{O V}{\frac{D A \_T A D S * S E \_T A D S}{T}}
$$

with $S E_{-} T A D S$ being all TADs containing at least one super-enhancer, DA_TADs being all differentially active TADs, $O V$ being the intersection of $S E_{-} T A D S$ and $D A_{-} T A D S$ and $T$ being all TADs the analysis was performed on. Statistically significant enrichment against background $\left(S E_{-} T A D S\right)$ was determined using a two-sided Fisher exact test. 


\section{Compartment shifts within differentially active TADs}

We have overlapped compartment information with the differentially active TADs for the TALL vs T cell comparison to determine potential compartment shifts within the genomic area of each TAD. Therefore, we have defined "A to B", "B to A", "A to A" and "B to B" shifts as described above. To this end, the length of each TAD was determined and the numbers of compartment shifts from any of the previous categories overlapping each TAD were calculated. Then, the percentage of overlap for each TAD was calculated regarding the four compartment shift categories and the average overlap of each category across all TAD categories is shown.

\section{TAD boundary insulation alterations and differential CTCF integration}

We sought to detect more complex changes in chromatin architecture by defining TAD boundary insulation alterations. We separated those into losses and gains of TAD boundaries between normal $\mathrm{T}$ cells and leukemia (as depicted in Figure 3A). The analysis was performed in a twostep approach, differentiating between TAD boundary loss and TAD boundary gain as changes resulting from lost versus novel TAD boundaries from the perspective of the leukemia samples, respectively. We thus performed the analysis of identifying insulation changes based on adjacent $\mathrm{T}$ cell TADs (yielding TAD boundary losses) and vice versa on adjacent leukemia TADs (yielding TAD boundary gains). Thus, for each pair of adjacent TADs for either T cell or T-ALL TAD calls, we determined the interaction strengths of all inter-TAD interactions and intra-TAD interactions for both the two adjacent TADs. The TAD boundary insulation alteration score BIC for each pair of adjacent TADs was calculated as BIC = inter-TAD interactions * max(intraTAD interactions). To select the strongest outliers of this analysis as TAD boundary alterations, we took the top 5\% quantile of all BIC scores between T-ALL and normal T cells. To determine whether these outliers are significant, we performed the same analysis between all three normal $\mathrm{T}$ cell donors, applying the same threshold taken from the $\mathrm{T}$ cell vs. T-ALL comparison, assuming that there are no severe TAD boundary alterations between any two normal controls. This yielded on average 12 (TAD boundary loss) and 17 (TAD boundary gain) outliers for all three pair-wise comparisons of normal controls, thus we achieved controlling for an average FDR $\sim 10.77 \%$ in $\mathrm{T}$ cells vs. T-ALL under the assumption of no boundary insulation changes between normal T cells. For interesting loci, we manually integrated CTCF occupancy and RNA expression changes. 


\section{Genome-wide detection of enhancer activity changes in $\gamma$ SI/THZ1 treated samples}

All detected H3K27ac peaks from ChIP-Seq were first overlaid with promoters of annotated genes taken from Ensembl Genes V75. All peaks with a distance of more than $1 \mathrm{~kb}$ from an annotated promoter (measured from the peak-boundaries) were considered enhancers. Then, we created sets of stable/constant, loss and gain of enhancers in CUTLL1 after $\gamma$ SI or THZ1 treatment using diffBind ${ }^{24}$ on H3K27ac ChIP-Seq. For stable enhancers, we filtered all peaks with $\operatorname{abs}(\log \mathrm{FC})<0.2$; for reduced/loss of enhancer activity, we filtered all peaks with $\operatorname{logFC}<-$ $1.0 />1.0$ and FDR $<0.05$. For $\gamma$ SI-treatment data, we further overlapped all three groups with dynamic NOTCH1-binding sites taken from Wang et al. ${ }^{25}$. Enrichment scores (observed over expected) were calculated similarly as described above, using a two-sided Fisher's exact test for significance calculation.

\section{Differential binding analysis using LOLA}

In order to define potential co-factors of enhancer/looping activity in $\gamma$ SI-sensitive and insensitive enhancers (Suppl. Figure 9F), we used LOLA ${ }^{26}$. To this end, we downloaded the LOLA database (http://databio.org/regiondb) and kept ChIP-Seq data from T-ALL related cell lines (Jurkat, CUTLL1 or HPB-ALL), that displayed at least 3000 peaks. We are representing the results as percent overlap between ChIP-Seq peaks and $\gamma$ SI-sensitive / insensitive genomic locations. Statistics for differences between $\gamma$ SI-sensitive and insensitive enhancers was calculated using a two-sided Fisher exact test.

\section{HiChIP data analysis and loop calling}

H3K27ac HiChIP data in CUTLL1 was processed with the hic-bench platform similarly as described above for Hi-C data. We have used output of filtered/accepted intra-chromosomal reads, and performed mango ${ }^{27}$ to identify significant loops at a $40 \mathrm{~kb}$ resolution. The trajectories of each matrix bin of the HiChIP data onto both anchors on the diagonal were overlaid with peaks identified from H3K27ac ChIP-Seq in CUTLL1, requiring a minimal overlap of $1 \mathrm{bp}$ between a HiChIP-bin and a ChIP-peak. Only loops that were supported by a ChIP-peak in at least one anchor were kept for further analyses. We then applied sequencing-depth normalization (CPM) per replicate followed by a statistical approach described in mango, which employs a binomial test in each diagonal of the counts-matrix up to a maximum distance of $2 \mathrm{Mb}$. High- 
confidence HiChIP loops were identified by FDR $<0.1$ and requiring a minimum CPM > 5 per loop. We have only kept loops that contain an H3K27ac peak outside any annotated promoter in one anchor and an annotated promoter in the other anchor, thus defining promoter-enhancer loops for downstream Hi-C integration analyses.

\section{Hi-C analysis for $\gamma$ SI/THZ1 treated cells using HiChIP defined enhancer-promoter interactions}

Next, to investigate the involvement of changes in enhancer H3K27ac signal in nearby gene expression and loop formation upon $\gamma \mathrm{SI} / \mathrm{THZ} 1$ treatment in CUTLL1, we integrated Hi-C data with promoter-enhancer loops. To this end, we first identified candidate interactions of promoterenhancer pairs using loop calling from H3K27ac HiChIP data in CUTLL1, as described above. We further took these specific promoter-enhancer pairs and calculated changes in Hi-C connectivity, using the normalized contact matrices at $40 \mathrm{~kb}$ resolution. We calculated $\log 2$ foldchanges between DMSO and $\gamma \mathrm{SI} / \mathrm{THZ1}$ treatment matrices after averaging $\mathrm{Hi}-\mathrm{C}$ matrices across replicates in each condition. Global loss/gain of interactions upon $\gamma \mathrm{SI} / \mathrm{THZ} 1$ treatment was depicted by a one-sided t-test comparing all groups vs. the stable H3K27ac group, following the hypothesis of a positive correlation between promoter-enhancer looping and enhancer activity.

\section{Integration of GRO-Seq data with findings from combined H3K27ac ChIP and HiChIP analysis}

For all genes connected with nearby differential/stable enhancers (categorized by ChIP-Seq as described above) detected from HiChIP, we investigated expression of such genes before treatment, after treatment and after 1, 2, 3, 4, 5, 6, and 10 hours post drug "wash off". We are representing the median FPKM across all genes (FPKM > 1) of a respective enhancer-promoter loop category per time-point. The genome-wide trend of reduced expression for specific H3K27ac categories was determined by a one-sided t-test comparing expression with all genes within stable H3K27ac enhancer-loops, following the hypothesis of a positive correlation between expression changes and looping/enhancer activity. 


\section{WGS analysis and integration with TADs and CTCF binding}

Whole-genome sequencing and subsequent data analysis in primary T-ALL samples was performed by GeneWiz (https://www.genewiz.com/). In short, copy number variants were called using Canvas version 1.3.1 and SVs were called using Manta version 0.28.0. Results of $\mathrm{CNVs}$ /tandem duplications, other SVs or SNVs were overlapped with genomic areas of (differentially active) TADs or TAD boundaries expanded by 1 bin $(40 \mathrm{~kb})$ in each direction using bedtools intersect and a minimum overlap of 1bp. Overlap of SVs or SNVs with CTCF binding information was performed by first overlapping differential CTCF peak calls with CTCF motif information derived from PWMScan (database JASPAR CORE vertebrates; filtered by pvalue $<1 \mathrm{E}-5$ ), and then with SVs or SNVs using bedtools intersect and a minimum overlap of 1bp. Significance of overlaps was calculated using two-sided Fisher exact test.

\section{CNV calling from genome-wide $\mathrm{Hi}-\mathrm{C}$ data}

We have used HiCnv ${ }^{28}$ in order to detect copy-number variations from all $14 \mathrm{Hi}-\mathrm{C}$ samples conducted within this study (excluding cell line data with drug treatments). We have used default parameters, with frag_limit=150 (in the script run_HiCnv.pl) as suggested by authors for Arima Hi-C (combination of frequent 4bp-cutting enzymes) and frag_limit=500 (in the script run_HiCnv.pl) as suggested by authors for Hi-C using HindIII (infrequent 6bp-cutting enzyme). Resulting copy-number variant bins were merged if adjacent genomic bins had the same predicted copy number variant score, and CNVs were called if any such merged bin had a detected CNV of $>3.5$ (CNV gain) or $<1.25$ (CNV loss).

\section{Primer sequences}

$\begin{array}{ll}\text { qPCR primers } & \\ \text { Actin_F } & \text { ACCACACCTTCTACAATGAGC } \\ \text { Actin_R } & \text { GATAGCACAGCCTGGATAGC } \\ \text { C-MYC_F } & \text { CTTCTCTCCGTCCTCGGATTCT } \\ \text { C-MYC_R } & \text { GAAGGTGATCCAGACTCTGACCTT } \\ \text { LUNAR1_F } & \text { GGCTAAGGGAGTCCAATCTTCC } \\ \text { LUNAR1_R } & \text { CAAGCTTGGGTCTGTCTGGT } \\ \text { APCDD1_F } & \text { CTGATGCCACCCAGAGGATG } \\ \text { APCDD1_R } & \text { AGATGATCCGACAGGCGATG } \\ \text { CTCF_ChIP_F } & \text { AGAGTTCAAAGGGACTGTCAAGGT }\end{array}$




\title{
CTCF_ChIP_R AGAAAAATACTGGTTGACTTGGGAGGT
}

\section{C primers}

MYC 4 C _ F

MYC_4C_R

LUNAR1_4C_F

LUNAR1_4C_R

APCDD1_4C_F

APCDD1_4C_R

IKZF2_F

IKZF2_R
TCTCCCTGGGACTCTTGATC

GTCTGTTTAGCCCTGAGATG

TGGGTAGAGTAAGACAGATC

GTGCTGTCTGTATAGGGCTC

TAACATCCGAGGAGGTGATC

TAGAGGCGAGGACATTTCTA

TTAAAGCTGTTAAGTGGATC

CACTGTGTATTTCCAATAGCC

\section{Genomic PCR}

\begin{abstract}
CTCF_site_MYC_locus_F TGAGCTCTGACTTGGTGACA
CTCF_site_MYC_locus_R TGATCAGAGTTAGGAACACATTCA
\end{abstract}

\section{References}

1 Pitt, L. A. et al. CXCL12-Producing Vascular Endothelial Niches Control Acute T Cell Leukemia Maintenance. Cancer Cell 27, 755-768, doi:10.1016/j.ccell.2015.05.002 (2015).

2 Rao, S. S. P. et al. A 3D Map of the human genome at kilobase resolution reveals principles of chromatin looping. Cell 159, doi:10.1016/j.cell.2014.11.021 (2014).

3 Ntziachristos, P. et al. Genetic inactivation of the polycomb repressive complex 2 in $\mathrm{T}$ cell acute lymphoblastic leukemia. Nat Med 18, 298-303 (2012).

4 Narendra, V. et al. CTCF establishes discrete functional chromatin domains at the Hox clusters during differentiation. Science (New York, N.Y.) 347, 1017-1021, doi:10.1126/science.1262088 (2015).

5 Mumbach, M. R. et al. HiChIP: efficient and sensitive analysis of protein-directed genome architecture. Nature methods 13, 919, doi:10.1038/nmeth.3999

https://www.nature.com/articles/nmeth.3999\#supplementary-information (2016).

6 Strikoudis, A. et al. Regulation of transcriptional elongation in pluripotency and cell differentiation by the PHD-finger protein Phf5a. Nature cell biology 18, 1127-1138, doi:10.1038/ncb3424 (2016).

7 Lazaris, C., Kelly, S., Ntziachristos, P., Aifantis, I. \& Tsirigos, A. HiC-bench: comprehensive and reproducible $\mathrm{Hi}-\mathrm{C}$ data analysis designed for parameter exploration and benchmarking. BMC Genomics 18, 22, doi:10.1186/s12864-016-3387-6 (2017).

8 Langmead, B. \& Salzberg, S. L. Fast gapped-read alignment with Bowtie 2. Nature methods 9, 357-359, doi:10.1038/nmeth.1923 (2012).

9 Tsirigos, A., Haiminen, N., Bilal, E. \& Utro, F. GenomicTools: a computational platform for developing high-throughput analytics in genomics. Bioinformatics 28, 282-283, doi:10.1093/bioinformatics/btr646 (2012). 
10 Imakaev, M. et al. Iterative correction of Hi-C data reveals hallmarks of chromosome organization. Nat Meth 9, 999-1003,

doi:http://www.nature.com/nmeth/journal/v9/n10/abs/nmeth.2148.html\#supplementaryinformation (2012).

11 Gong, Y. et al. Stratification of TAD boundaries reveals preferential insulation of superenhancers by strong boundaries. Nature communications 9, 542, doi:10.1038/s41467018-03017-1 (2018).

12 Yang, T. et al. HiCRep: assessing the reproducibility of Hi-C data using a stratumadjusted correlation coefficient. Genome Research 27, 1939-1949, doi:10.1101/gr.220640.117 (2017).

13 Zhang, Y. et al. Model-based analysis of ChIP-Seq (MACS). Genome Biol 9, R137, doi:10.1186/gb-2008-9-9-r137 (2008).

14 Ambrosini, G., Groux, R. \& Bucher, P. PWMScan: a fast tool for scanning entire genomes with a position-specific weight matrix. Bioinformatics, doi:10.1093/bioinformatics/bty127 (2018).

15 Ramirez, F. et al. deepTools2: a next generation web server for deep-sequencing data analysis. Nucleic Acids Res 44, W160-165, doi:10.1093/nar/gkw257 (2016).

16 Dobin, A. et al. STAR: ultrafast universal RNA-seq aligner. Bioinformatics 29, 15-21, doi:10.1093/bioinformatics/bts635 (2013).

17 Breese, M. R. \& Liu, Y. NGSUtils: a software suite for analyzing and manipulating nextgeneration sequencing datasets. Bioinformatics 29, 494-496, doi:10.1093/bioinformatics/bts731 (2013).

18 Robinson, M. D., McCarthy, D. J. \& Smyth, G. K. edgeR: a Bioconductor package for differential expression analysis of digital gene expression data. Bioinformatics 26, 139140, doi:10.1093/bioinformatics/btp616 (2010).

19 Raviram, R. et al. 4C-ker: A Method to Reproducibly Identify Genome-Wide Interactions Captured by 4C-Seq Experiments. PLoS computational biology 12, e1004780, doi:10.1371/journal.pcbi.1004780 (2016).

20 Rocha, P. P. et al. A Damage-Independent Role for 53BP1 that Impacts Break Order and Igh Architecture during Class Switch Recombination. Cell Rep 16, 48-55, doi:10.1016/j.celrep.2016.05.073 (2016).

21 Zheng, X. \& Zheng, Y. CscoreTool: fast Hi-C compartment analysis at high resolution. Bioinformatics 34, 1568-1570, doi:10.1093/bioinformatics/btx802 (2018).

22 Gong, Y. et al. Stratification of TAD boundaries reveals preferential insulation of superenhancers by strong boundaries. Nature communications 9, 542, doi:10.1038/s41467018-03017-1 (2018).

23 Whyte, W. A. et al. Master transcription factors and mediator establish super-enhancers at key cell identity genes. Cell 153, 307-319, doi:10.1016/j.cell.2013.03.035 (2013).

24 Ross-Innes, C. S. et al. Differential oestrogen receptor binding is associated with clinical outcome in breast cancer. Nature 481, 389-393, doi:10.1038/nature10730 (2012).

25 Wang, H. et al. NOTCH1-RBPJ complexes drive target gene expression through dynamic interactions with superenhancers. Proceedings of the National Academy of Sciences 111, 705-710, doi:10.1073/pnas.1315023111 (2014).

26 Sheffield, N. C. \& Bock, C. LOLA: enrichment analysis for genomic region sets and regulatory elements in R and Bioconductor. Bioinformatics 32, 587-589 (2015). 
27 Phanstiel, D. H., Boyle, A. P., Heidari, N. \& Snyder, M. P. Mango: a bias-correcting ChIA-PET analysis pipeline. Bioinformatics 31, 3092-3098, doi:10.1093/bioinformatics/btv336 (2015).

28 Chakraborty, A. \& Ay, F. Identification of copy number variations and translocations in cancer cells from Hi-C data. Bioinformatics, doi:10.1093/bioinformatics/btx664 (2017). 